\title{
Applied System Identification for a Four Wheel Reaction Wheel Platform
}

\author{
A Thesis \\ Presented to the Faculty of the \\ California Polytechnic State University, San Luis Obispo
}

In Partial Fulfillment of the Requirements

for the Degree of a Masters of Science

in Aerospace Engineering

By

Seth Franklyn Silva

December 2008 
(C) 2008

Seth Franklyn Silva

ALL RIGHTS RESERVED 


\section{APROVAL PAGE}

TITLE: $\quad$ Applied System Identification for a Four Wheel Reaction Wheel Platform

$\begin{array}{lr}\text { AUTHOR: } & \text { Seth F. Silva } \\ \text { DATE SUBMITTED: } & \text { December } 2008\end{array}$

Committee Chair and Advisor:

Committee Member:

Committee Member:

Committee Member:
Dr. Eric Mehiel

Dr. Jordi Puig-Suari

Dr. Kira Abercromby

Dr. Charles Birdsong 


\begin{abstract}
Applied System Identification for a Four Wheel Reaction Wheel Platform

By

Seth Franklyn Silva
\end{abstract}

At the California Polytechnic State University, San Luis Obispo there is a four-wheel reaction wheel pyramidal simulator platform supported by an air-bearing. This simulator has the current capability to measure the wheel speeds and angular velocity of the platform, and with these measurements, the system identification process was used to obtain the mass properties of this simulator. A handling algorithm was developed to allow wireless data acquisition and command to the spacecraft simulator from a "ground" computer allowing the simulator to be free of induced torques due to wiring. The system identification algorithm using a least squares estimation scheme was tested on this simulator and compared to theoretical analysis. The resultant principle inertia about the z-axis from the experimental analysis was 3.5 percent off the theoretical, while the other inertias had an error of up to 187 percent. The error is explained as noise attributed to noise in the measurement, averaging inconsistencies, low bandwidth, and derivation of accelerations from measured data. 


\section{ACKNOWLEDGEMENTS}

I would like to thank my thesis advisor, Dr. Eric Mehiel, for his guidance and his ability to get me to take control and really understand a problem and critically solve it, also for his patience and helpfulness whenever I would get stuck. I would also like to thank my committee members who were patient throughout my many reschedules for my defense, and for their support, time, and understanding.

Thank you to all of the Cal Poly, San Luis Obispo Aerospace Department that instilled into me the "Learn By Doing" philosophy. Also for the many late nights or never-ending nights that showed me the joy of truly trying my best to solve an engineering problem.

I especially want to thank the Newman Catholic Center of San Luis Obispo for their lifechanging support and guidance through some of the hardest years in my life. Without Newman, I don't know if I could have gotten to where I am today. I truly thank you Fr. John, Fr. Kevin, and Linda.

I would also like to especially thank my parents for their continuing support and patience through my whole life, especially my years at Cal Poly. To my father who showed me how to truly get up and keep going, and my mother for her inspiration to persevere and her unconditional love.

Finally, I would like to thank God, who has truly blessed me with this education that I received here at Cal Poly. 


\section{Table of Contents}

\section{APPLIED SYSTEM IDENTIFICATION FOR A FOUR WHEEL REACTION WHEEL

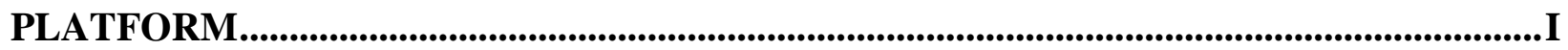

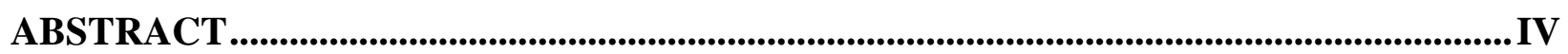

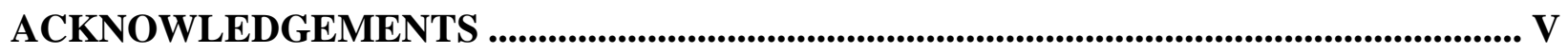

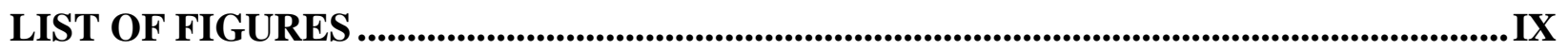

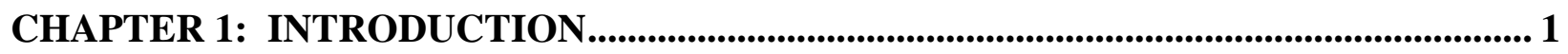

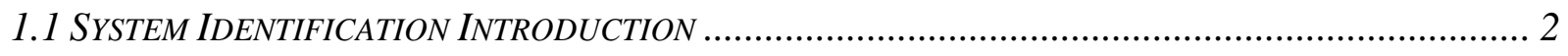

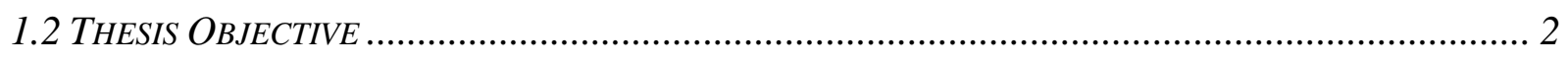

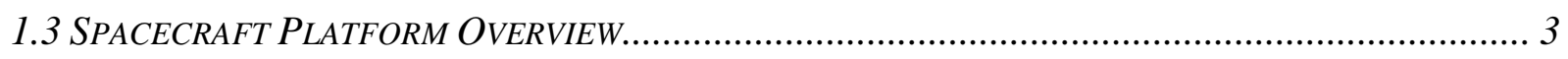

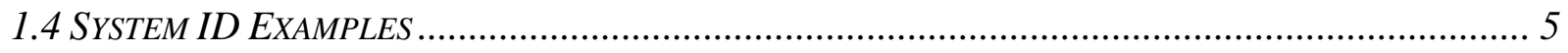

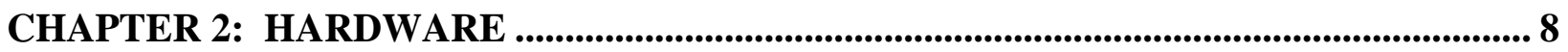

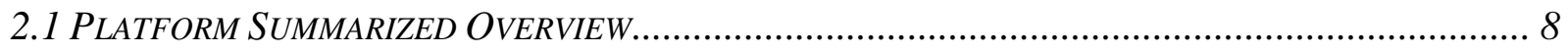

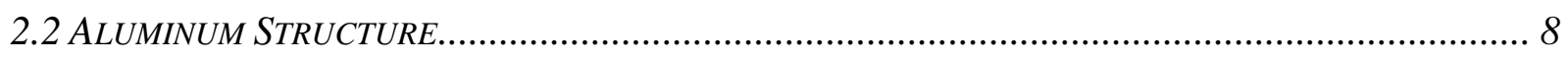

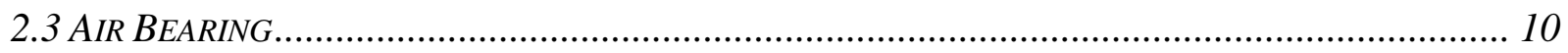

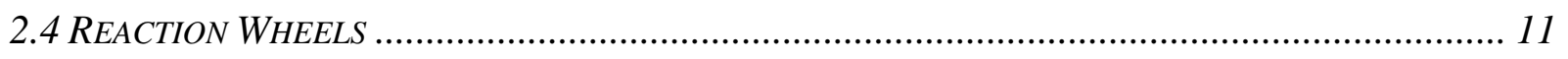

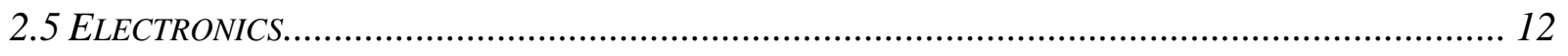

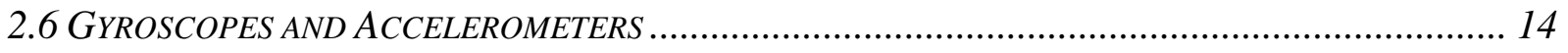

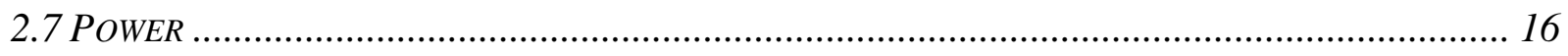

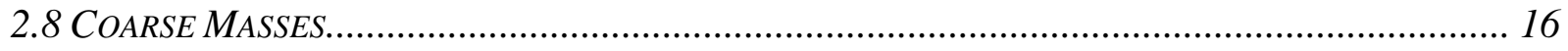

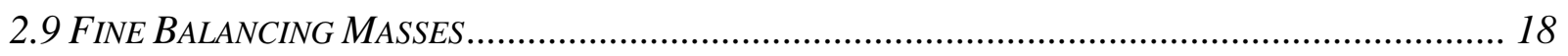




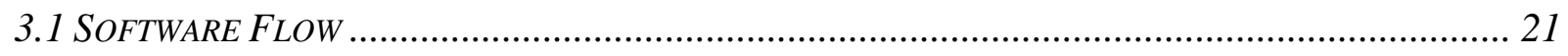

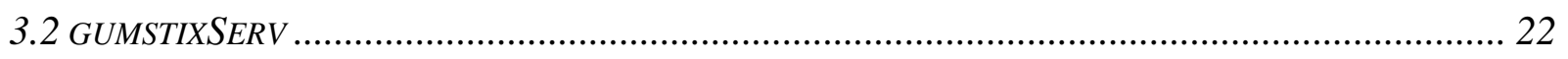

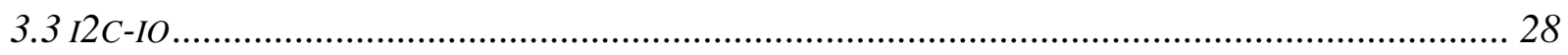

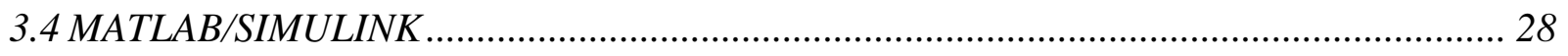

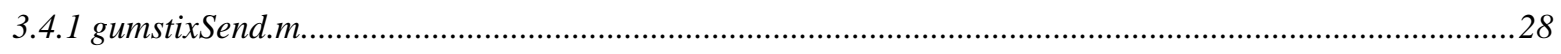

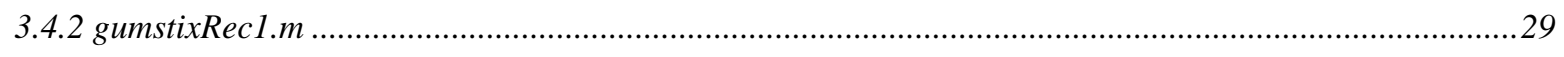

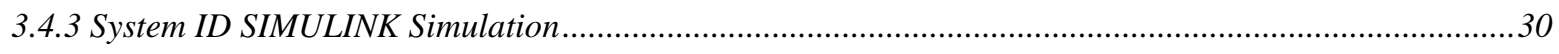

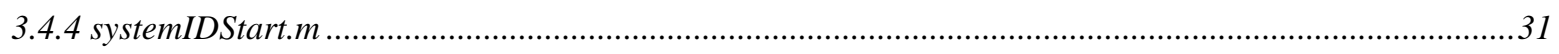

CHAPTER 4: THEORETICAL AND EXPERIMENTAL ANALYSIS .............................. 34

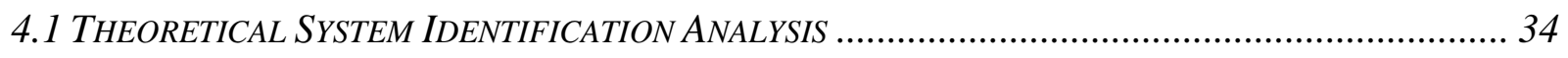

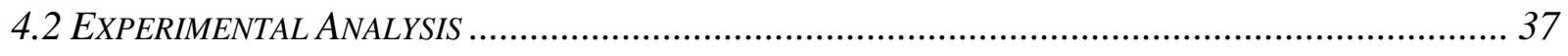

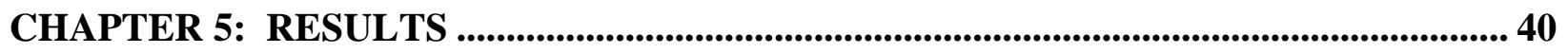

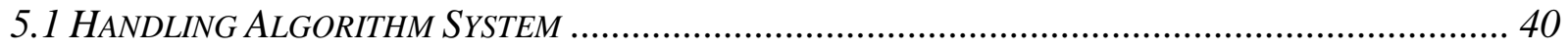

5.2 VERIFICATION OF EXPERIMENTAL SIMULATION PROCESS .................................................. 41

5.3 THEORETICAL AND EXPERIMENTAL ANALYSIS COMPARISON................................................. 43

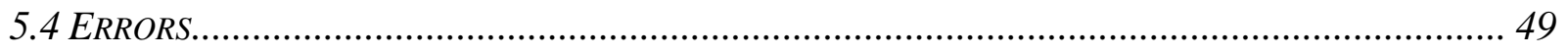

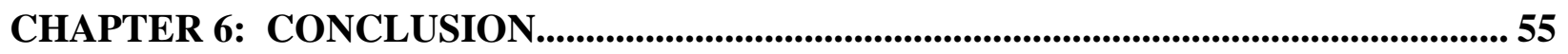

CHAPTER 7: FUTURE WORK AND RECOMMENDATIONS........................................... 57

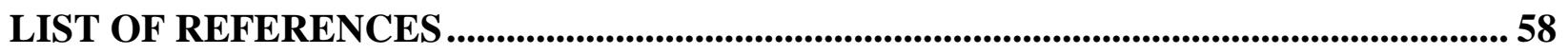

APPENDIX A: SIMULATION PROCEDURE ............................................................................ 60

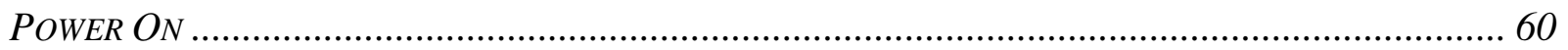




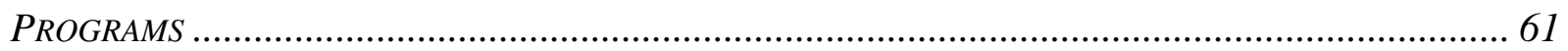

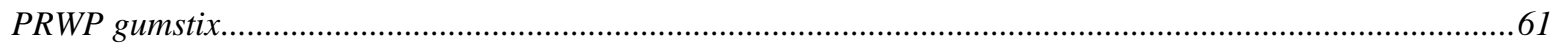

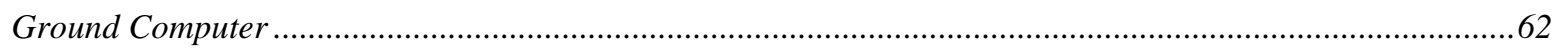

APPENDIX B: C AND MATLAB CODE AND SIMULINK MODEL FOR

EXPERIMENTAL SYSTEM IDENTIFICATION ..........................................................................63

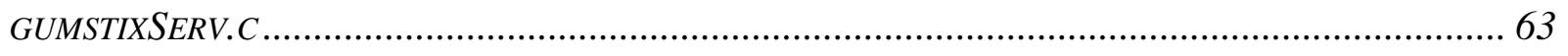

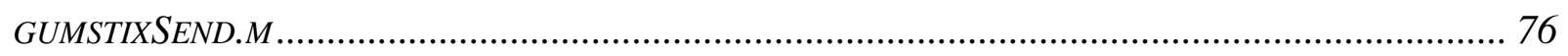

APPENDIX C: MATLAB CODE AND SIMULINK MODELS FOR POST-

PROCESSING ALGORITHM................................................................................................................. 96

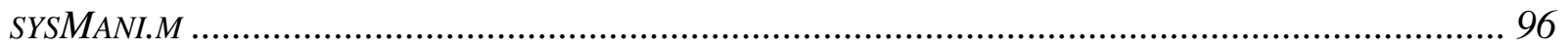

SYSTEMIDREAL_CHECK_V2 SIMULATION MODEL ..................................................... 102 


\section{List of Figures}

Figure 1: Cal Poly Pyramidal Reaction Wheel Platform ......................................................... 1

Figure 2: Mainboard with gumstix and robostix connected ............................................. 4

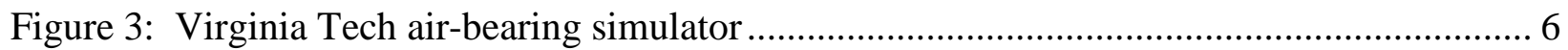

Figure 4: $\mathrm{x}$-axis coarse mass channel and counterweight ................................................ 9

Figure 5: $\mathrm{z}$-axis coarse mass channel and $\mathrm{x}$-axis coarse mass track ..................................... 10

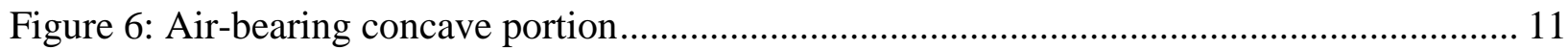

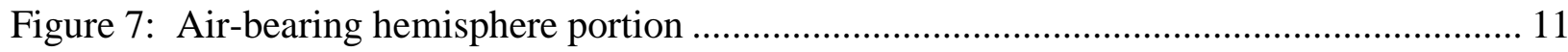

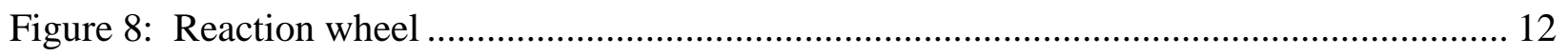

Figure 9: Gumstix microcomputer with Bluetooth dongle ................................................ 13

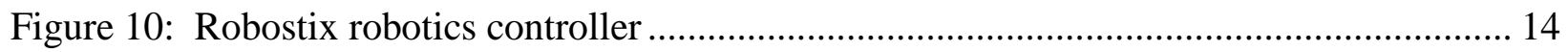

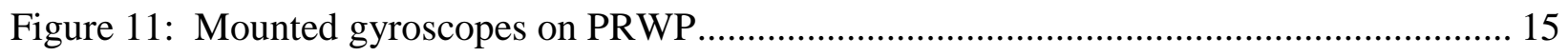

Figure 12: Main battery packs, 2 12-volt lead-acid batteries in series ................................... 16

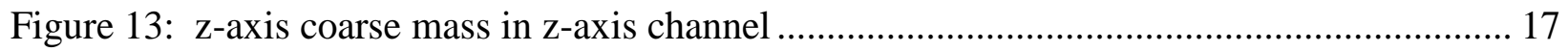

Figure 14: Center of mass fine balancing system for one axis ............................................. 18

Figure 15: $\mathrm{x}$-axis Center of Mass Fine Balancing System.................................................. 19

Figure 16: FAULHABER microcontroller for the CMFBS …........................................... 20

Figure 17: Software flow Diagram for Client/Server Communication .................................. 22

Figure 18: gumstixServ server software flow diagram ...................................................... 27

Figure 19: System ID Simulation Runtime Process .......................................................... 33

Figure 20: Theoretical identified state matrix compared to experimental identified state matrix

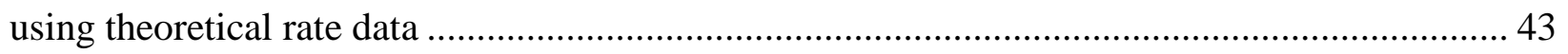

Figure 21: Comparison of theoretical and experimental identified state matrices .................... 44

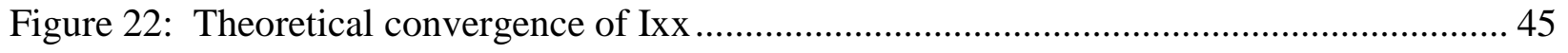

Figure 23: Experimental convergence of Ixx (top - full run; bottom - magnified).................... 45

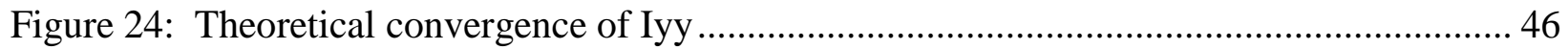

Figure 25: Experimental convergence of Iyy (top - full run; bottom - magnified).................... 46

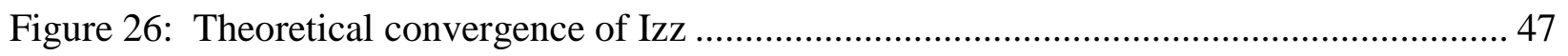

Figure 27: Experimental convergence of Izz (top - full run; bottom - magnified) .................... 48

Figure 28: Experimental raw angular velocity data ....................................................... 50 
Figure 29: Angular velocity (Red - raw data; Blue - averaged data) ..................................... 51

Figure 30: Identified state using averaged data vs. identified state without averaging .............. 52

Figure 31: Angular velocity raw versus averaged using bad averaging scheme ...................... 53

Figure 32: Theoretical high bandwidth versus low bandwidth identified matrices................... 54 


\section{Chapter 1: Introduction}

California Polytechnic State University, San Luis Obispo has a four wheel reaction wheel spacecraft simulator. This simulator is supported by an air bearing as well. There are four reaction wheels with one for redundancy, and the wheels are oriented in a pyramidal configuration with a 63 degree inclination angle. The pyramidal reaction wheel platform (PRWP) has gyroscopes to measure angular velocity and these velocities are used to get acceleration as well. System identification is used by this platform and then the identified mass properties of the simulator are used to move counterweights to attempt to balance the spacecraft simulator. The PRWP is shown below in Figure 1.

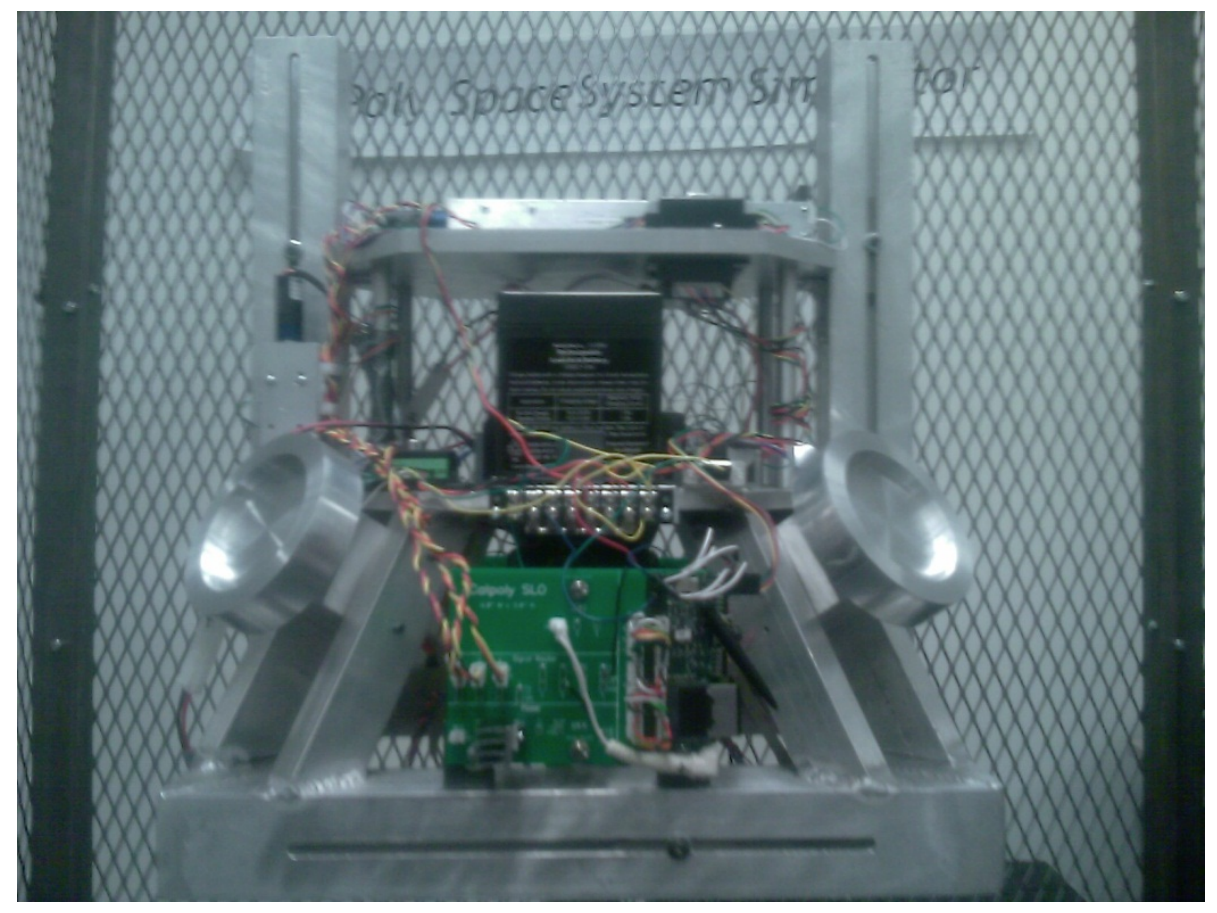

Figure 1: Cal Poly Pyramidal Reaction Wheel Platform 


\subsection{System Identification Introduction}

The main purpose of system identification is to identify the physical attributes of a system through experimental measurements and data. ${ }^{1}$ The process of system identification for this case, a spacecraft simulator, is to intentionally disturb the system with some sort of external torque. Then sensor data is taken in to characterize the effects of that external torque. Through initiating a disturbance and recording its effects, the physical system is approximately determined through extrapolating it from the system's equations of dynamic motion. Patrick Healy, a graduate student at Cal Poly, San Luis Obispo wrote a thesis on the theory behind system identification. $^{2}$ His thesis goes on to explain how the dynamic equations are manipulated to identify the system. The analysis section of this thesis describes Healy's thesis in more detail.

\subsection{Thesis Objective}

The first objective of this thesis is to design a handling algorithm system for communication between the onboard computer on the platform and the ground computer. One aspect of this handling system is a server algorithm that allows the ground connection to connect to the platform wirelessly. The server receives commands from the ground, process those commands and perform the necessary tasks, and send data back to the ground computer. The second aspect of this handling system is a client connection through MATLAB to interface a MATLAB simulation with the server on the gumstix.

The second objective of this thesis is to apply the theoretical analysis of the system identification process found in Healy's thesis to a fully functional 4 wheel reaction wheel spacecraft platform. This application will be able to verify the process described in Healy's

\footnotetext{
${ }^{1}$ Juang, Jer-Nan. Applied System Identification. Upper Saddle River: Prentice Hall PTR, 1993.

${ }^{2}$ Healy, Patrick B. "Mass Property System Identification of a Spacecraft Simulator." Thesis.
} 
thesis as a viable, practical method to effectively identify the physical attributes of a system, in this case, the inertia properties of the simulator and the location of the center of mass in relation to the pivot point of the simulator. This thesis goes one step further than Healy's thesis by incorporating the Center of Mass Fine Balancing System (CMFBS). This system uses the location of the center of mass derived from the System Identification process to move the fine balancing masses a certain distance to try to minimize the difference between the center of mass and the center of rotation. This allows the platform to be fully balanced and centered in relation to the air bearing thus optimizing the effect of the reaction wheels.

\subsection{Spacecraft Platform Overview}

California Polytechnic State University, San Luis Obispo (Cal Poly, SLO) has a 4 wheel reaction wheel platform spacecraft simulator. The 4 wheels are arranged in a pyramidal configuration with each wheel angled at a 63 degree, from horizontal, inclination angle. The platform is composed of an aluminum alloy and is machined and welded into a pyramidal shape. Gyroscopes are used to measure the tangential velocity of the platform, which is then transformed to angular velocity of the entire platform. Also the acceleration is determined by taking the discrete derivative of the angular velocity reading and then transforming it into an angular acceleration. The center of the platform has a hemispherical ball that is mounted to the underside of the platform. The ball fits into a concave hemisphere that has ports that allow high pressure air fill the space between the ball and the concave hemisphere thus creating an air bearing. This air bearing allows the platform to move without much friction thus better simulating a space environment.

The platform motors and sensors are controlled by an onboard computer, shown in Figure 2, which will be described later. The onboard computer is connected to a robotics 
microcontroller that controls the reaction wheels, gyroscopes and later integrated accelerometers, and tachometers for the reaction wheels. The onboard computer has a wireless Bluetooth module to allow for wireless communication between the onboard computer and the main ground computer that runs the simulation that controls the spacecraft simulator. The platform also utilizes onboard power to truly be independent of wires from the ground, reducing the external torques experienced by the platform that could be applied by the presence of off board wires.

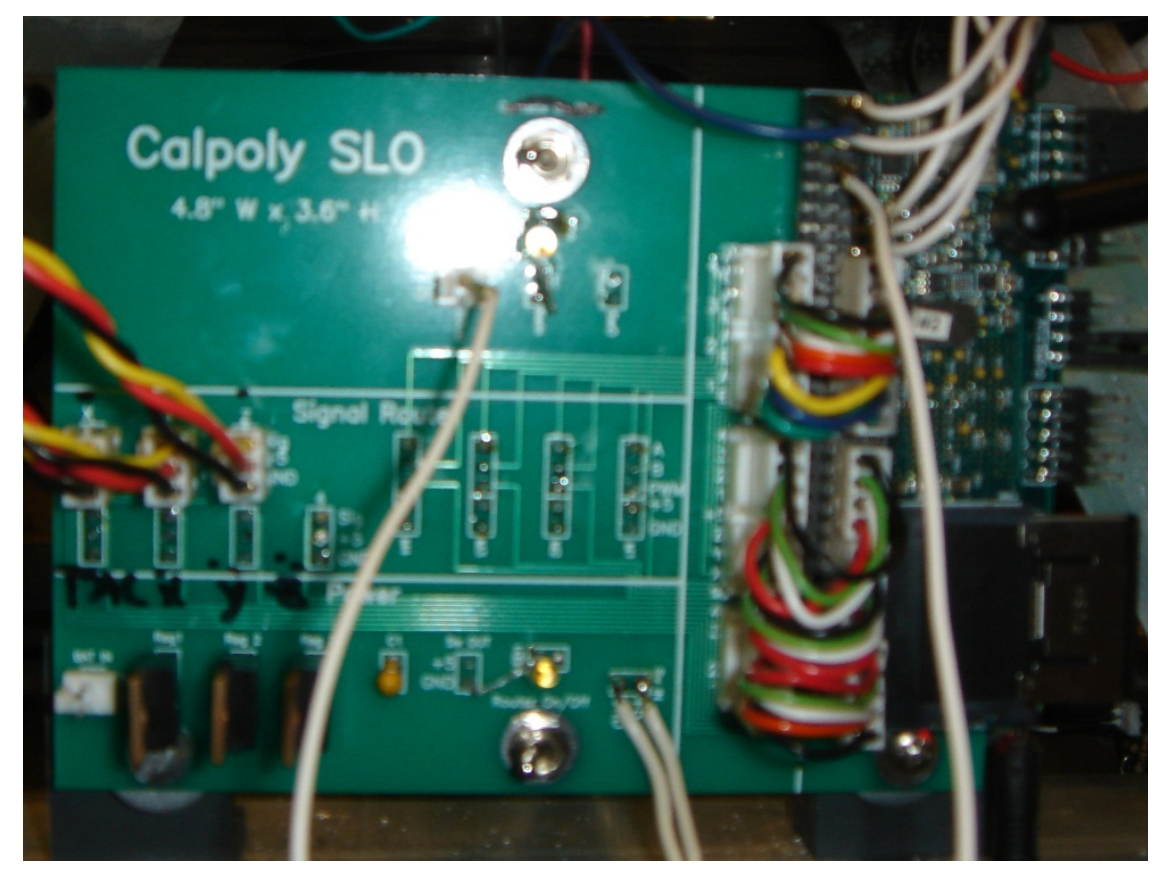

Figure 2: Mainboard with gumstix and robostix connected

Finally the platform's onboard computer is controlled through the Bluetooth connection by the ground computer, which is a Pentium 4 Dell computer. The ground computer runs MATLAB and SIMULINK to run the simulations necessary to command the reactions wheels at the appropriate rates to control the platform effectively. The SIMULINK program also reads in data from the sensors via the Bluetooth connection to monitor the effects of the platform due to the torques placed on it by the reaction wheels and gravity. This sending commands and reading 
data allows the control system to be a closed loop control system for the platform, which Jeff Logan goes into more detail in his thesis. ${ }^{3}$

\subsection{System ID Examples}

The control system for a space vehicle is tailored to the specific inertia properties of the craft, and when extra mass is placed inside the spacecraft offset from its center of mass or the mass properties might not be fully known, the control system itself is cannot be fully optimized due to the spacecraft's inertia properties being changed. One way to optimize the control system is to identify the new inertia properties of the spacecraft, and this is done through a method called system identification.

There are several different applications for where system identification can be utilized such as the identification of the system of an unmanned helicopter being used for crop dusting. System identification is used in this application by helping to determine the dynamics of the helicopter. While crop-dusting, there is a definite change in mass as the chemical being used to dust the field are being jettisoned. The computer for this unmanned helicopter measures the roll, pitch, yaw, and heaving of the helicopter. After this measurement, the dynamics of the helicopter can be identified through the measured output and the measurement of the input given through the actuators to control the helicopter. Once the mass properties of the helicopter are identified, those properties can allow the helicopter be better stabilized. ${ }^{4}$

Another example of system identification can be applied to a spacecraft simulator system such as the spacecraft simulation platform at Virginia Tech. Their platform consists of a 3-axis

\footnotetext{
${ }^{3}$ Logan, Jeff. Control of Pyramidal Reaction Wheel Platform. Thesis, 2008.

${ }^{4}$ Hashimot, Seiji, Tomonori Ogawa, Shuichi Adachi, Anzhong Tan, and Gou Miyamori. "System Identification Experiments on a Large-scale Unmanned Helicopter for Autonomous Flight." IEEE International Conference on Control Applications, 25-27 Sept. 2000, Anchorage, Alaska, USA.
} 
reaction wheel system, where the entire platform is elevated by a spherical air-bearing thus greatly reducing friction. The platform can be expanded to carry various amounts of payload up to 300 pounds. To help balance the system, the system identification process uses linear actuators to correct for the center of mass to be at the pivot point at the air-bearing. A figure of their platform is shown below in Figure 3.

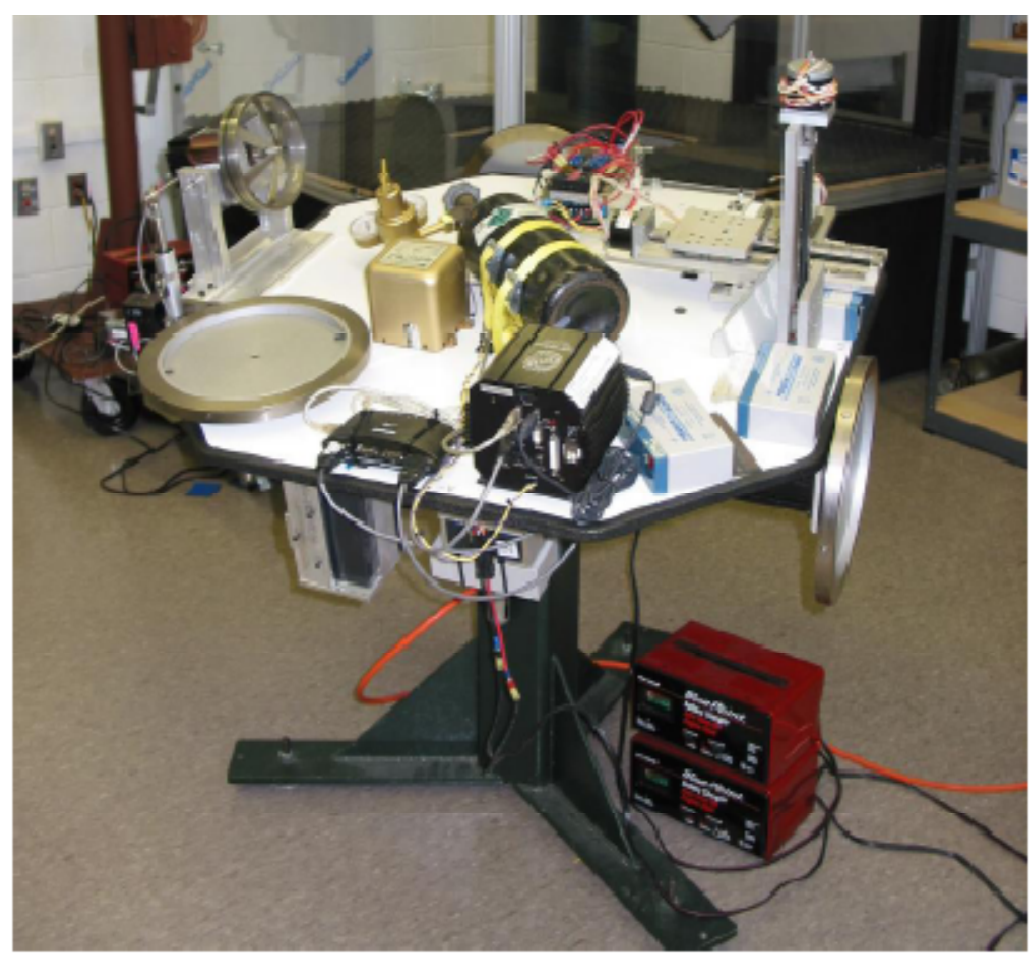

Figure 3: Virginia Tech air-bearing simulator

A second example of automatic mass balancing can be seen in the Naval Postgraduate School in Monterey, California. ${ }^{6}$ Kim and Agrawal describe a 3-axis stabilized spacecraft attitude simulator, located at the Naval Postgraduate School, containing an automatic balancing system to offset any center of gravity misalignments due to the hardware configuration of the platform. In order to truly balance the platform, Kim and Agrawal state that the platform must

\footnotetext{
${ }^{5}$ Schwartz, Jana L., and Christopher D. Hall. "Comparison of System Identification Techniques for a Spherical AirBearing Spacecraft Simulator." AAS/AIAA Astrodynamics Specialist Conference, 3-7 Aug. 2003.

${ }^{6}$ Kim, Jae Jun, and Brij N. Agrawal. "Automatic Mass Balancing of Air-Bearing-Based Three-Axis Rotational Spacecraft Simulator." JOURNAL OF GUIDANCE, CONTROL, AND DYNAMICS May-June 200932.3 (2009): 1005-017. Print.
} 
be excited to properly compensate the center of gravity offset. Through compensating the offset of the center of gravity they show that gravitational disturbances, resultant of the offset, can be reduced producing a much more exact simulation of space-bourne operation with minimum gravitational influences imposed on the simulation platform. In Figure 4, a photograph of the spacecraft simulator located at the Naval Postgraduate School is shown.

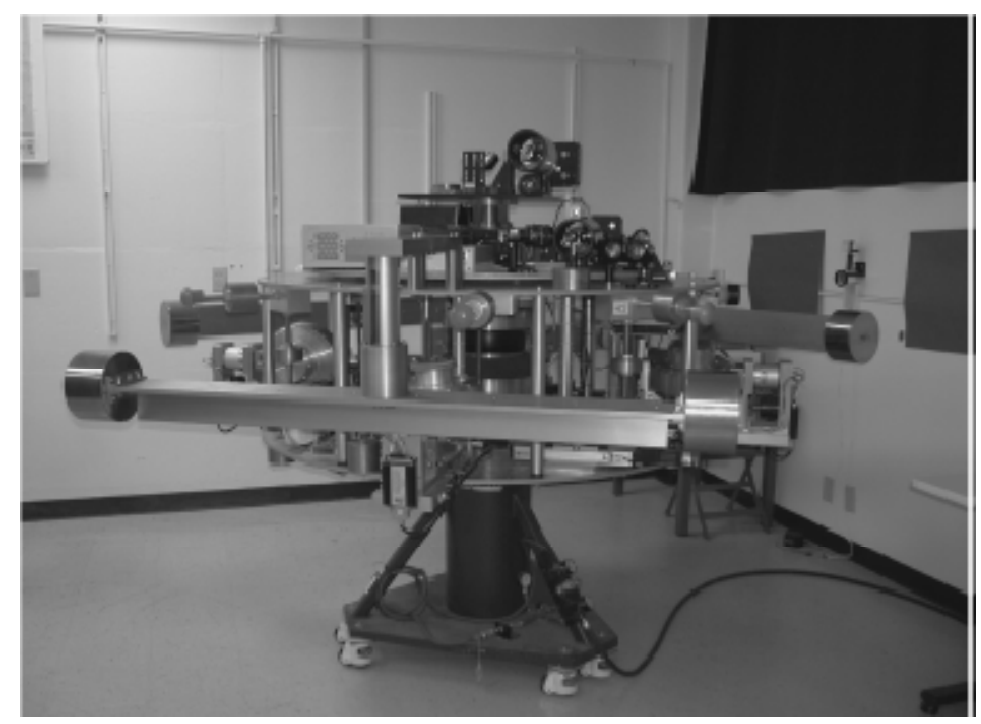

Figure 4: Naval Postgraduate School Spacecraft Simulator 


\section{Chapter 2: Hardware}

In order to test the system identification technique, a proper experimental apparatus is needed to accurately exercise the System ID process. The following chapter gives a summary of CalPoly's PRWP's hardware. The PRWP contains an aluminum structure, reaction wheel, and microcontroller.

\subsection{Platform Summarized Overview}

The Pyramidal Reaction Wheel Platform (PRWP) consists of high stiffness aluminum structure that is mounted on an air bearing. Aluminum reaction wheels provide attitude control through momentum exchange. An onboard computer and a microcontroller robotics driver send commands to the motor drivers and read in the sensor data. Sensors measure angular velocity and acceleration to monitor the effects of outside disturbance and/or reaction wheel torques on the system. There are manually adjustable coarse balance masses that can roughly balance the center of mass of the PRWP. In addition to the coarse masses, there are smaller masses connected to DC motors that can fine balance the PRWP. Finally there is a "ground" computer that runs the simulation that sends out desired reaction wheel outputs and takes the sensor data gathered by the PRWP on board computer to complete a closed loop control system. The ground computer communicates with the on board computer via a TCP/IP Bluetooth connection. The on board computer has an integrated Bluetooth module and the ground computer has a Bluetooth receiver.

\subsection{Aluminum Structure}

The PRWP stiff rigid aluminum structure is welded together to create a three tier configuration. The first tier, located at the bottom of the platform consists of four aluminum U- 
channels welded together to form a square. The u-channels each have a track cut into the sides of the channels to serve as a guide and a bolting point for 4 large masses, one for each side, that serve to coarsely balance the platform in the $\mathrm{x}$ and $\mathrm{y}$ directions. On top of the square of welded u-channels are aluminum bar stock welded at a 63 degree angle from horizontal. There are two pieces per corner and they hold the reaction wheel motors with the spin axis perpendicular to the 63 degree angle. On top of the angled pieces of aluminum is an aluminum shelf that contains the batteries and one fine balancing mass system for the y-direction. Above that shelf there is a smaller shelf mounted on round aluminum bar stock. This upper shelf contains the gyroscopes and another fine balancing mass system for the x-direction. Finally, mounted perpendicularly to the square u-channel structure and bracing the shelves of the platform are two u-channels, one on either side of the platform that contains a large mass for coarse mass balancing.

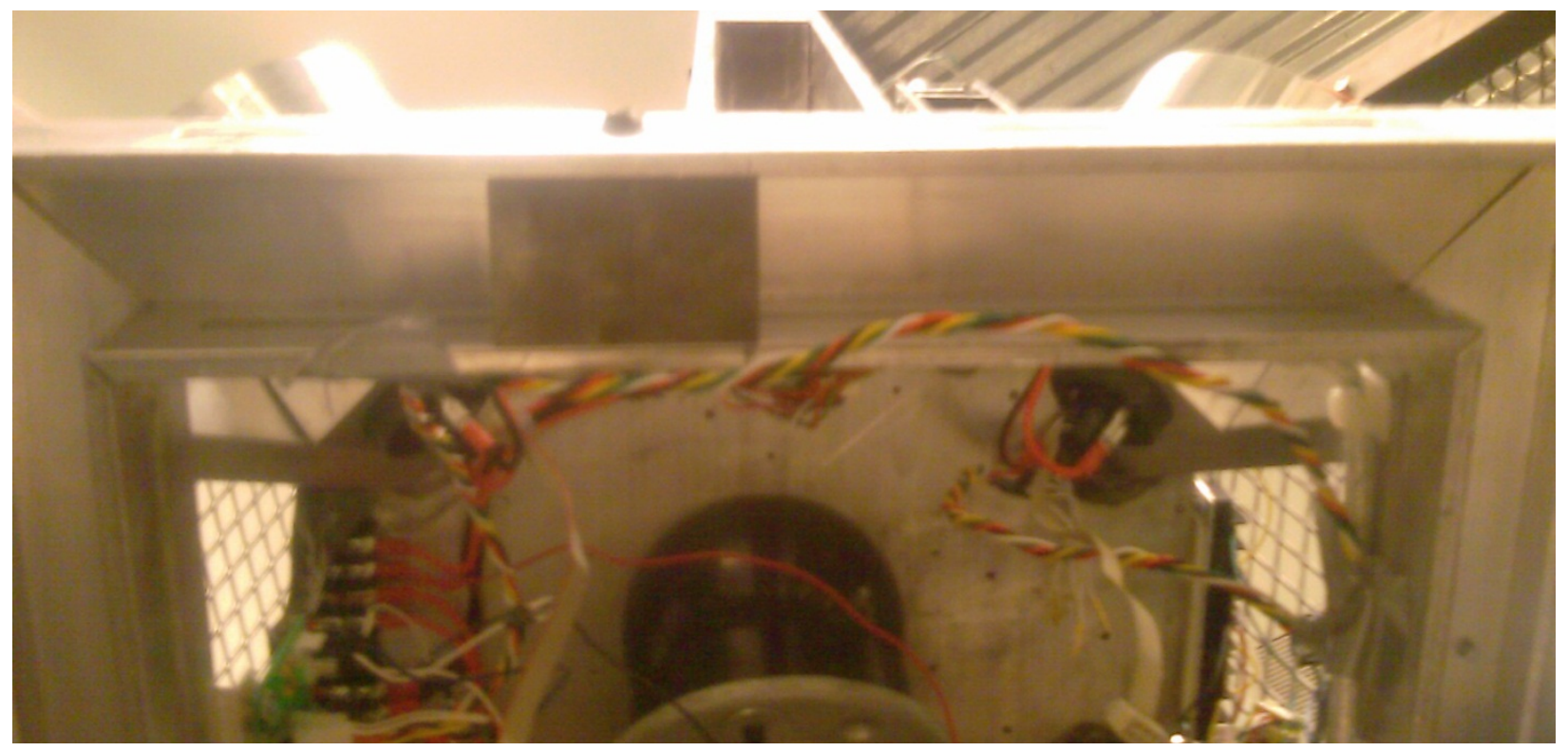

Figure 5: $x$-axis coarse mass channel and counterweight 


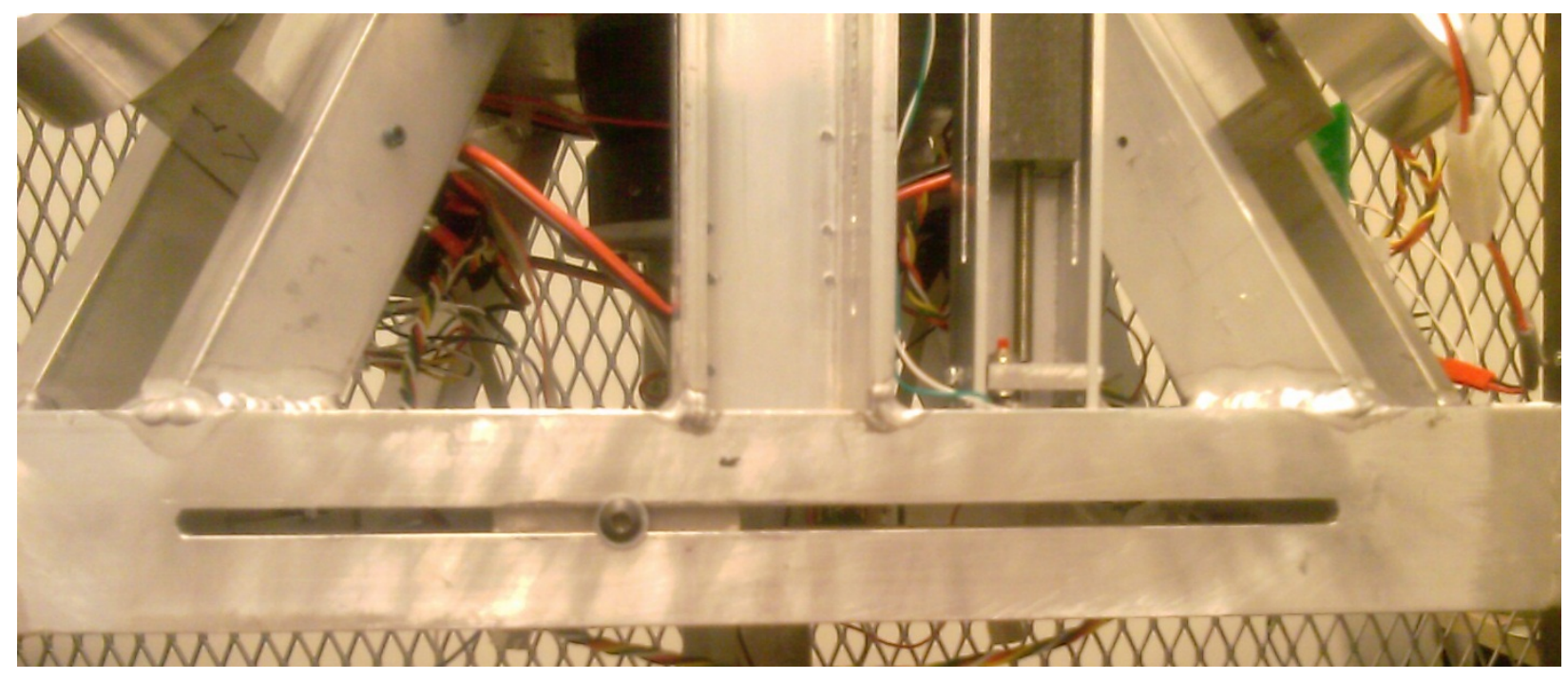

Figure 6: $z$-axis coarse mass channel and $x$-axis coarse mass track

\subsection{Air Bearing}

The PRWP is mounted on top of an air bearing consisting of a convex, hemispherical ball and a concave, hemispherical cup. Air, at an average pressure of 60 pounds per square inch, is blown through small air ports in the cup portion of the bearing creating an air buffer between the two parts of the air bearing. The pressure is produced by a compressor feeding the input port of the air bearing. The cup portion of the air bearing is mounted onto a circular metal pipe that is bolted to the frame of the safety cage housing the PRWP. The ball portion of the air bearing is centered and mounted to the bottom of the middle aluminum shelf. The location of the ball portion of the air bearing is the pivot point of the PRWP, which is also mentioned as the center of rotation $(\mathrm{CR})$ earlier in this paper. 


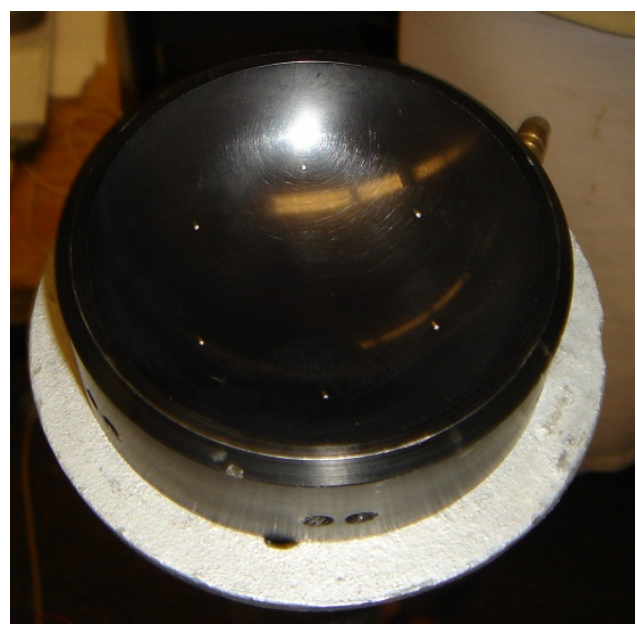

Figure 7: Air-bearing concave portion

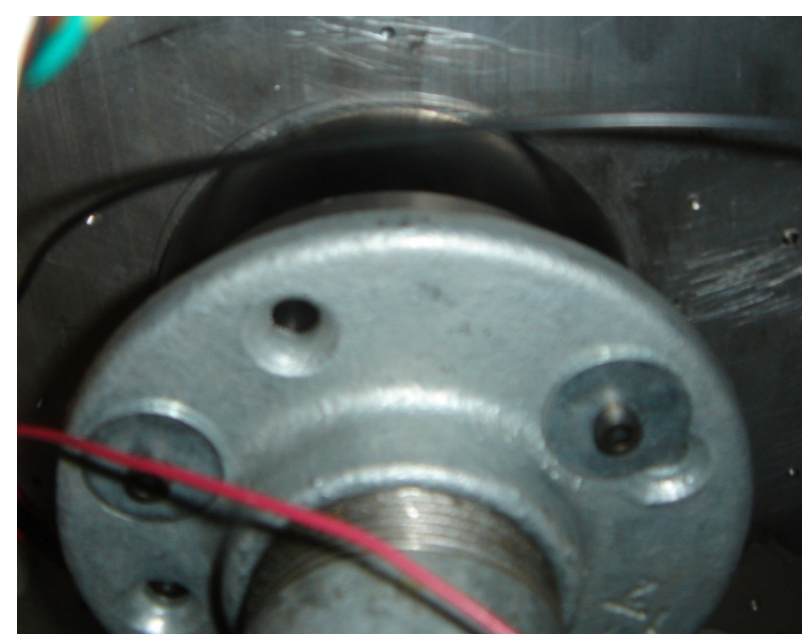

Figure 8: Air-bearing hemisphere portion

\subsection{Reaction Wheels}

The 4 reaction wheels that are apart of the platform are angled with the spin axis inclined by 57 degrees. The wheels are made of aluminum with inertias of $5.3552 \mathrm{~kg}-\mathrm{cm}^{2}{ }^{7}$ The wheels are powered by 4 FAULHABER DC micromotors each controlled by Polulu MD01B motor drivers. The wheels are commanded by pulse width modulation to determine the necessary speed for the wheels to spin. The pulse width signal is output by the microcontroller called a robostix that is then sent to the motor driver and then amplified and sent to the FAULHABER motor. The motors use a 24 volt system that is powered by an onboard battery.

\footnotetext{
${ }^{7}$ Carson, Mittelsteadt O. "Results on the Development of a Four-Wheel Pyramidal Reaction Wheel Platform." Thesis.
} 


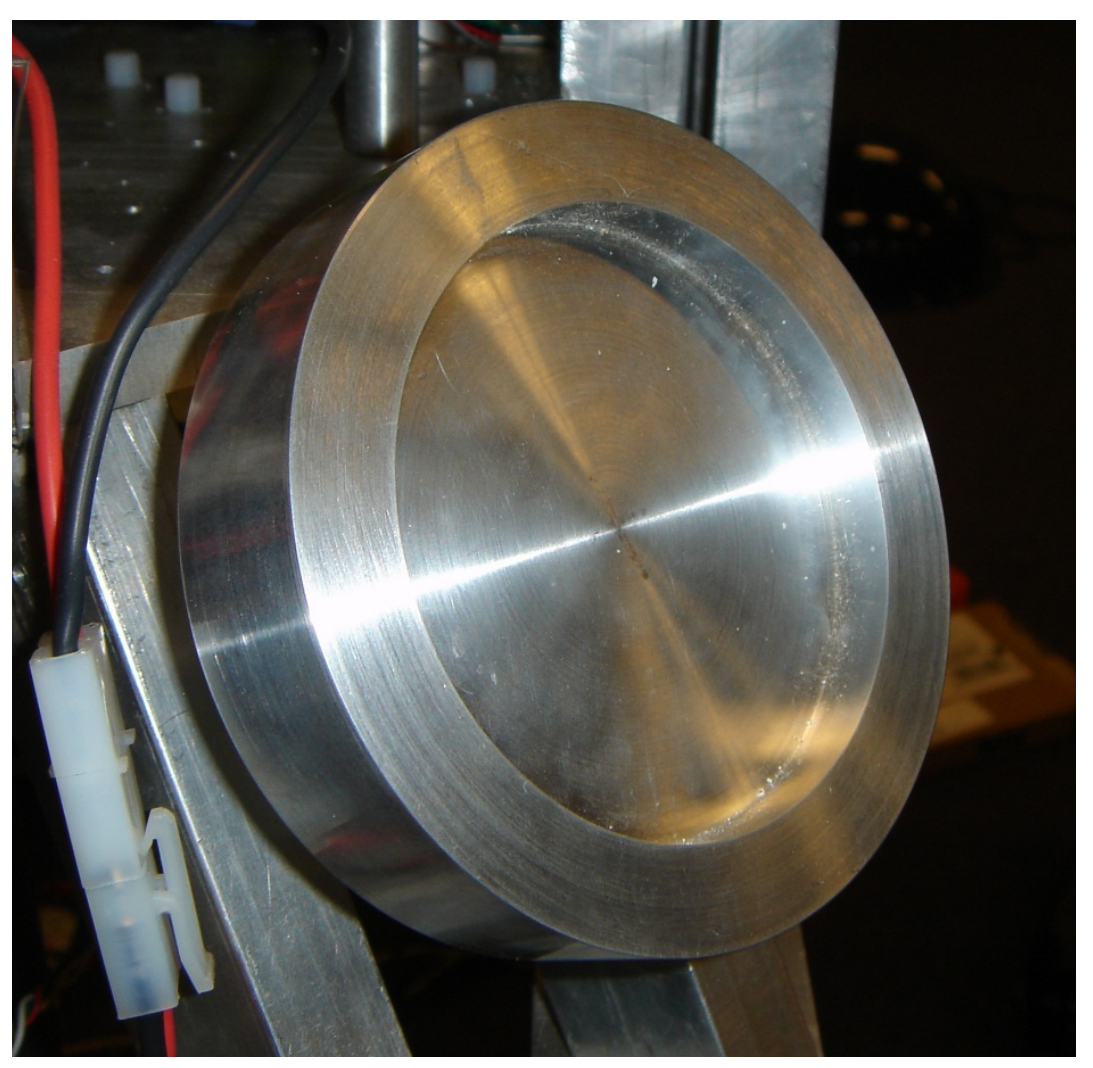

Figure 9: Reaction wheel

\subsection{Electronics}

The electronics consist of several different electronics boards. The main processor of the PRWP that sends and receives data and communicates with the ground computer is called the gumstix. Gumstix is a motherboard with a $400 \mathrm{MHz}$ Intel XScale PXA255 processor running Linux. ${ }^{8}$ The board has 64 MB RAM with a 16 MB flash memory and an MMC card adapter. The onboard memory allows for programs to be stored on board and then run. Also the MMC card allows the gumstix to expand in memory. Currently on the gumstix, there is a 1 gigabyte card allowing the storage of ample amounts of files and programs. Also on board the motherboard is a Bluetooth module allowing for wireless connectivity with the ground computer.

\footnotetext{
8 "Gumstix." Gumstix: Dream, Design, Deliver. Ed. Gumstix Inc. Gumstix. Web. <www.gumstix.com>.
} 
The gumstix also has a 60 pin Hirose input/output connector that allows the gumstix to be stacked on top of other expansion boards.

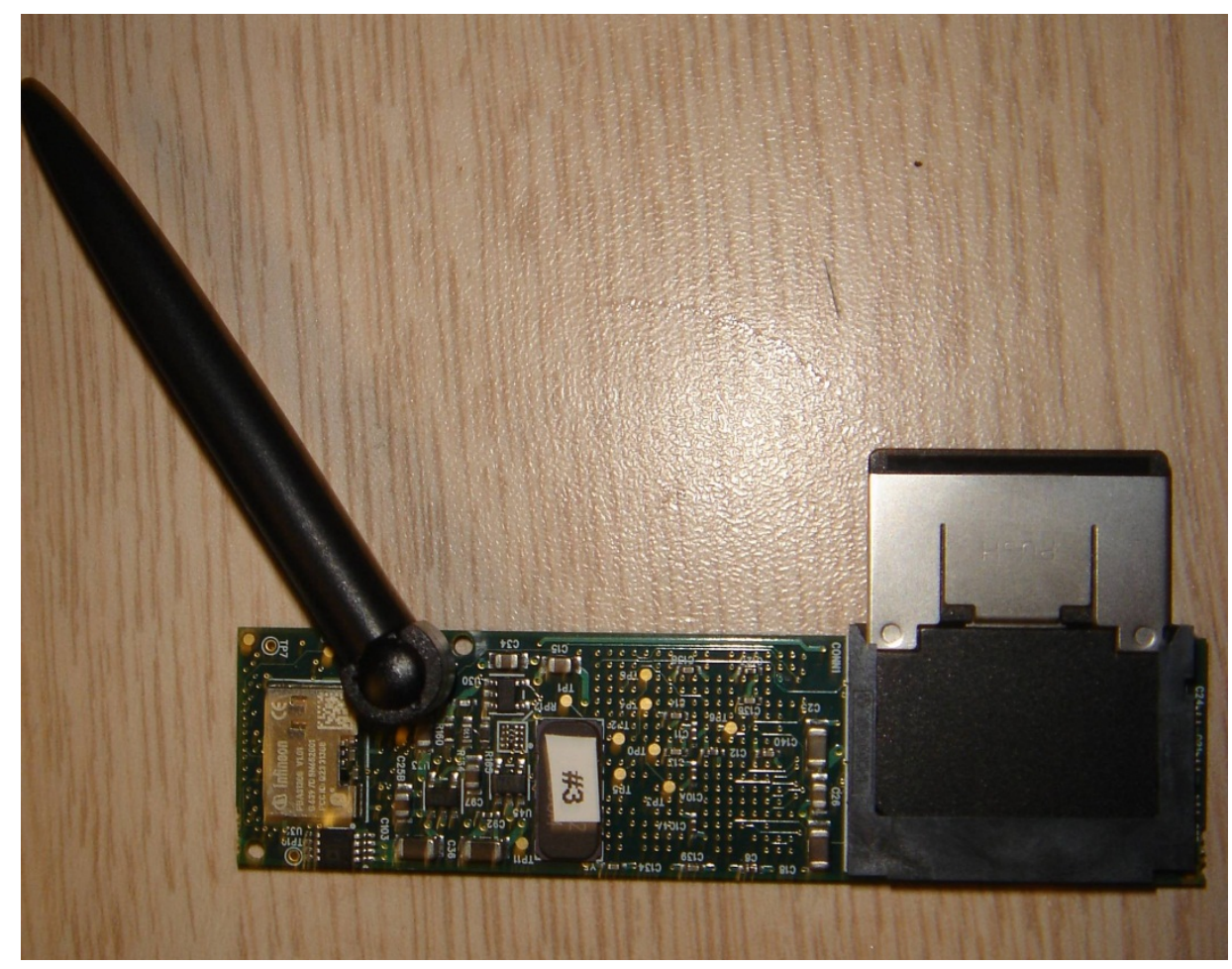

Figure 10: Gumstix microcomputer with Bluetooth dongle

The gumstix is connected, by the Hirose connector, to an expansion board called the robostix. The robostix is a robotic microcontroller with an ATMEL ATmega128 8-bit AVR microprocessor. The board contains a 60 pin Hirose connector that allows the gumstix to connect to the robostix and power the gumstix. The robostix contain eight pins connected to an analog to digital converter, six pulse-width modulator pins, sixteen general purpose input/output pins, and four serial port connections with one native to the gumstix kernel. A five volt signal can be taken from anyone of the middle row of pins, which allow the robostix to be able to power any peripheral device that might need to be connected. 


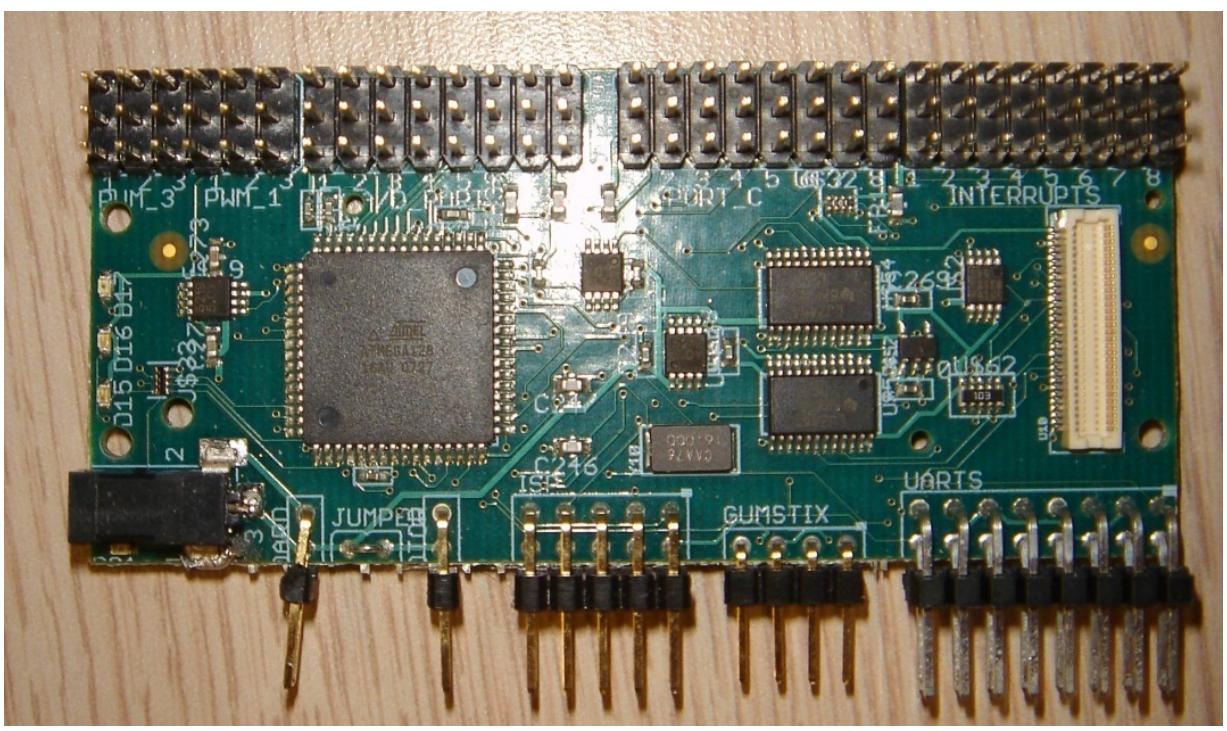

Figure 11: Robostix robotics controller

The final aspects of the electronics on the PRWP are the custom boards that were built for the platform by Jeff Logan. The mainboard that houses the gumstix and robostix combo is custom designed by Logan. The board consists of several power regulators to bring down the input voltage to the five volts that is necessary to power the gumstix/robostix. Also, the gyroscopes and tachometers are connected to this board and then routed to the robostix to be received and converted to digital inputs. The tachometers are also custom designed by Logan to output the actual speed of the reaction wheels. This allows the control system and the System Identification algorithms to more accurately approximate the actual speed of the wheels. More information on the boards can be found in Logan's thesis. ${ }^{9}$

\subsection{Gyroscopes and Accelerometers}

The sensors that measure the angular velocity of the PRWP are angular rate gyroscopes manufactured by Silicon Sensing. They have a range of +/- 100 degrees per second. The gyroscopes measure tangential velocity so the readings are transformed through SIMULINK to

\footnotetext{
${ }^{9}$ Logan, Jeff.
} 
get an angular velocity. There are three gyroscopes located on the platform and all three of them are located on the top shelf of the PRWP. They are mounted in line with the axis they are measuring, and they output $20 \mathrm{mV}$ per degree per second that they sense. The gyroscopes connect to the main electronics board that holds the robostix microcontroller and the onboard computer and their output is converted from an analog voltage to a digital reading to be sent over the Bluetooth connection to the ground computer. A 3-axis accelerometer manufactured by Sparkfun was originally integrated onto the PRWP, but their voltage range was found to be not large enough to record the accelerations that are experienced by the PRWP. Due to this shortcoming, the accelerations in the $\mathrm{x}, \mathrm{y}$, and $\mathrm{z}$ directions are extrapolated from the angular velocity readings from the gyroscopes by taking a discrete derivative of the angular velocities. There is some error introduced to the system, but that error is assumed negligible.

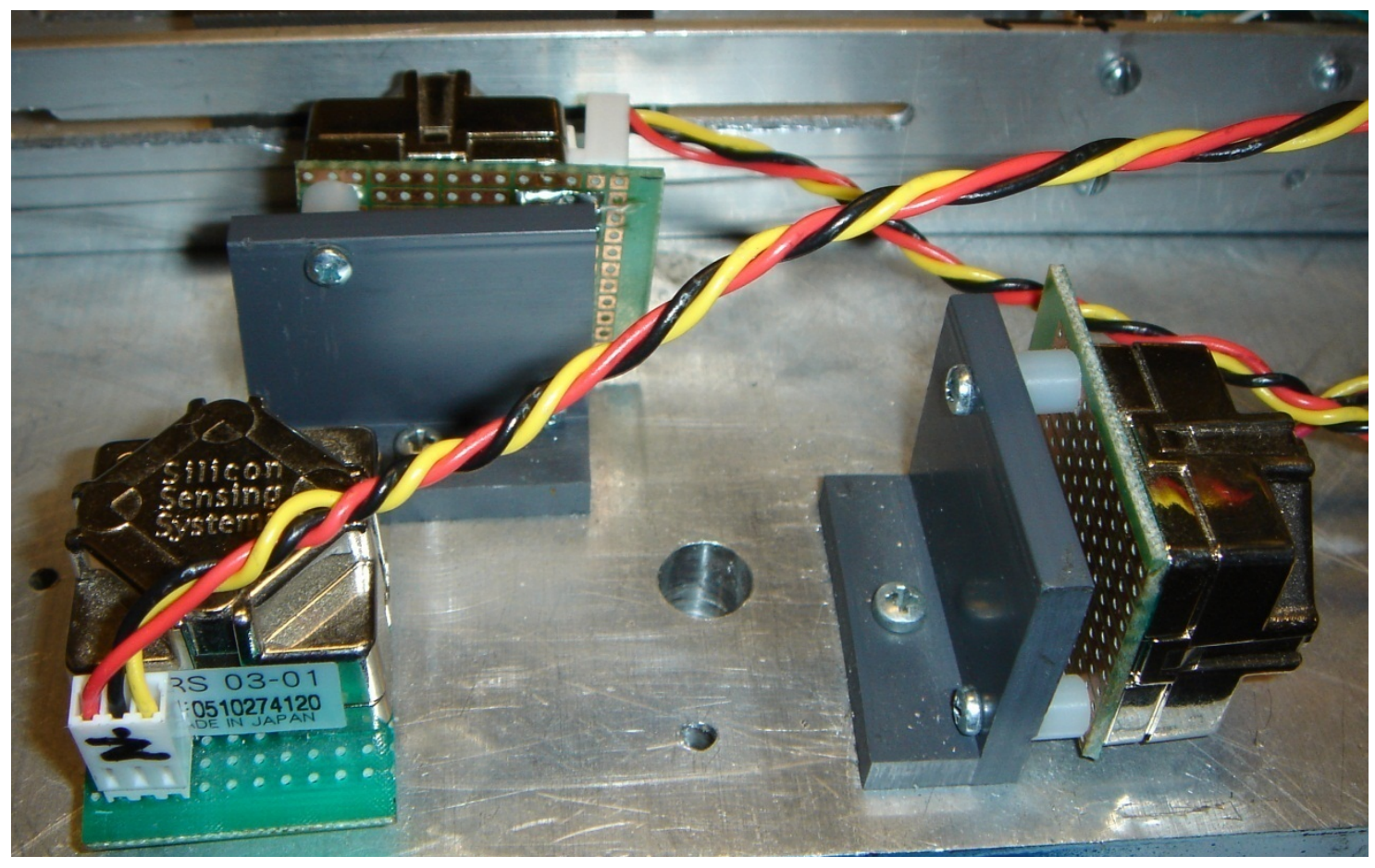

Figure 12: Mounted gyroscopes on PRWP 


\subsection{Power}

The power supplied to the PRWP is taken from an on board power supply. Two 12 volt lead-acid rechargeable batteries connected in serial create a 24 -Volt power supply necessary to power the reaction wheels and the microcontrollers controlling the fine balancing system motors. The power is routed using two terminal strips. One terminal strip routes the 24 volts of power to each reaction wheel driver and also to the second terminal strip. The second terminal strip carries the 24 volt power to three microcontrollers that control the fine balancing mass motors. The terminal strip also connects the serial port from the gumstix and daisy-chains the signals to the three microcontrollers. This allows the gumstix to control all three masses with one serial connection, which is described in more detail later in the paper.

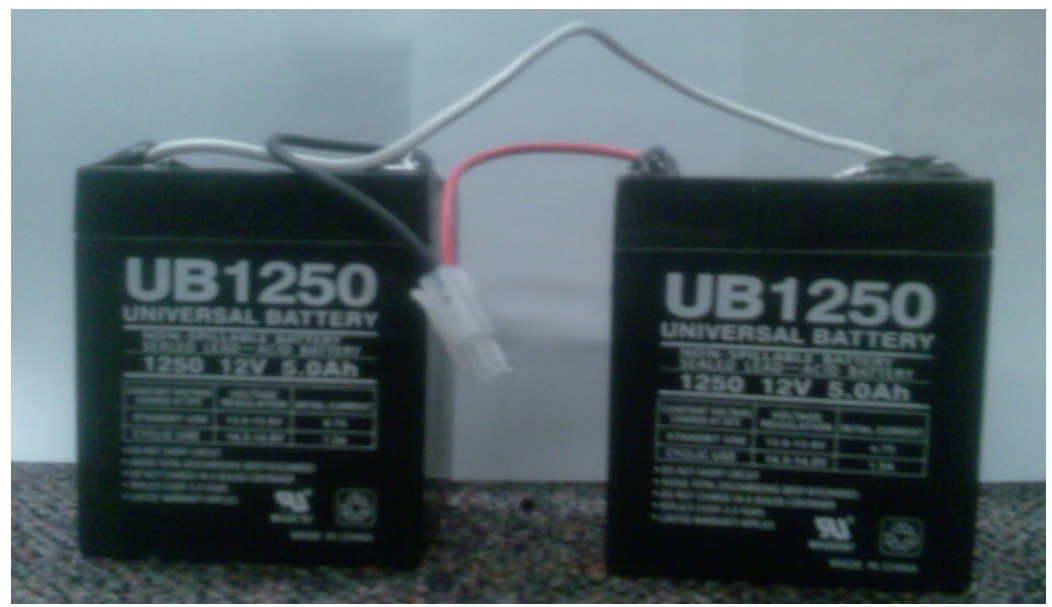

Figure 13: Main battery packs, 2 12-volt lead-acid batteries in series

\subsection{Coarse Masses}

There are six coarse masses, counterweights, located on the PRWP used to initially balance the platform. There are two masses per $\mathrm{x}, \mathrm{y}$, and $\mathrm{z}$ axes; four are located at the base of the platform to adjust the $\mathrm{x}$ and $\mathrm{y}$ axes while two more counterweights are mounted on two vertical u-channels on either side of the PRWP to adjust the z-axis. These masses, counterweights, are found in large u-channels with tracks cut into the sides of the channels to 
guide the weights and allow them to be bolted in place by a bolt with washers on each end that go through the mass and the tracks. Each weight is made of steel with an approximate mass of 0.77 kilograms. ${ }^{10}$

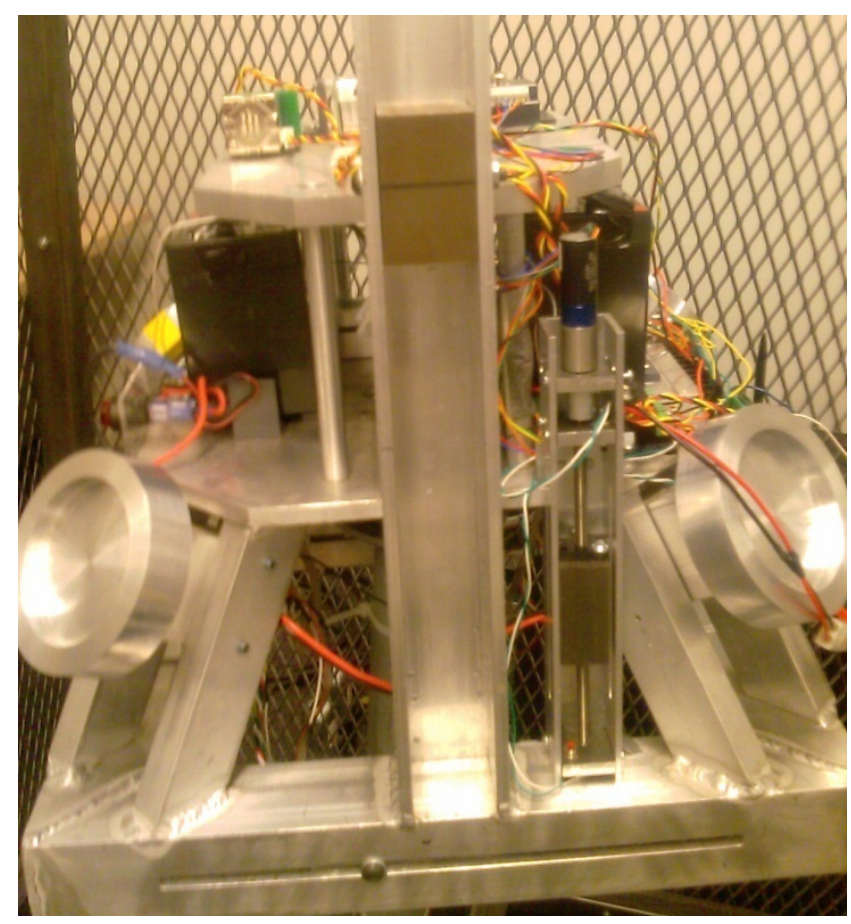

Figure 14: $\mathrm{z}$-axis coarse mass in z-axis channel

\footnotetext{
${ }^{10}$ Saile, Christopher A. Center of Mass Fine Tuning System for the Cal Poly Spacecraft Simulator.
} 

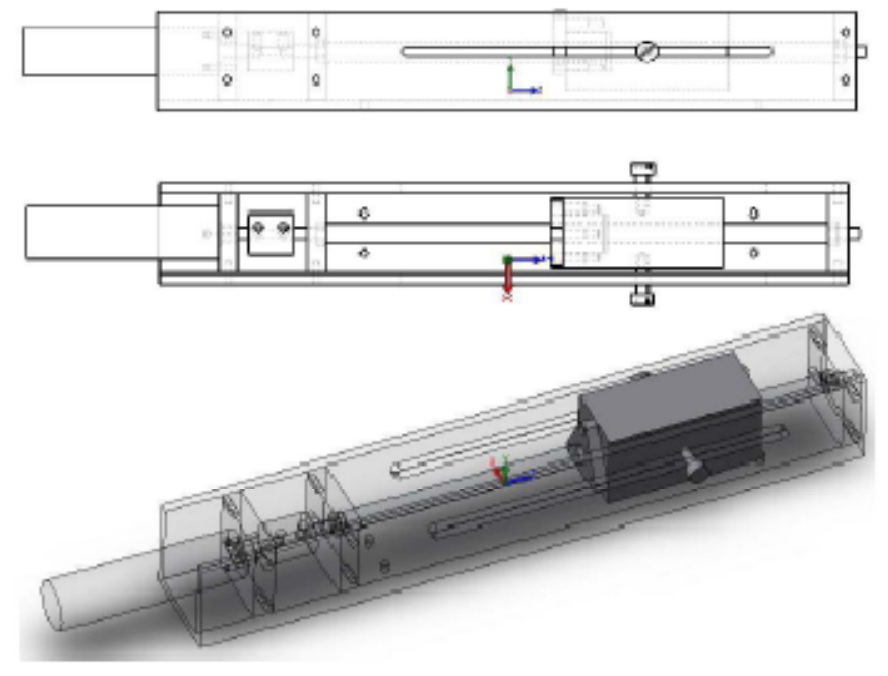

Figure 15: Center of mass fine balancing system for one axis

The center of mass fine balancing system (CMFBS) is used to more accurately balance

the PRWP after the coarse masses have initially balanced the platform. There are three fine balancing mechanisms, one for each axis, and a solid model of this mechanism is seen in Figure 15. The counterweights are designed to affect the axis with which its motion is aligned. The fine balancing counterweights are mounted in small aluminum u-channels with metal pegs passing through a slot machined in the u-channel and the counterweights itself, which allow the counterweights to move in a linear fashion. Each counterweight is made of steel bar stock that is cut to weigh 0.195 kilograms. The counterweights are designed to move the center of mass 0.08 centimeters for the length of each track. The counterweights are moved in the u-channel by a screw and nut system. The XCM 1800 Anti-Backlash Supernut is mounted on each counterweight and that nut is connected to a lead screw. The lead screw that is used is an XCM_6X1 lead screw with a 7/16 inch diameter and 20 threads per inch. The lead screw is then connected to a FAULHABER 1628 series motor by an aluminum grommet with two set screws that fasten the lead screw with the motor shaft. The FAULHABER motor has a 16/7 series 
gearhead that has a $246: 1$ reduction ratio, which means that for every 246 revolutions of the motor, the gearhead outputs 1 revolution to the lead screw.

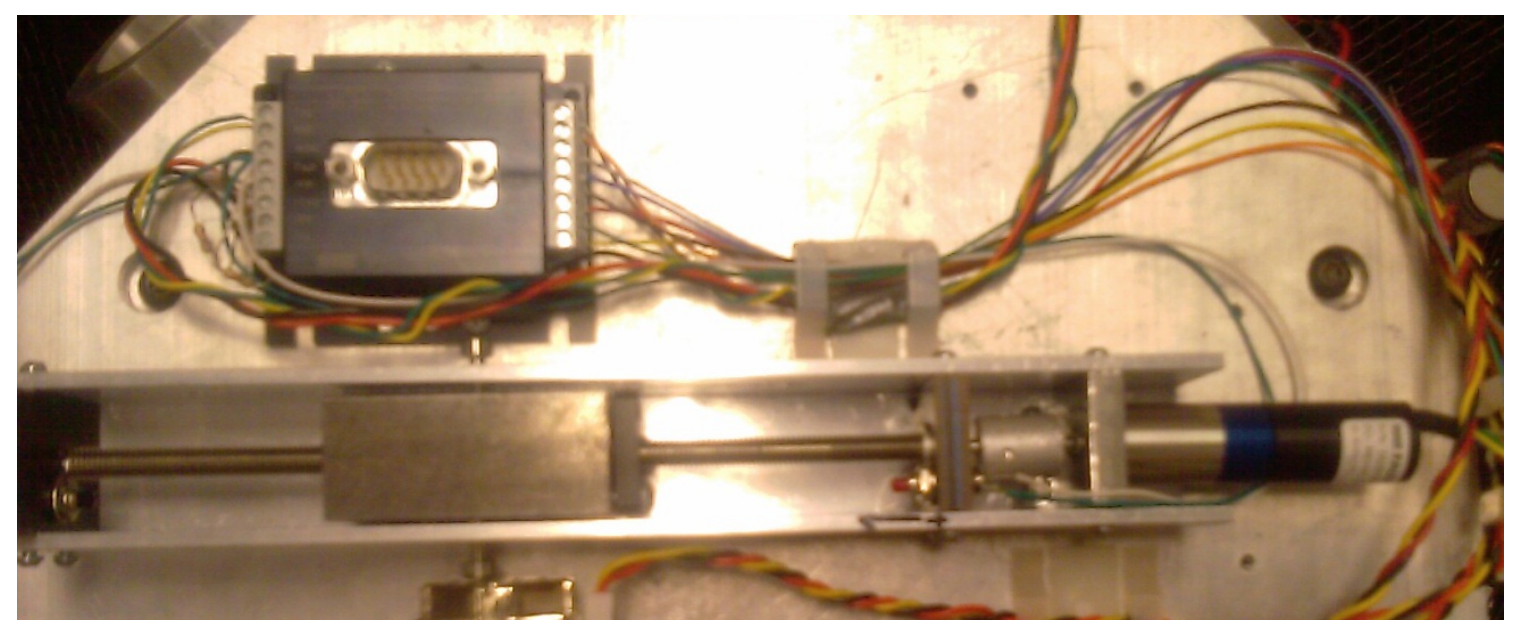

Figure 16: x-axis Center of Mass Fine Balancing System

The FAULHABER motors are controlled by a FAULHABER MCBL 2805 or MCBL 3006S microcontroller. These microcontrollers have the ability to command the velocity, position, or a special positioning sequence. They are communicated to by RS-232 serial communication protocol. For a positioning sequence, commanding the motor with a value of 1000 outputs one motor revolution, but with the 246:1 gearhead, a positioning value of 246000 will output a one true revolution to the lead screw. The FAULHABER microcontroller also has the ability to integrate switches to halt the motor when a switch is activated. Two momentary single-pole, single-throw switches, from Radio Shack, are mounted at either extreme of the fine balancing channels. When the counterweights are moved to either extreme of its track, the counterweights push against the switch triggering the switch and sending a signal to the microcontroller to halt the motor. This is useful to prevent the counterweights from moving past the boundaries of the fine balancing system tracks and prevent strain on the motors. These switches are connected to the analog and fault pin inputs on the microcontroller. 


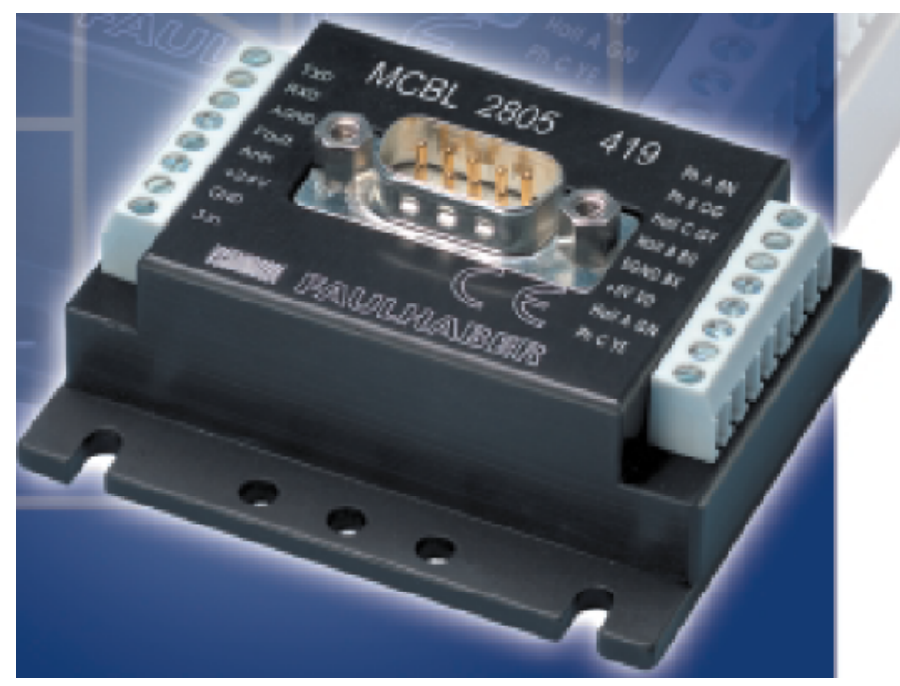

Figure 17: FAULHABER microcontroller for the CMFBS 


\section{Chapter 3: Software}

The first objective of this thesis is to develop a handling system for communication between the ground computer and the onboard computer, in this case, the gumstix Linux computer. The following chapter will explain the software flow of the server that will be implemented and the client protocol for commands to be sent and data to be received.

\subsection{Software Flow}

The gumstix acts as a server for the PRWP allowing MATLAB to connect to the gumstix as a client. MATLAB will connect and disconnect whenever commands need to be sent or data received by the simulation. The server and client use TCP/IP protocol through the wireless Bluetooth connection. The server configuration for the gumstix is optimal because the version of MATLAB that is installed on the ground computer does not have server capabilities. Also with the gumstix acting as a server, it allows the PRWP to always be listening for a client connection. The gumstix do not have to run any other program besides the server program allowing the ground computer to have more resources available to run MATLAB/SIMULINK. The gumstix will interface with the robostix through a communication protocol program called i2c. The server program and $\mathrm{i} 2 \mathrm{c}$ is explained later in this paper.

MATLAB/SIMULINK, as written before, acts as a client to the server running on the gumstix. MATLAB's SIMULINK program runs a block diagram simulation that evaluates and analyze the system and send commands to the gumstix depending on the analysis performed by the simulation. SIMULINK sends commands to the reaction wheels to initiate torque on the PRWP. Then SIMULINK reads in the angular velocity and acceleration of the system. Also the 
true wheel speeds are also read from the tachometers. A visual depiction of the software flow for this project is seen below in Figure 18 .

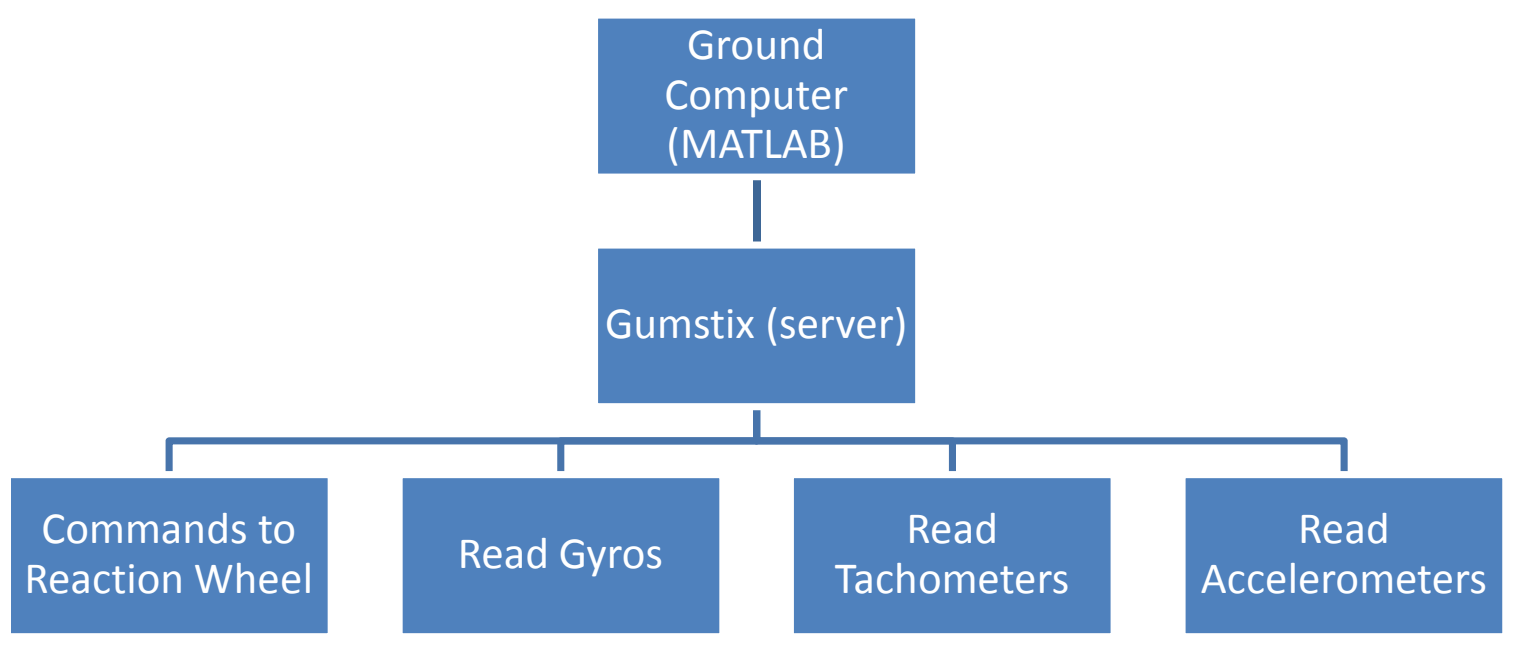

Figure 18: Software flow Diagram for Client/Server Communication

\section{2 gumstixServ}

The first program to be discussed is the server program that runs on the gumstix. A software flow diagram for this server is shown in Figure 19. This code was custom compiled for this application. When the server program is run, it first initializes the pins that will be used to read and write on the robostix through system calls to the i2c program interfacing the gumstix and robostix.

Once the pins are initialized, gumstixServ queries the user to either center the fine balancing system counterweights or skip that step. If the user chooses to center the counterweights, the server program sends a command to the FAULHABER microcontrollers to initiate a homing sequence that moves each counter weight in the CMFBS to one extreme of the track and then stop. Once the counterweights are moved to their extremes, the user selects the number one and the presses enter, and the FAULHABER microcontrollers are sent a command 
to move each counterweight to the center of their track. The format of these commands sent from the gumstix to the microcontrollers consists of one long string that holds commands for each of the FAULHABER controllers in one string. The string consists of new line characters (In) at the end of each command which delimit each command from the previous command. Also the number 0,1 , or 2 precedes each command to correspond to the node address of an individual FAULHABER microcontroller. These node addresses are preconfigured and saved into the microcontrollers on board memory. Once the masses are centered or if the user decides to skip the centering counterweight portion of the server program, the program then continues.

Then gumstixServ opens a listening TCP/IP socket used for connections with MATLAB. The listening socket is then bound and set to accept any IP address of any computer trying to connect with the port number of 8 . The socket is then set into listening mode. Once MATLAB sends a request to connect, the server accepts the connection and creates a connection client socket that contains the information for the client connection information, basically a file descriptor.

The gumstixServ program then listens for a command in the format of a string. The string is stored into a receive buffer and the first word is then parsed out. The first word in the string indicates the function that the gumstix/robostix combination is called to perform.

With the command indicator, gumstixServ program then goes to the appropriate part of an if-statement to carry out the command. For a reaction wheel command, a motor direction command and then a frequency and duty cycle command has to be given in order to command the motors the appropriate direction and speed for each wheel.

The "rwcomm" string is the frequency and duty cycle command. The receive buffer, after the "rwcomm" string contains a number commanding the frequency and then four more 
numbers that denote the duty cycle of the pulse width modulated signals used to command the speed of the wheels. The frequency and duty cycles are delimited by a comma. The receive buffer is parsed and each segment is converted into an integer to be passed into a function called writePWM function to send the pulse-width-modulated command to the robostix via the gumstix through i2c-io. The writePWM function takes in five integers: one frequency and four duty cycles, one for each wheel. This tells the robostix what frequency and duty cycle pulse-widthmodulated signal should be output via the output pins on the robostix. This writePWM function was written by three Electrical Engineering majors as a senior project.

The "mdir" string command communicates to the robostix which direction that the reaction wheels should spin. The "mdir" command consists of the "mdir" string and three integers: one motor number, and two bits that are either 0 or 1 . The bits determine the direction of the reaction wheel spin. A 0,0 commands the wheels to stop but continue to spin. A 0,1 commands the wheels to spin clockwise. A 1, 0 commands a counter-clockwise spin, and a 1,1 commands the wheels to brake. The receive buffer is parsed and each segment is converted from a string to an integer and then passed to the setMotorDirection function that sends the direction command to the reaction wheel motors from the robostix. The setMotorDirection function takes in eight integers: two integers for each of the four wheels, and this function was also created by the Electrical Engineers as their senior project with some modifications to allow for one command to command all four wheels instead of one wheel. The function uses system calls to the i2c-io program to set the appropriate bits for each motor direction command.

The "gyro" string command communicates to the robostix to read the analog to digital converters that are connected to the gyroscopes that read the $\mathrm{x}, \mathrm{y}$, and $\mathrm{z}$ angular velocities. The "gyro" command just consists of the string "gyro." For this command, five gyroscope readings 
are taken per call to this section of the gumstixServ function. The five readings are then averaged doing a simple mean. In order to get the readings from the gyroscopes, the desired analog to digital pin that the specific gyroscope in question is connected to is passed to the readADC function. So this function takes in an integer specifying the desired pin. The readADC function was also written by the Electrical Engineers for their senior project. To read the analog to digital pins, a system call is used in the function to read that pin. The readADC function returns an integer. The returned integer represents the digital representation of the input voltage to the pin, and these integers are then placed in a string output buffer. The buffer is finally written to the client socket for MATLAB to receive the gyroscope readings.

The "tach" string command communicates to the robostix to read the analog to digital pin that the tachometers are connected to. The command consists of just the string "tach." Reading the tachometers are different compared to the gyroscopes because the four tachometers are connected to only one analog to digital pin because the accelerometers that were originally going to be used on the PRWP used three analog to digital pins leaving only one pin left. So the "tach" command has to send a combination of three bits of high or low through three general input/output pins to get a certain tachometer reading. So for each tachometer, three bits are set to a certain output by system calls to i2c-io, depending on each wheel. Then the analog to digital converter pin for the tachometers is read giving the digital reading for how fast the reaction wheels are actually spinning. This process of setting the bits and reading the analog to digital pin is put in a for-loop that switches the bits to the appropriate output to get a different wheel tachometer each time. The readings are then placed in a string buffer and then written to the client socket so MATLAB can receive the wheel speed data. 
The "fbal" string command communicates to the gumstix, not the robostix because the serial port that connects to the CMFBS system microcontrollers is native to the gumstix. The "fbal" command instructs the fine balance motors on how much to spin to move the counterweights or what preprogrammed homing sequence to run. The "fbal" command consists of the word "fbal" and 3 double values that are the distances that each one of the fine balance motors need to move the counterweights. The receive buffer is parsed and each distance value is converted from a string to a float. Each value is the distance in meters that the counterweights are commanded to move. Each distance has to go through a number of conversions to translate the distance in meters to a motor value. The distance is divided by 0.0254 to convert them from meters to inches. Then that value is multiplied by 20 to convert from inches to revolutions (threads) because the lead screw has 20 threads per inch. Then this new value is the true revolutions of the lead screw which has to be converted to how much the motor has to spin to output one true revolution to the lead screw due to the 246:1 gearhead. The motor has to spin 246 times to output one revolution so the true revolutions are multiplied by a factor of 246 to get the amount of revolutions that the motor has to spin to get the desired distance in meters. According to the FAULHABER microcontrollers that control the CMFBS motors, a value of 1000 makes the motor spin one revolution so 1000 is multiplied to the converted value to get the value needed to be sent to the FAULHABER microcontroller to output the amount revolutions to spin the lead screw to move the counterweights the desired distance in meters. The converted command distances are then placed in one long connected string delimited by new line characters (In) because the FAULHABER microcontrollers have the transmission and receive (TX and RX) lines daisy chained together. Each command is preceded by a 0,1 , or 2 to indicate the node address of the FAULHABER microcontroller being communicated with. Also each command is 
preceded with an "hl3" command that tells the FAULHABER microcontroller to activate the limit switches to prevent the counterweights from running past the limit of the CMFBS tracks. This large string command is saved in a buffer and then sent to the serialCommConnect function. This function first configures the serial connection to the FAULHABER microcontrollers based on parameters needed by the FAULHABER microcontroller. The serialCommConnect function then opens the serial connection and then writes the command to the microcontroller. Once the command is written, the connection is closed.

The last case of the if-statement is the "q" command string, which just consists of the letter "q." This command closes both the client and the listening socket. Once this command is executed, the server is put back into listening mode to wait for another command from the ground computer.

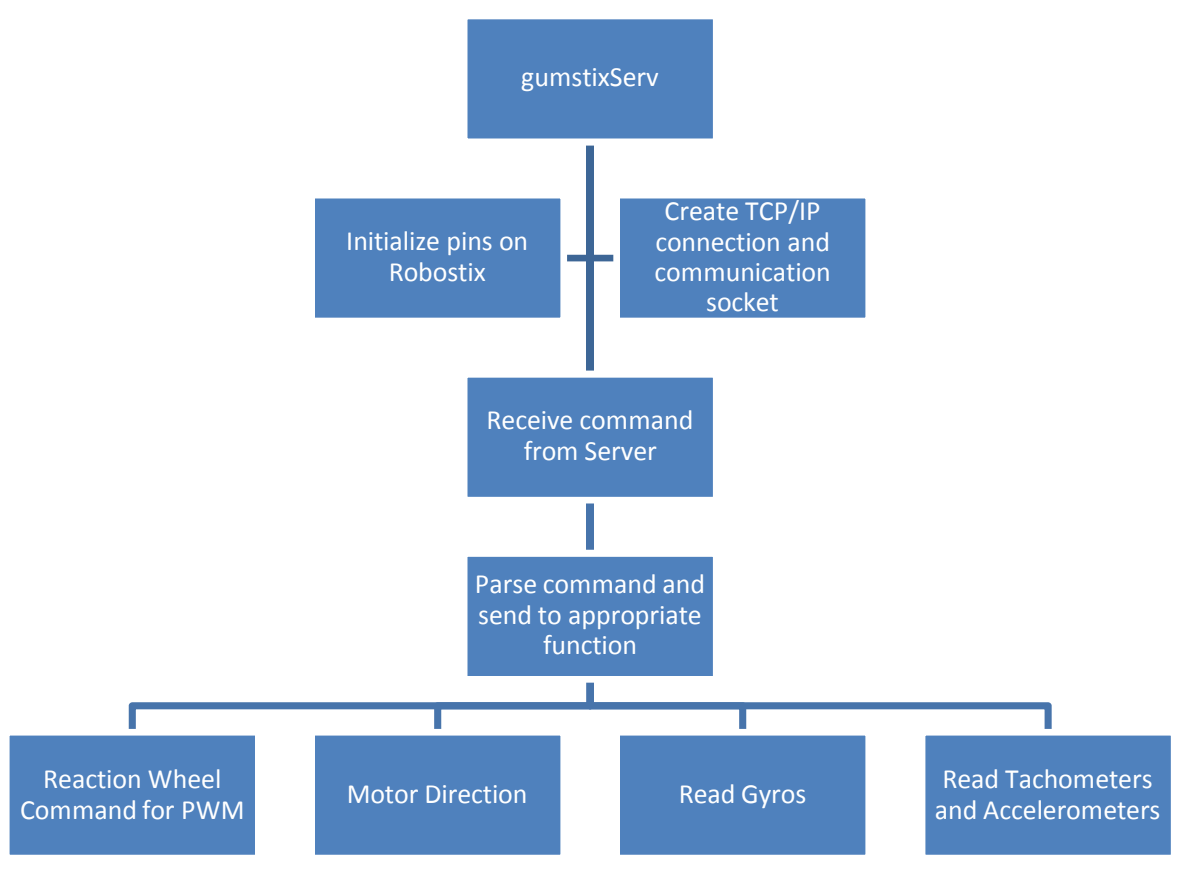

Figure 19: gumstixServ server software flow diagram 


\section{3 i2c-io}

The program, i2c-io serves as an interface program between the robostix and the gumstix. This is an open source program that is used for communication to and between microcontrollers. It is a multi-master serial computer bus, and it allows low-speed devices to connect to an embedded system. ${ }^{11}$

\subsection{MATLAB/SIMULINK}

The programs, MATLAB and SIMULINK, are utilized to do the analysis necessary for the system identification method. MATLAB is used to compute and analyze the data received and to output a system identified matrix. SIMULINK is used to command the reaction wheels, and read the sensor data of the PRWP. The sensor data is then used to create a system identified matrix containing the inertias and $\mathrm{CM}$ locations. The $\mathrm{CM}$ locations are then used to move the CMFBS counterweights to try to bring the CM to the pivot point of the platform.

\subsection{1 gumstixSend.m}

In order to send commands to the gumstix from SIMULINK, a send block created by an S-function block was created to send commands via the TCP/IP Bluetooth link between the ground computer and the gumstix. So the function, gumstixSend. $m$ is created to act as a client block for SIMULINK. The function first initializes the receive block and determines port width and number of ports the block will have. This function then creates a TCP/IP connection through MATLAB, and then opens and connects to the server socket. In the "Source Block Parameter" box, the parameter, "rwcomm" or "fbal" is typed in to tell what function the block will perform; whether it will be a reaction wheel command or a fine balancing command.

\footnotetext{
11 "I2C-Bus: What's That?" I2C-Bus: What's That? Web. <http://www.i2c-bus.org/>.
} 
If the "rwcomm" parameter is present, the input port of the block is parsed. The first four digits of input data determine whether the wheels have to brake before initiating a new reaction wheel command. This is necessary to prevent too much stress on the motors. An integer of 0 brakes the wheels before the new command, and the digit 1 allows the wheels to keep spinning even when the new command is sent. If the digit is 0 , then the gumstixSend.m function will send a motor direction command to brake the wheels.

The next four inputs are angular velocities for each wheel. The angular velocities are converted to frequency and duty cycles for each wheel through the freqDC function. The frequency of the desired pulse-width-modulated signal that the reaction wheel command is sending is set to $1000 \mathrm{~Hz}$. Once, the angular velocities are converted, they are placed in a string buffer. The buffer is then written to the gumstix via the Bluetooth TCP/IP communication socket.

The "fbal" parameter informs the gumstixSend.m function to send a fine balancing command to move the counterweights the distance that is desired. The desired distance is input through the block's input port. Three inputs are parsed from the port, which are the three distances that the fine balancing counterweights must move. The three distances are then placed into a string buffer, and this buffer is then written to the gumstix via the Bluetooth TCP/IP communication socket.

\subsection{2 gumstixRec1.m}

Data is received by MATLAB and SIMULINK through a receive block created by an Sfunction block similar to the send block called gumstixRec1.m. The function first initializes the receive block and determines port width and number of ports the block will have. The gumstixRec1.m function then creates a TCP/IP connection through MATLAB. A socket is 
opened and the socket is connected to the gumstix server socket. The function then sends a string with the word "gyro" to the server to initiate a gyroscope read. The data sent back by the server is then saved. Once the data is received the receive block send a string command to the server, with the word "tach" to initiate the reading of the tachometers. The server then will send the data back and the data is stored. The gyroscope and tachometer data is then placed in an array and the output through the receive block output. The connection is then closed with the server.

\subsubsection{System ID SIMULINK Simulation}

The System ID SIMULINK simulation (systemIDReal.mdl) program sends commands to the reaction wheels to disturb the system and then receives data from the sensors on the PRWP to compute a system identified matrix. The System ID simulation has three distinct parts, a SIMULINK execution control timer and clock, a send portion that outputs reaction wheel commands, and then a receive portion that reads in data from the PRWP sensors.

The SIMULINK execution control timer forces SIMULINK to run in real time. If this control timer was not in the simulation, SIMULINK will try to run as fast as possible causing errors in the discrete derivatives being taken. Forcing SIMULINK to run at real time allows the ground computer to get real time data acquisition and send real time commands. This program was made by a user in the MATLAB online community and was available on the internet through MATLAB's Central File Exchange. A clock is used to keep track of the time in the simulation so when the simulation gets to a certain time, the signal being routed to the reaction wheels can be changed to a different signal.

The send portion of the System ID simulation contains 8 discrete sine wave generators that are out of phase of each other by 90 degrees of each other. This causes the platform to spin 
around the z-axis. The discrete sine wave generators are in two groups of four, one sine wave generator for each reaction wheel. The first group of sine wave generators has a positive bias while the second group has a negative bias. After a number of seconds, the input for the reaction wheel command is switched from the positive bias group to the negative bias group causing the wheels to spin in the opposite direction. The 4 sine waves per group are muxed together into one signal and then go into a gain to amplify the signals. The signal is then input into the send block to be sent to command the reaction wheels.

The receive portion uses the receive block to get data from the gumstix/robostix combination. This block outputs the gyroscope readings, tachometer readings, and the accelerometer data (whenever an accelerometer is integrated into the PRWP). Each reading is sent through a calibration block to calibrate the gyroscopes and the tachometer readings. The acceleration data is derived from the gyroscope data sending the gyroscope readings through a discrete time difference block. The Euler angles are derived by sending the gyroscope readings through a discrete time integrator. Each calibrated or derived value is then placed into an A matrix which is used in the system identification process to obtain the identified properties of the system. The A matrix is then output to the workspace. The calibrated tachometer data is also used to compute the body torque matrix necessary for the system id process. The torque data is then computed to convert the wheel speeds to body torque. The torque matrix for the PRWP is then outputted to the workspace.

\subsection{4 systemIDStart.m}

The systemIDStart $\mathrm{m}$-file is an automated program that runs the system ID process. The first thing that the $\mathrm{m}$-file does is that it defines the parameters of the wheel inertia and other constants. Also the m-file runs the systemIDReal simulation multiple times to get a better 
average for the A matrix, which provide a better result. The user can define the amount of seconds that each run of the systemIDReal will take and also the amount of iterations that the SystemIDReal will be run. The system identification process is an iterative process, so running the simulation multiple times is necessary to achieve balance of the CM.

So, the systemIDReal simulation is initiated and the A matrix and the torque matrix is output to the workspace. Then systemIDStart takes the A matrix and the torque matrix and then computes the identified system matrix. This matrix will be a 9x1 with the last 3 values in the matrix being the torque induced by the CM being offset from the pivot point of the PRWP. The systemIDStart function then computes how far the $\mathrm{CM}$ is from the pivot point by dividing the mass of the PRWP and the gravity constant from the induced torque. The resulting values will are then divided by two and the negative of these values are sent to the $F B a l$ simulation to command the distances for the counterweights to move to try to balance out the CM. The reason why the negative of half of the distance that the $\mathrm{CM}$ is off by is given as the distance the counterweights have to move is because moving the counterweights in the opposite direction will allow the weights to counteract that excess mass in that one direction. 


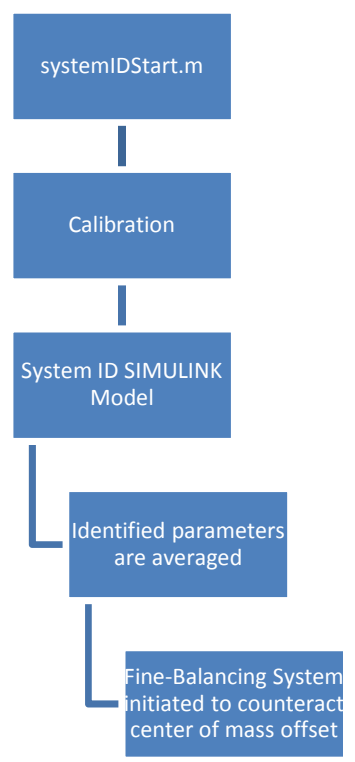

Figure 20: System ID Simulation Runtime Process 


\section{Chapter 4: Theoretical and Experimental Analysis}

In this chapter, the theoretical analysis is explained in more depth. Furthermore, experimental analysis is described in more detail to show how the theoretical analysis is tested experimentally.

\subsection{Theoretical System Identification Analysis}

In order to verify the feasibility of system identification for the PRWP, the theoretical analysis would have to be compared to the experimental analysis produced in this thesis. The theoretical model and analysis is from Patrick Healy's thesis referred to before in this paper. ${ }^{12}$ The PRWP system of equations is shown in the equation below:

$$
\vec{r} \times \underline{R}_{E} \cdot m \vec{g}=\underline{I} \cdot \dot{\vec{\omega}}+\vec{\omega} \times \underline{I} \cdot \vec{\omega}+\sum_{\ell=1}^{4}\left(R_{W \ell} \cdot\left[\begin{array}{c}
I_{11}^{W \ell} \cdot \dot{\omega}_{W \ell} \\
0 \\
0
\end{array}\right]+\vec{\omega} \times R_{W \ell} \cdot\left[\begin{array}{c}
I_{11}^{W \ell} \cdot \omega_{W \ell} \\
0 \\
0
\end{array}\right]\right)
$$

His thesis goes on to explain how these equations are manipulated to form an identified state matrix, which is shown below:

$$
x=\left[\begin{array}{lllllllll}
I_{x x} & I_{x y} & I_{x z} & I_{y y} & I_{y z} & I_{z z} & m g r_{x} & m g r_{y} & m g r_{z}
\end{array}\right]^{T}
$$

The system identification process uses the regression model shown below:

$$
0=A x-T
$$

\footnotetext{
${ }^{12}$ Healy, Patrick B.
} 
Using this regression model, you can solve for the state matrix taking the inverse of the A matrix and adding the torque matrix. The A matrix after manipulating the platforms system of dynamic equations is shown below:

$$
A=\left[\begin{array}{ccccccccc}
\dot{\omega}_{x} & \left(\dot{\omega}_{y}-\omega_{x} \omega_{z}\right) & \left(\dot{\omega}_{z}+\omega_{x} \omega_{y}\right) & -\omega_{y} \omega_{z} & \left(\omega_{y}^{2}-\omega_{z}^{2}\right) & \omega_{y} \omega_{z} & 0 & -C \theta_{1} C \theta_{2} & S \theta_{1} C \theta_{2} \\
\omega_{x} \omega_{z} & \left(\dot{\omega}_{x}+\omega_{y} \omega_{z}\right) & \left(\omega_{z}^{2}-\omega_{x}^{2}\right) & \dot{\omega}_{y} & \left(\dot{\omega}_{z}-\omega_{x} \omega_{y}\right) & -\omega_{x} \omega_{z} & C \theta_{1} C \theta_{2} & 0 & S \theta_{2} \\
-\omega_{x} \omega_{y} & \left(\omega_{x}^{2}-\omega_{y}^{2}\right) & \left(\dot{\omega}_{x}-\omega_{y} \omega_{z}\right) & \omega_{x} \omega_{y} & \left(\dot{\omega}_{y}+\omega_{x} \omega_{z}\right) & \dot{\omega}_{z} & -S \theta_{1} C \theta_{2} & -S \theta_{2} & 0
\end{array}\right]
$$

The external and body torque matrix is shown below as well:

$$
\begin{aligned}
T & =-d=-\left[\begin{array}{cccc}
C \beta & 0 & -C \beta & 0 \\
0 & C \beta & 0 & -C \beta \\
S \beta & S \beta & S \beta & S \beta
\end{array}\right]\left[\begin{array}{c}
I_{11}^{W 1} \dot{\omega}_{\pi 1} \\
I_{11}^{W 2} \dot{\omega}_{W 2} \\
I_{11}^{W 3} \dot{\omega}_{W 3} \\
I_{11}^{W 4} \dot{\omega}_{W 4}
\end{array}\right] \\
& +\left[\begin{array}{cccc}
\omega_{y} S \beta & \left(\omega_{y} S \beta-\omega_{z} C \beta\right) & \omega_{y} S \beta & \left(\omega_{y} S \beta+\omega_{z} C \beta\right) \\
\left(\omega_{z} C \beta-\omega_{x} S \beta\right) & -\omega_{x} S \beta & -\left(\omega_{x} S S \beta+\omega_{z} C \beta\right) & -\omega_{x} S \beta \\
-\omega_{y} C \beta & \omega_{x} C \beta & \omega_{y} C \beta & -\omega_{x} C \beta
\end{array}\right]\left[\begin{array}{c}
I_{11}^{W 1} \omega_{W 1} \\
I_{11}^{W 2} \omega_{W 2} \\
I_{11}^{W 3} \omega_{W 3} \\
I_{11}^{W 4} \omega_{W 4}
\end{array}\right]
\end{aligned}
$$

The first term contains the torque due to the spinning of the reaction wheels, and the second term is the torque due to the dynamics of the platform. A least squares estimation is used to estimate the state. For every time step the A and T matrices will be stored in a large A and T matrix that consists of each A and T matrix concatenated vertically per time step. This is shown below: 


$$
\begin{gathered}
\bar{A}=\left[\begin{array}{c}
A_{1} \\
A_{2} \\
\vdots \\
A_{k}
\end{array}\right] \\
\bar{T}=\left[\begin{array}{c}
T_{1} \\
T_{2} \\
\vdots \\
T_{k}
\end{array}\right]
\end{gathered}
$$

The $\mathrm{k}^{\text {th }}$ time step refers to the last time step that is run for the system identification simulation. Since the A matrix is not a square matrix and the inverse needs to be taken, a pseudo-inverse is used to solve for the state in the regression model.

The physical system, for the example of a spacecraft that is identified consists of the inertia terms in an inertia matrix and the system's torque induced by misalignment of the center of mass to the center of rotation of the craft. Through that induced torque, the location of the center of gravity can be easily determined because this torque consists of the product of the local gravity, mass, and the moment arm between the center of mass (CM) and the center of rotation (CR). Through dividing the induced torque by the mass and the moment arm, the location of the center of mass is determined. Most of the analysis performed is detailed in Patrick Healy's Thesis paper. ${ }^{13}$ He goes into detail on the mathematics of the system identification.

For this thesis, the identified distance between the $\mathrm{CM}$ and the $\mathrm{CR}$ is used to balance the PRWP. Half this distance is commanded to move counterweights in the opposite direction of the imbalance to attempt to center the PRWP. Once the PRWP is balanced, the inertia matrix

\footnotetext{
${ }^{13}$ Healy, Patrick B. "Mass Property System Identification of a Spacecraft Simulator." Thesis.
} 
identified by the system identification process is used for the PRWP to fully optimize the main control system of the simulator.

Healy ran a control simulation based on the dynamic equations of motion of the PRWP. This simulation is run for 150 seconds in simulation time and simulated state data is recorded. Also the body torques for the PRWP are recorded as well in order to compute the theoretical system identified matrix. The center of mass for this simulation is offset by $[-0.0165,0.04,-$ $0.5656 \mathrm{~cm}$ from the center of rotation (the pivot point at the air-bearing). With this offset, the state data (angular velocity, angular acceleration, and Euler angles) are recorded as well as the body torques on the platform necessary to command the PRWP from the origin at the pivot point to a quaternion orientation of $[0.01,0.01,0.7,0.714]$. Healy's simulation also included simulated white noise that a real system would encounter due to electrical interference and wiring.

The recorded state and torque data is then used to find the identified state matrix by performing a pseudo-inverse on the A matrix (composed of the PRWP's state data) and multiplying it by the T matrix, which consist of the torques applied to the system by the reaction wheels and the body torques experienced by the PRWP. The identified state matrix consists of a new identified Inertia matrix as well as the induced torque due the misalignment of the center of mass with the center of rotation. The induced torque terms are then divided by the mass of the PRWP and the gravity term to obtain the center of mass offset.

\subsection{Experimental Analysis}

The experimental application of system identification is performed on the PRWP. This analysis did not use the same torque profile as the theoretical analysis performed by Healy. The torque profile is generated by commanding the speeds of each wheel, the wheel rates, with 
values from discrete sine wave generators that were out of phase with each other. Each wheel has a corresponding sine wave generator. The out of phase sine waves allows the PRWP to be perturbed allowing the platform to move and nutate due to the misalignment of the wheel speed command sine waves. The TACs are used to get true wheel rates at the time the state data (angular velocity) is recorded. The TAC data is needed to more accurately perform system identification because the wheel speeds that are commanded to the reaction wheels are not the actual speeds of the reaction wheels at the time of data measurement because there is a lag time between when the wheels receive a speed command and when they actually reach the commanded speed. This lag is due to the reaction wheels motor response time and practical motor acceleration.

The wheel rates that are recorded from the TACs have a discrete derivative taken from it to calculate the wheel accelerations for each reaction wheel. These wheel accelerations are used to calculate the total torque experienced by the PRWP. Due to the noisiness of the data from the TACs, the derived wheel accelerations are averaged using a weighted averaging scheme to attempt to eliminate the noise. The wheel rate data requires no such averaging scheme because the noise is relatively low, but due to the discrete derivative that low noise is amplified thus requiring an average to be taken for the wheel accelerations.

The sensors are read to compile the A matrix that will, with the T matrix, provide a solution for the system identification process. The sensors read for the PRWP are the gyroscopes that indicate the angular velocities experienced by the platform. Since the gyroscopes are the only sensors presently on the PRWP to indicate body movement, their data is used to derive the acceleration and position data for the platform. The gyroscope data is averaged using a similar weighted average scheme that is used for the wheel accelerations due to the noise level in their 
readings. The gyroscope readings have discrete derivatives taken to produce angular acceleration readings and due to the noise experienced by the gyroscope readings, the derived angular acceleration data contain a weighted average scheme applied to it as well.

Once the A matrices and T matrices are composed for each time step, the system identification process described before is applied using these matrices. The A matrices are concatenated vertically and so is the T matrices as well. The T matrix is then multiplied by the pseudo-inverse of the concatenated A matrices to yield the identified state matrix. The new inertias are the first six terms in the state vector and the Center of Mass offset can be extrapolated from the last three terms in the identified state vector.

Once the new CM locations are found, the CMFBS is commanded to fine balance the platform. The command sent commands each axis to move the counterweights one-fourth of the $\mathrm{CM}$ offset found by system identification. 


\section{Chapter 5: Results}

The following chapter describes the performance of the handling algorithm results as well as the experimental analysis data obtained from running the System Identification process on the PRWP. Also the Theoretical Analysis results are compared to the Experimental Analysis results to verfify the validity of the System Identification process for a 4-wheel reaction wheel platform. Finally the errors associated with the experimental results are discussed to explain for an error associated with the final results of the experimental analysis.

\subsection{Handling Algorithm System}

For system identification to be feasible on the PRWP, a communication handling algorithm is needed for the system to be functional. Without an input/output system active on the control platform, commands cannot be sent to it and data cannot be sent from it from the ground computer. The gumstixServ algorithm serves as the handling algorithm for commands to be sent and data to be transmitted from the PRWP. As described before in the previous chapter, this server allows for a TCP/IP connection to be utilized via the Bluetooth connection so MATLAB on the ground computer can send reaction wheel commands and get gyroscope and tachometer data from the PRWP.

The gumstixServ server on the gumstix has been able to send and receive data at a $1 \mathrm{~Hz}$ rate. This translates to the ability, for a one second interval, to send one set of commands to each of the four reaction wheels and read in one set of sensor data, which include gyroscope rates, tachometer readings, and eventually accelerometer data. The server tends to crash if it is queried at any higher frequency. It starts to have an input error receiving commands from the ground computer. If the commands are sent too fast from the ground computer to the gumstix, the 
server's buffer gets saturated and then does not have enough time to parse the data before new commands are received. Once the buffer gets saturated, the received commands continue to get concatenated on top of each other and then the server crashes. After this, the server needs to be terminated, and then gumstix will need to be rebooted.

One of the possible reasons for the server to crash is the extensive use of the system() command to communicate with i2c-io. This command is very time intensive as well as processor intensive. It seems to slow the server down greatly. Due to this limitation, the readADC function in the server that uses the system() command has to be limited in its use, thus preventing tachometer readings from further being averaged. When the tachometer readings were averaged over ten times per call to the "tach" portion of the server, the server would crash a few seconds into the system identification simulation, but when they were decreased to around six times per call, the server would hardly crash. This limitation also prevents simulations to run at an exceedingly long time.

\subsection{Verification of Experimental Simulation Process}

In order to verify the experimental simulation process described in the Experimental Analysis section, the torque, angular velocity, angular acceleration, and Euler angle data is taken from Healy's simulation and used as the input to the experimental simulation. By using the theoretical data in the experimental simulation algorithm, the output from the experimental algorithm can be used to compare to the output from the theoretical analysis to determine whether the experimental algorithm performs within an acceptable range of error to the theoretical ideal result. The wheel speeds, in radians/sec, for each wheel were extrapolated from the torque data from Healy's theoretical simulation. The extrapolated wheel speeds were then 
used to calculate the wheel accelerations for each wheel via the same discrete derivative used in the experimental simulation.

Ultimately, Healy's data from the theoretical system identification analysis was inputted into the experimental simulation algorithm to create the A and T matrices for each time step. The system identification process was applied to these matrices to calculate the identified sate matrix. The identified state matrix from the experimental simulation using the theoretical simulation data is then compared to the fully theoretical simulated identified state matrix found in Healy's simulation. In Figure 21, the theoretical identified state matrix is compared to the identified state matrix computed using the experimental simulation algorithm using the theoretical state and torque data. The percent differences are relatively low, from $0.028 \%$ to $0.75 \%$, for the principle inertias, but the error is quite significant for the non-principle inertias, but the magnitude of their values are small so a small change in value would result in a greater percentage difference. The last three terms of the experimental identified state matrix, the induced torque due to the misalignment of the center of mass with the pivot point, have a less than a tenth of a percent difference compared to the theoretical identified state matrix terms. This very beneficial to this thesis because those three terms will be used to command the CMFBS to balance out the CM offset.

\begin{tabular}{|c|c|c|c|}
\hline Identified State & $\mathrm{x}$ theoretical & $\mathrm{x}$ experimental & \% difference \\
\hline $\mathrm{I}_{\mathrm{xx}}$ & 0.6499 & 0.6548 & 0.75 \\
\hline $\mathrm{I}_{\mathrm{xy}}$ & 0.000458 & -0.0051 & 108.9 \\
\hline $\mathrm{I}_{\mathrm{xz}}$ & -0.000540 & -0.000498 & -8.39 \\
\hline $\mathrm{I}_{\mathrm{yy}}$ & 0.6001 & 0.5980 & -0.35 \\
\hline $\mathrm{I}_{\mathrm{yz}}$ & 0.0015 & 0.000524 & -187.8 \\
\hline $\mathrm{I}_{\mathrm{zz}}$ & 0.5598 & 0.5597 & -0.028 \\
\hline $\mathrm{mgr}_{\mathrm{x}}$ & 0.0405 & 0.0405 & 0.043 \\
\hline $\mathrm{mgr}_{\mathrm{y}}$ & -0.0981 & -0.0982 & 0.058 \\
\hline $\mathrm{mgr}_{\mathrm{z}}$ & 1.3872 & 1.3878 & 0.047 \\
\hline
\end{tabular}




\section{Figure 21: Theoretical identified state matrix compared to experimental identified state matrix using theoretical rate data}

The principle inertias and the induced torque terms are less than one percent in difference from the theoretical analysis, and the non-principle inertias are so low in magnitude, they are almost negligible in change. The error is attributed to integration and derivative error because the theoretical data has 7 time steps for the first 0.1 seconds, and then shifts to one time step per 0.1 seconds. Therefore, through this analysis of using the theoretical state data as the input to the experimental simulation algorithm, the experimental algorithm is verified.

\subsection{Theoretical and Experimental Analysis Comparison}

The principle inertias were calculated using the experimental setup of perturbing the PRWP with phased offset sine waves commanded wheel speeds. These experimental results are shown in Figure 22 in the third column of the table. The true experimental identified state matrix differs greatly from the identified state matrix from the theoretical analysis shown in the second column of the figure. The difference can be explained due to the great noise that is seen in the angular velocity, angular acceleration, and wheel acceleration data. As stated before, those data sets had to be averaged to attempt to eliminate the noise, and due to this averaging, there is great error introduced. Also, with the angular accelerations being derived from the angular velocities, when taking the discrete derivative introduces more error, which is coupled with the inherent noise experienced from the angular velocity data. One other explanation for this error is the low frequency of sensor data acquisition from the PRWP. The $1 \mathrm{~Hz}$ frequency (one set of data per second) allows more error to be added into the calculations. The low frequency is not able to pick up the smaller modes of oscillation of the PRWP during the perturbations, and when this data has an integral or derivative taken on it, the error is magnified into the derived values. The $1 \mathrm{~Hz}$ limitation is a system limitation that could not be overcome with the present state of the 
onboard computer and interface between the data acquisition chip (robostix) and the onboard computer (gumstix).

\begin{tabular}{|c|c|c|c|}
\hline Identified State & $\mathrm{x}$ theoretical & $\mathrm{x}$ true exp & \% difference \\
\hline $\mathrm{I}_{\mathrm{xx}}$ & 0.6499 & -0.2094 & 410.4 \\
\hline $\mathrm{I}_{\mathrm{xy}}$ & 0.000458 & -0.1351 & 100.3 \\
\hline $\mathrm{I}_{\mathrm{xz}}$ & -0.000540 & -0.0750 & 99.3 \\
\hline $\mathrm{I}_{\mathrm{yy}}$ & 0.6001 & 0.1940 & -209.3 \\
\hline $\mathrm{I}_{\mathrm{yz}}$ & 0.0015 & -0.1434 & 101.1 \\
\hline $\mathrm{I}_{\mathrm{zz}}$ & 0.5598 & 0.5802 & 3.5 \\
\hline
\end{tabular}

Figure 22: Comparison of theoretical and experimental identified state matrices

The experimental analysis shows converged principle inertias. In Figure 23, the principle inertia around the $\mathrm{x}$-axis theoretical convergence history is shown. The settling time for Ixx is a little over 1 second with an average value of $0.6499 \mathrm{~kg} / \mathrm{m}^{2}$. In Figure 24, the experimental convergence history is shown on top with a magnified convergence history plot below. The Inertia is identified after about 10 seconds with a value of $-0.2094 \mathrm{~kg} / \mathrm{m}^{2}$, which is virtually impossible to achieve with our knowledge of physics because a negative inertia equates to negative mass. There are probably causes for this discrepancy that will be discussed later in this thesis. 


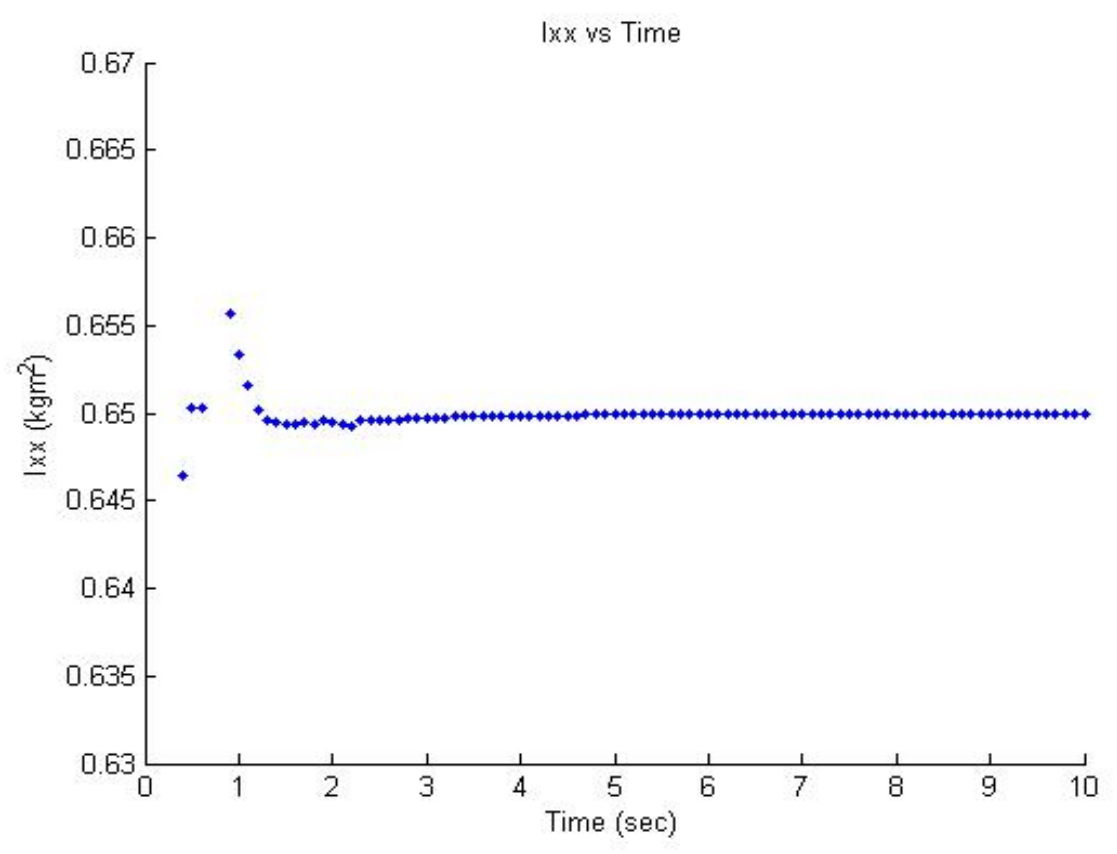

Figure 23: Theoretical convergence of Ixx
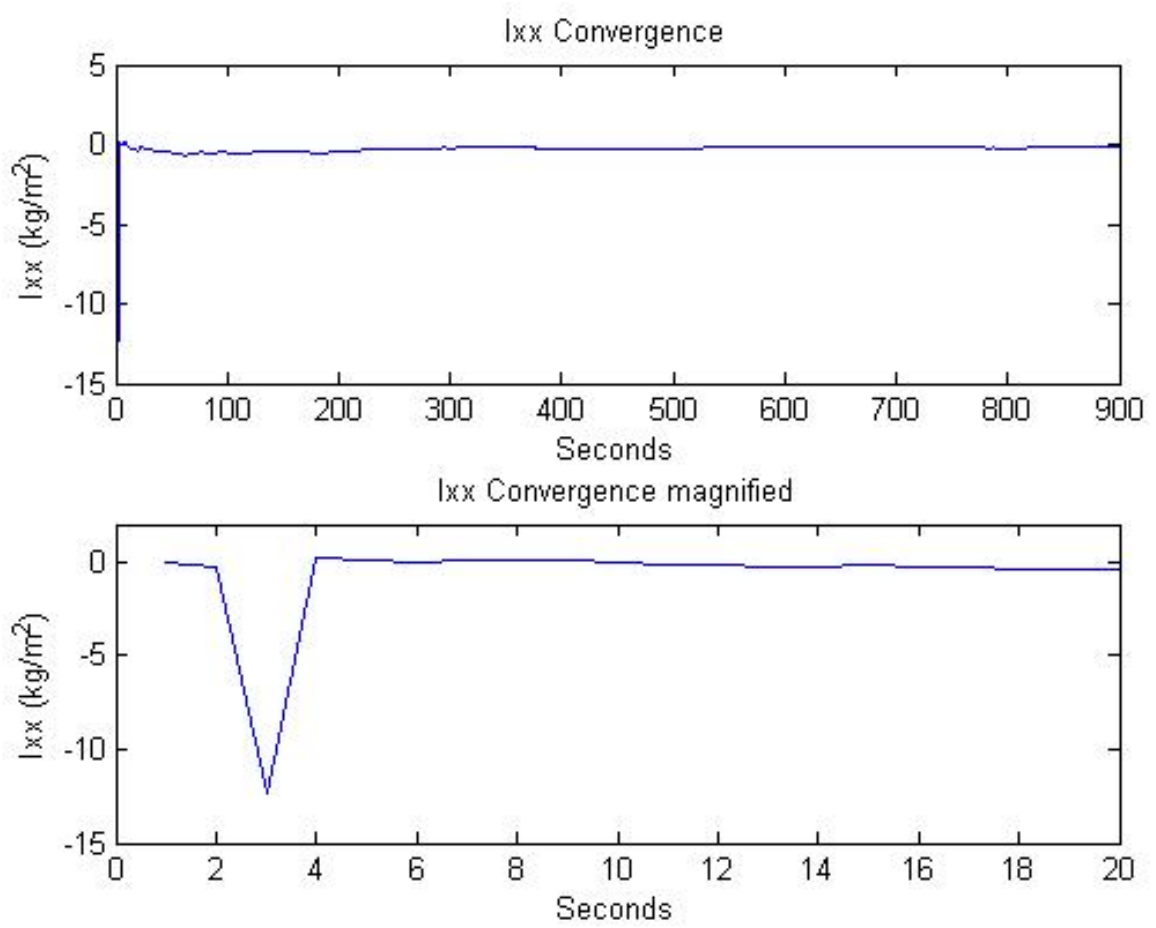

Figure 24: Experimental convergence of Ixx (top - full run; bottom - magnified) Figure 25 shows the convergence history of Iyy through the theoretical system identification analysis. The value of Iyy settles around 3 seconds or so at a value of 0.6001 
$\mathrm{kg} / \mathrm{m}^{2}$. In Figure 26, the convergence history for Iyy obtained from the PRWP via the experimental system identification simulation shows a rough settling time of about 200 seconds with a value of $0.1940 \mathrm{~kg} / \mathrm{m}^{2}$.

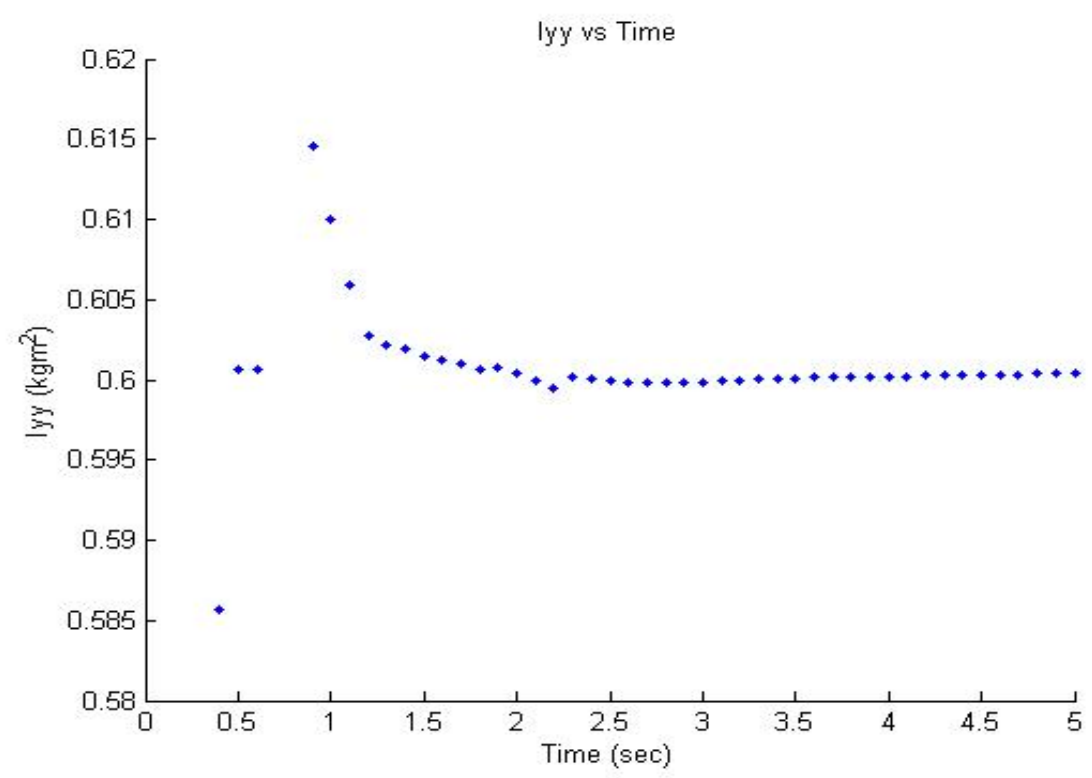

Figure 25: Theoretical convergence of Iyy

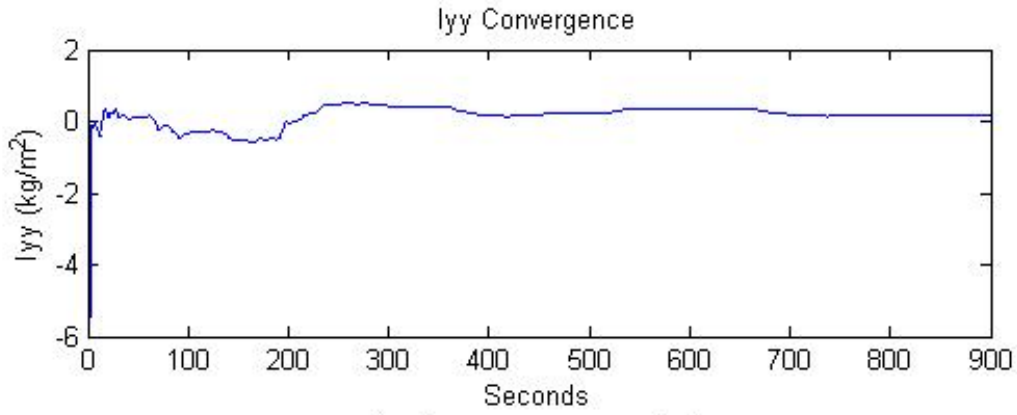

lyy Convergence magnified

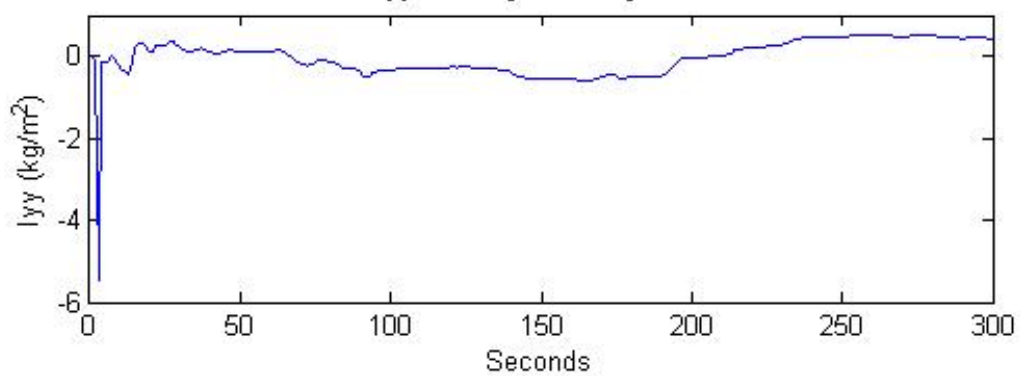

Figure 26: Experimental convergence of Iyy (top - full run; bottom - magnified)

Figure 27 shows the convergence history of Izz through the theoretical system

identification analysis. The value of Izz settles after about 1 second at a value of $0.5598 \mathrm{~kg} / \mathrm{m}^{2}$. 
In Figure 28, the convergence history for Izz obtained from the PRWP via the experimental system identification simulation shows a rough settling time a little over 200 seconds, but with a value of $0.5802 \mathrm{~kg} / \mathrm{m}^{2}$. The Izz value is closer to the actual value realized by the theoretical analysis because there is more movement about the $\mathrm{z}$-axis because there are no boundaries for that axis on the PRWP. The more movement allows the data to be richer in the $\mathrm{z}$-axis therefore better dampening out the noise experienced by the gyroscopes readings.

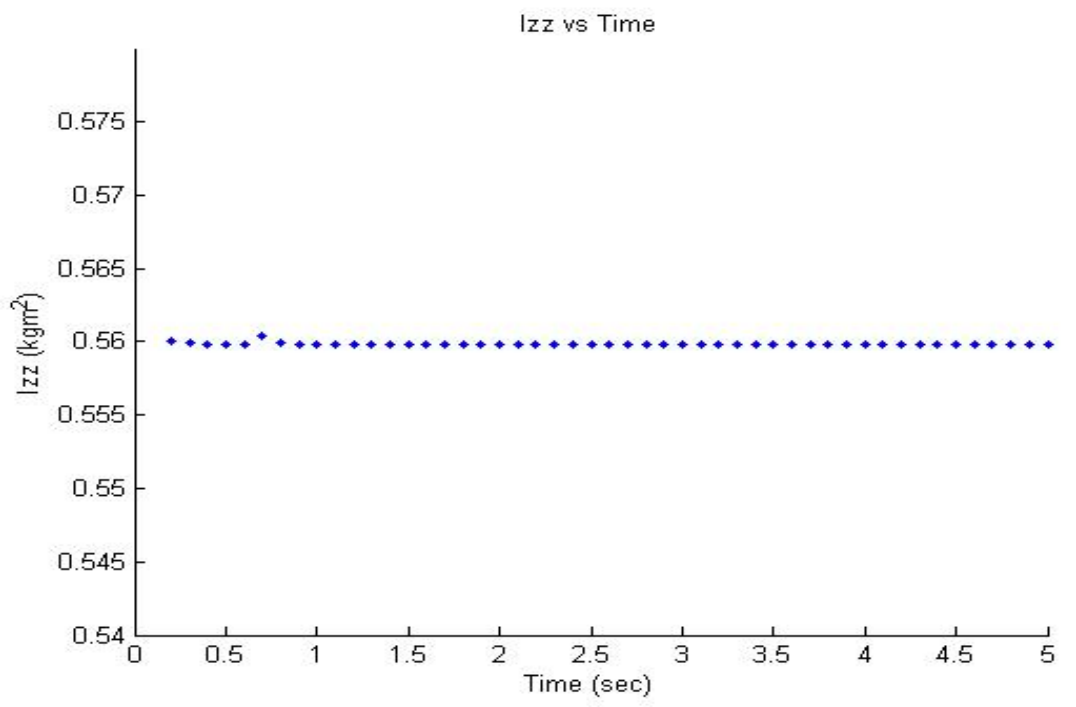

Figure 27: Theoretical convergence of Izz 

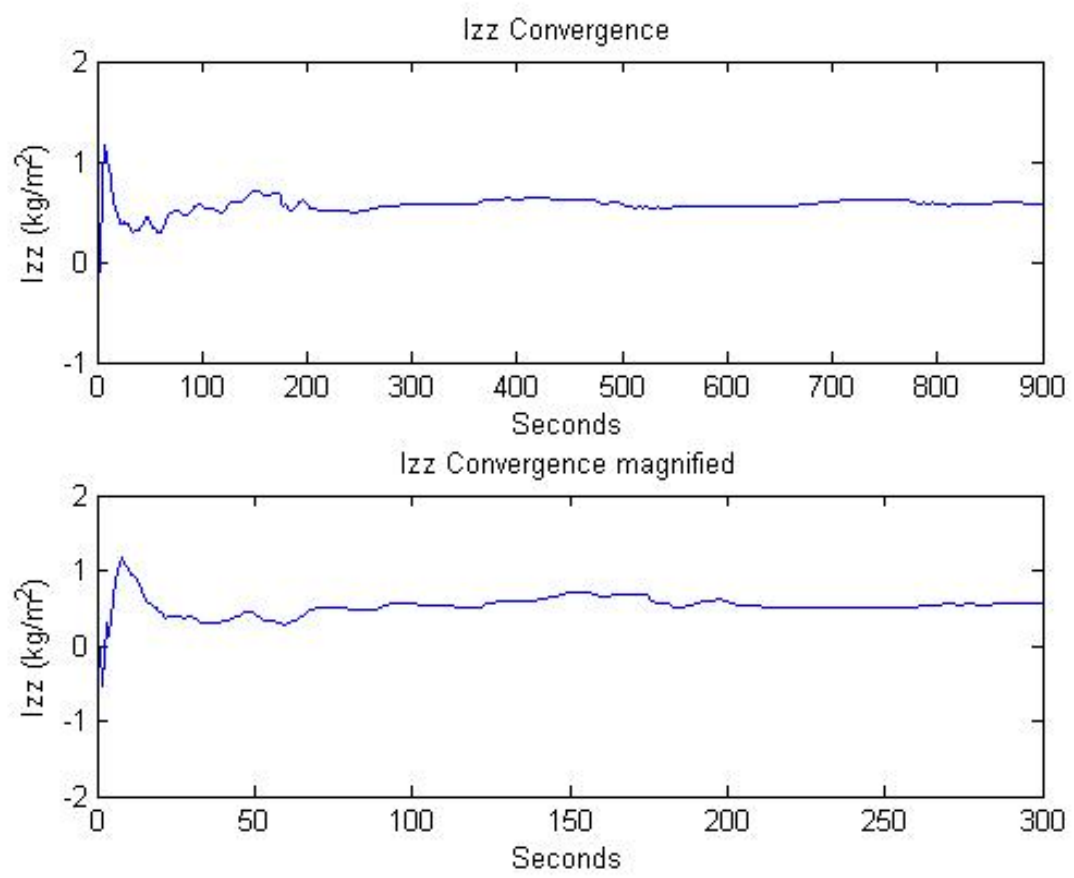

Figure 28: Experimental convergence of Izz (top - full run; bottom - magnified)

The induced torque solution from the system identification process (the last three terms in the system identified matrix) will be used to calculate the center of mass offset needed by the CMFBS to balance the PRWP. The system identification process is run yielding the system identified matrix. Once the derived CM offset calculation is used to counterweigh the PRWP, the system identification process will be run again to yield a new identified matrix that will be used to run the CMFBS again to counterweigh the PRWP. Running the system identification process over and over again while using each new $\mathrm{CM}$ offset calculation to counterweigh the offset using the CMFBS is an iterative process allowing the PRWP to be finely balanced.

The experimental system identification CM offset calculations that are used to command the CMFBS seem to balance the PRWP. After running several runs of the system identification process and running the CMFBS, the PRWP is able to be put into an orientation and it stays in place for about one second before swinging back with the motion of a pendulum. The more balanced the PRWP becomes the slower the pendulum motion becomes. So each time the 
system identification process is run, the PRWP's pendulum motion becomes slower and slower indicating that the PRWP is actually being balanced.

Even though the experimental identified inertias have great error, the induced torque terms in the system identified matrix has less relative error compared to the experimental identified inertias because the Euler angles will be used to compute the induced torque values in the system identified matrix. The last three columns in the A matrix, which will be used to compute the induced torque terms in the system identified matrix, are comprised of the Euler angles. The angular velocity data of the gyroscopes are integrated to produce the Euler angles that will be needed and because an integral is being taken place, there is less error associated to the Euler angles as opposed to the angular accelerations that will be derived from taking a derivative of the experimental angular velocity data from the gyroscopes.

\subsection{Errors}

Noise in the measurements contributed to the errors in the data, and they could be the reason why the principle inertia about the $\mathrm{x}$-axis is found to be negative through the experimental process. In Figure 29, the raw angular velocity is displayed, and it shows how noisy the data is. Instead of smooth curves for each axis' angular velocity data curve, the curve oscillates erratically due to the noise experienced by the PRWP gyroscope measurements. The noise is attributed to the inaccuracy that is caused by voltage irregularities when the analog to digital converters are read on the robostix by the server program on the PRWP. A five millivolt discrepancy can equal one ADC reading (the numerical reading that interprets the voltage into a numerically reading). Usually there is about 10 to 15 millivolt oscillation and sometimes more depending on the rate of change of the PRWP at the time. This oscillation creates those spikes in the data seen in Figure 29. 


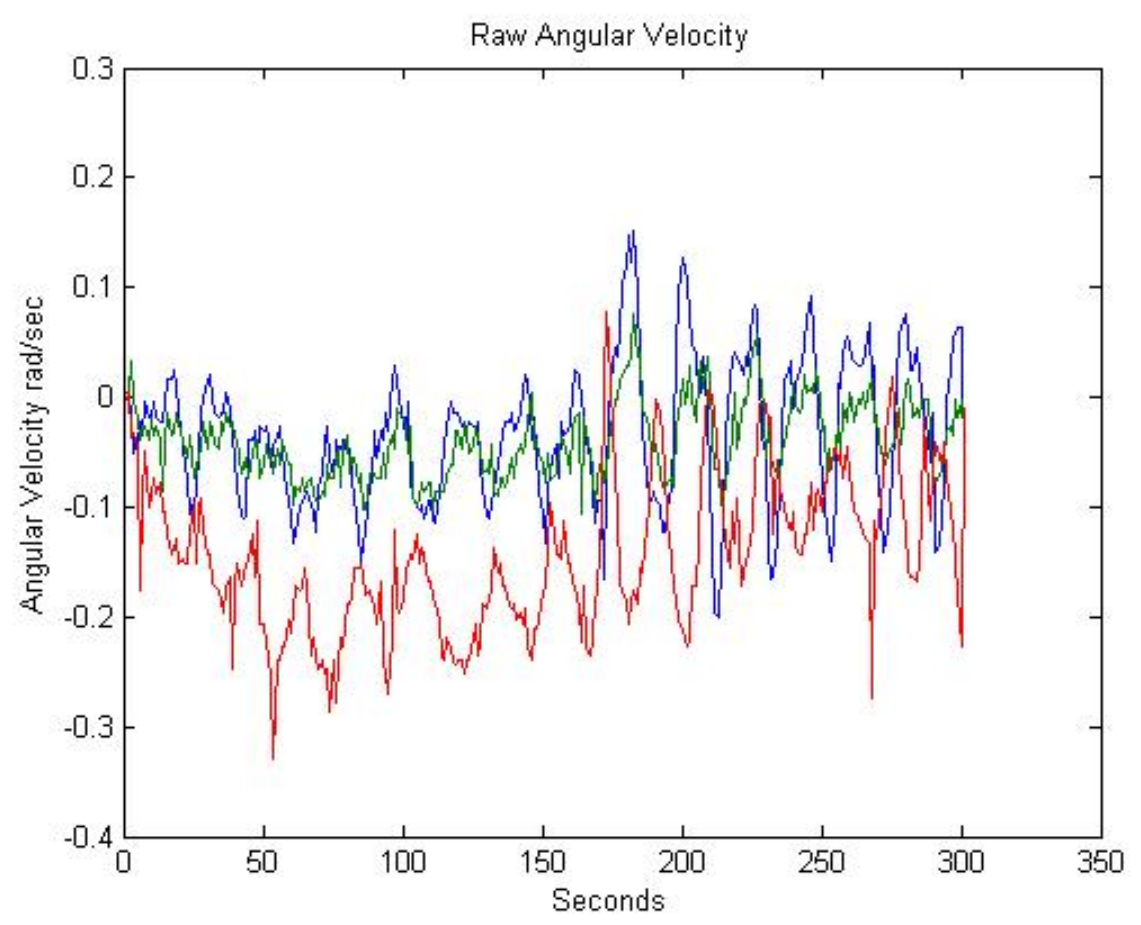

Figure 29: Experimental raw angular velocity data

Due to the noise experienced by the PRWP measurements for both the gyroscopes and the TACs, the readings from the ADC ports were averaged using a weighted averaging scheme. The averaging allowed the spikes in the data to reduced and sometimes ignored if the spikes in the data were small at a certain area of the data curve. Yet with this averaging, there is more error introduced into the readings because sometimes the averaging scheme would not follow the general line of the curve that the data exhibits. At times the averaged data would greatly reduce the intensity of a certain data range to the point where the resultant curve would ignore a great velocity that might have occurred at that time step because that great velocity might look like a noise spike instead of a possible velocity at that time. In short, the weighted averaging scheme could not differentiate between normal measurement noise and the true characteristic motion of the PRWP. In Figure 30, the raw data is compared to the averaged data of the angular velocity. As can be seen in the figure, the averaged data (blue line) is less erratic compared to the raw data 
(red line), but there is some shifting in data values due to the averaging of the data. This can be seen in wz graph at time step 50 seconds to about 75 seconds. Here the averaged curve is shifted to the right of the raw data. This shifting is due to the weighted averaging scheme. Yet with this averaging scheme there is an improvement to the data as opposed to not having the averaging, as can be seen in the results especially for the principle inertia about the z-axis
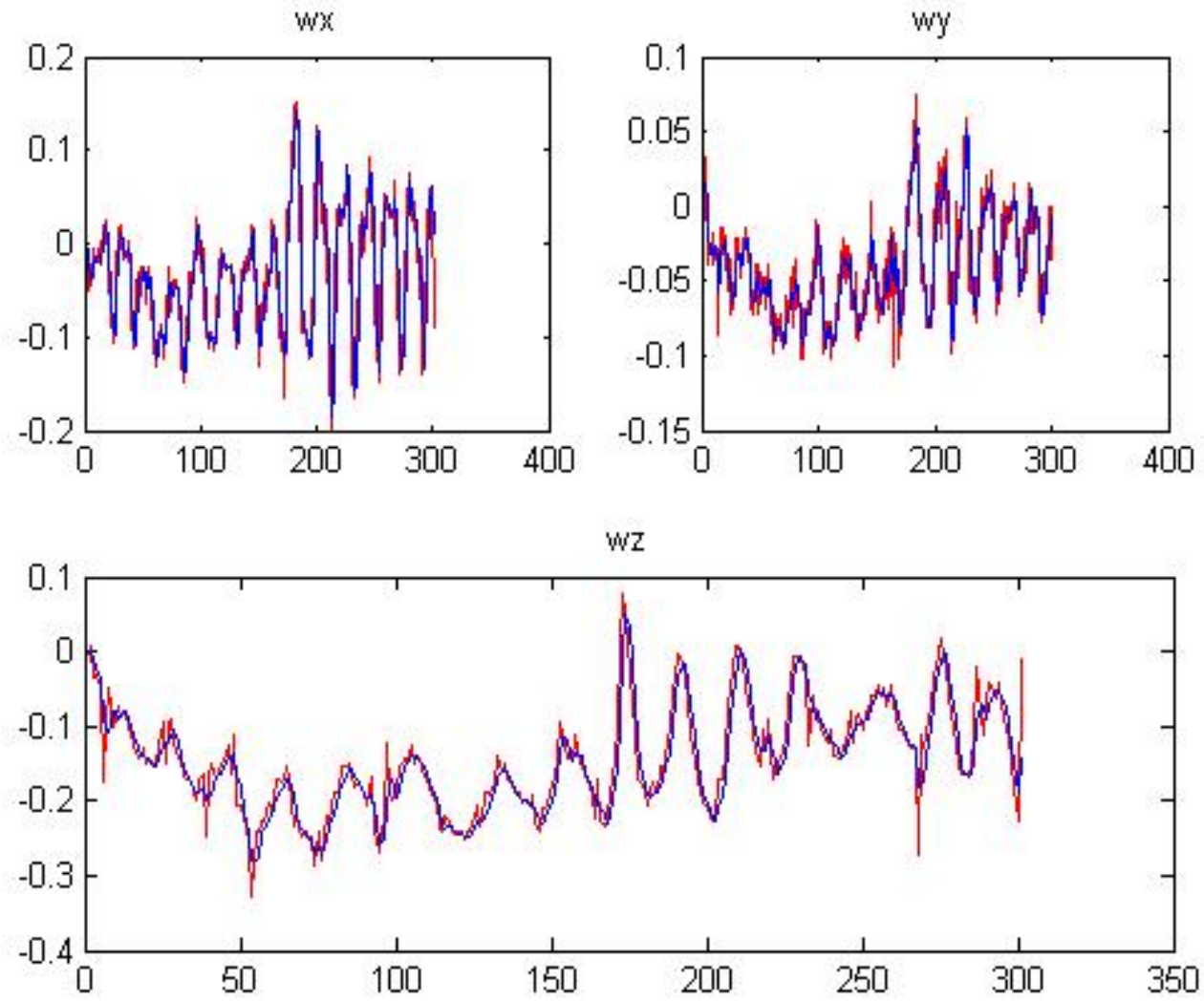

Figure 30: Angular velocity (Red - raw data; Blue - averaged data)

In Figure 31, the identified state using averaged data, column 2, is compared to the identified state using data that has not been averaged, column 3. The identified state using the non-averaged data is mostly negative except the principle inertia about the z-axis, but the identified state using the averaged data has the principle inertia about the $y$ - and z-axes positive, 
which is more realistic. The magnitude of the principle axes results is closer to the magnitude of the theoretical data obtained by Healy's theoretical simulation.

\begin{tabular}{|c|c|c|}
\hline Identified State & $\mathrm{x}$ exp w/averaging & $\mathrm{x} \exp$ w/o averaging \\
\hline $\mathrm{I}_{\mathrm{xx}}$ & -0.2094 & -0.0577 \\
\hline $\mathrm{I}_{\mathrm{xy}}$ & -0.1351 & -0.0529 \\
\hline $\mathrm{I}_{\mathrm{xz}}$ & -0.0750 & -0.0526 \\
\hline $\mathrm{I}_{\mathrm{yy}}$ & 0.1940 & -0.0014 \\
\hline $\mathrm{I}_{\mathrm{yz}}$ & -0.1434 & -0.0518 \\
\hline $\mathrm{I}_{\mathrm{zz}}$ & 0.5802 & 0.1227 \\
\hline
\end{tabular}

Figure 31: Identified state using averaged data vs. identified state without averaging

The averaging scheme is also very temperamental as well. By changing the length of the averaging vector has a great effect on the smoothness and accuracy of the averaging scheme. The length of the averaging vector determines how many data points are included into the weighted average. For example, if the averaging vector is 10 digits long, then the ten previous data points will be used to average the next data point. Also the gain on the averaging vector tends to move the averaged resultant curve higher or lower in overall value compared to the raw data. An example of the shift can be seen in Figure 32. The blue curves are the averaged data and the red curve is the raw data. There is a great discrepancy between the averaged data and the raw data as can be seen in the figure below. There is a difference of more than 1.5 radians per sec in difference between the raw and the averaged data. Therefore, the averaging scheme can introduce great error in the outcome of the experimental system identification process if not monitored. Due to the great ability of the averaging scheme to make the system identification process highly erred, the averaging scheme has to be designed specifically for the platform. Most of the recorded data of the system identification experimental runs have the averaging scheme optimized for that data by post-processing the data to ensure the averaged curve 
resembles the raw data. The diagram and the code for the post-processing algorithm can be seen in the appendix where the raw recorded data is run through the system identification algorithm with adjusted weights yielding a new identified state matrix. Figure 32 shows how the averaging scheme can affect the outcome of the system identification process, and how the averaging scheme could be adding to the error experienced by the solution of the experimental system identification process.
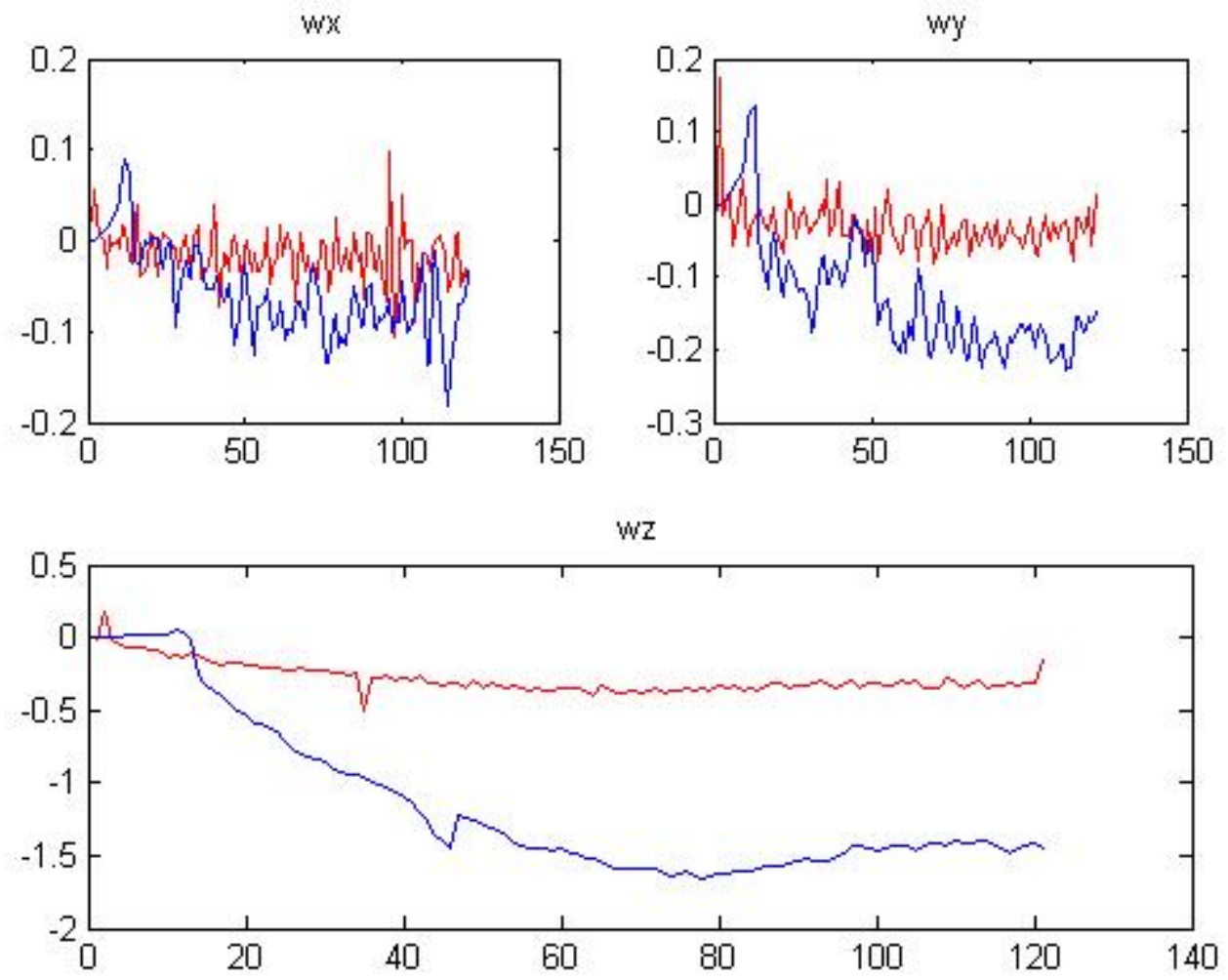

Figure 32: Angular velocity raw versus averaged using bad averaging scheme

Another contribution to the error can be attributed to the low bandwidth of the data retrieval from the PRWP. The gyroscopes and the TACs are read at a $1 \mathrm{~Hz}$ rate (one set of readings per second). This rate is relatively slow and it does not accurately map the motion that the spacecraft simulator experiences. To better characterize the PRWP, the rate or the readings 
from the system should be at least $10 \mathrm{~Hz}$, and this frequency is derived by Healy's simulation measurement rate. In that simulation, the angular velocity and acceleration readings are taken 10 times per second as opposed to the one second taken by the experimental system. The low bandwidth $(1 \mathrm{~Hz})$ can be shown to be a problem by applying the $1 \mathrm{~Hz}$ rate to Healy's simulation by only taking the reading for each integer second and no more. Through applying this process, the bandwidth can be proved to be a factor in the system identification error. In Figure 33, the high bandwidth case, at $10 \mathrm{~Hz}$ is compared to the low bandwidth case, at $1 \mathrm{~Hz}$, using the theoretical system identification simulation data. Due to the lowered bandwidth there is error seen from 14 percent up to 1200 percent, thus verifying that the low bandwidth can be attributed to some of the error experienced by the experimental system identification simulation.

\begin{tabular}{|c|c|c|c|}
\hline Identified State & x high BW & x low BW & \% difference \\
\hline $\mathrm{I}_{\mathrm{xx}}$ & 0.6499 & 0.1219 & -1200.5 \\
\hline $\mathrm{I}_{\mathrm{xy}}$ & 0.000458 & -0.1421 & 80.1 \\
\hline $\mathrm{I}_{\mathrm{xz}}$ & -0.000540 & -0.0213 & 50.7 \\
\hline $\mathrm{I}_{\mathrm{yy}}$ & 0.6001 & 0.4790 & -33.9 \\
\hline $\mathrm{I}_{\mathrm{yz}}$ & 0.0015 & -0.0402 & 78.5 \\
\hline $\mathrm{I}_{\mathrm{zz}}$ & 0.5598 & 0.7508 & 41.3 \\
\hline $\mathrm{mgr}_{\mathrm{x}}$ & 0.0405 & 0.0485 & 14.1 \\
\hline $\mathrm{mgr}_{\mathrm{y}}$ & -0.0981 & -0.0981 & -109.7 \\
\hline $\mathrm{mgr}_{\mathrm{z}}$ & 1.3872 & 0.4309 & -198.8 \\
\hline
\end{tabular}

Figure 33: Theoretical high bandwidth versus low bandwidth identified matrices

Finally, taking the derivative of the measured data to extrapolate the angular accelerations experienced by the PRWP and angular accelerations of the wheels can attribute to the error. The derivative that is taken on the TAC readings to get the angular accelerations of the wheels could add great error because the angular accelerations of the wheels are the largest component of the torque experienced by the platform, and if the torques due to the wheel accelerations have error introduced into their measurements, the overall solution will be affected. 


\section{Chapter 6: Conclusion}

The algorithm designed to handle the TCP/IP communication with the "ground" computer and the gumstix as well as data acquisition from the robostix and wheel commands from the "ground" computer to the robostix was designed and tested successfully. The algorithm acted as an active server listening for client connection request from MATLAB/SIMULINK on the "ground" computer. The server can run at $1 \mathrm{~Hz}$ and can send one set of wheel commands and read 1 set of gyroscope and TAC data per second. However, if the server is run any faster than $1 \mathrm{~Hz}$, it will crash due to the inherit lag of system calls needed to communicate from the robostix to gumstix and vice versa via the i2c communication program.

The experimental simulation process was also verified using the state and torque data from the theoretical system identification simulation. This data was inputted into the experimental system identification simulation algorithm and the resulting identified state matrix was compared to the theoretical identified state matrix. The error between the two theoretical and experimental state matrices was small and was predominately due to derivative and integral error in the experimental simulation algorithm. Another cause of this error was due to the fact that for the first 0.1 seconds in the theoretical simulation there were seven time steps and after that, there was 1 time step per 0.1 seconds. In the experimental simulation, there was 1 time step per 0.1 seconds for the entire simulation. This discrepancy causes an error in the derivatives and integrals taken for the first 0.2 seconds.

The experimental system identified matrix has large error when compared to the theoretical simulation's identified state matrix. The convergence history of the experimental simulation differs greatly for principle inertias about the y-and z-axes of the theoretical simulation, and the converged values differ greatly as well. Yet, the principle inertia about the z- 
axis is much closer to the theoretical value compared to the other inertia terms because there is more movement about the z-axis to damp out the noise experienced by the gyroscope readings.

There were several factors that attributed to the errors found in the experimental simulation solution. One factor was the noise found from fluctuating voltages that cause ADC values to be erratic and cause spikes in the recorded data. Also, the weighted averaging scheme employed to smooth out the spikes in the data added to the error experienced by the system by cutting down the intensity of the recorded values and shifting them as well. Also the weighted averaging scheme cannot differentiate between noise and actual PRWP movement so actual simulator movement might be ignored. Furthermore, changing the averaging vector for the weighted averaging scheme also affects to solution drastically making it difficult to determine what the appropriate solution should be. The low bandwidth, $1 \mathrm{~Hz}$, attributed to the third factor or error in the experimental system identification simulation process. The low bandwidth causes data to not be rich enough to identify the state. Also, due to the low bandwidth, the sensor data cannot accurately record the PRWP's movement. Finally, the last factor of error is attributed to derivative and integral error. 


\section{Chapter 7: Future Work and Recommendations}

Due to the latency issue by using system calls to communicate from the gumstix server to the robostix via i2c, some of the data handling could be hard coded onto the robostix memory allowing the robostix to function at it highest speed without going through i2c to send and receive data. The data handling would include the reading of analog to digital ports and writing to the pulse-width modulation ports. For the other portion of code on the sever that sets up the TCP/IP communication and serial communication, these portions of code will be on the gumstix memory due to the fact that the Bluetooth module and connection to the serial port are native to the gumstix. Also the terminal access for the "ground" computer to start the server will go through the gumstix as well. 


\section{List of References}

1. Carson, Mittelsteadt O. "Results on the Development of a Four-Wheel Pyramidal Reaction Wheel Platform." Thesis.

2. "Gumstix." Gumstix: Dream, Design, Deliver. Ed. Gumstix Inc. Gumstix. Web. <www.gumstix.com>.

3. Hashimot, Seiji, Tomonori Ogawa, Shuichi Adachi, Anzhong Tan, and Gou Miyamori. "System Identification Experiments on a Large-scale Unmanned Helicopter for Autonomous Flight." IEEE International Conference on Control Applications, 25-27 Sept. 2000, Anchorage, Alaska, USA.

4. Healy, Patrick B. "Mass Property System Identification of a Spacecraft Simulator." Thesis.

5. "I2C-Bus: What's That?" I2C-Bus: What's That? Web. <http://www.i2c-bus.org/>.

6. Juang, Jer-Nan. Applied System Identification. Upper Saddle River: Prentice Hall PTR, 1993.

7. Kim, Jae Jun, and Brij N. Agrawal. "Automatic Mass Balancing of Air-Bearing-Based Three-Axis Rotational Spacecraft Simulator." JOURNAL OF GUIDANCE, CONTROL, AND DYNAMICS May-June 200932.3 (2009): 1005-017. Print.

8. Logan, Jeff. Control of Pyramidal Reaction Wheel Platform. Thesis, 2008.

9. Saile, Christopher A. Center of Mass Fine Tuning System for the Cal Poly Spacecraft Simulator. 
10. Schwartz, Jana L., and Christopher D. Hall. "Comparison of System Identification Techniques for a Spherical Air-Bearing Spacecraft Simulator." AAS/AIAA Astrodynamics Specialist Conference, 3-7 Aug. 2003. 


\section{Appendix A: Simulation Procedure}

This section will diagram the steps that are necessary to perform the system identification process on the PRWP.

\section{Power On}

In order to run the PRWP, there is a specific start-up sequence necessary for the PRWP to run effectively and properly. The first step that needs to take place is the plug in the battery power to the mainboard. The user has to make sure the DC adapter from the mainboard is also plugged in the DC port of the robostix. This specific adapter provides the power for the robostix and the gumstix. Once the battery and the DC adapter are plugged in, then the toggle switch for the power router on the mainboard should be switched to the on position.

The next battery pack to plug in is the two large 12 volt lead-acid batteries that will power the reaction wheels and the CMFBS. The two batteries are connected serially bringing their output voltage near 24 volts necessary to run the reaction wheels and the FAULHABER microcontrollers controlling the fine-balancing motors. Once the battery power adapter is plugged into the PRWP adapter located on the middle shelf of the PRWP, the large red toggle switch should be toggled to the right to turn on the power to the entire PRWP. If the power is on, a yellow light on the serial connector mounted on the shelf above the mainboard should be turned on. 


\section{Programs}

\section{$\underline{\text { PRWP gumstix }}$}

When the power is turned on to the robostix and gumstix via the mainboard, the gumstix and robostix will boot. The ground computer acquires the connection automatically and the connection icon in the taskbar of the ground computer will indicate that the gumstix is connected. Once this happens, the user can open the PuTTY client, which will serve as the terminal client for the gumstix through the wireless connection. Once logged on, the user will type in "uisp". This will start the i2c-io program and commands the gumstix to locate the robostix. Then the user types in "cd /usr", which will change the directory to the user directory that holds all the server programs needed for communication with the ground computer. Once in the directory, the user can type "./gumstixServ", which will start the gumstix server program. This program, as described before, will allow MATLAB, on the ground computer, to connect as a client to the gumstix. If errors are displayed, type CTRL-c and retype "./gumstixServ". The gumstix has a tendency to act finicky. If the problem persists, continue retyping the CTRL-c and typing in the gumstixServ command two or three more times, until no errors come up. If the problem still persists, then unplug the power to the robostix and check whether the power going into the robostix is above 4.5 volts.

Once the server program is activated, the program will prompt the user to type a 0 to center the masses or type a 1 to skip the step. The user will enter the desired command number and then press the enter key. If the user chooses to center the masses, the user must wait till the masses reach their extreme on the track. The program will ask the user to press 1 when the masses reach the extreme. Once that happens, the user will type the number 1 and then press 
enter. After the centering of the fine-balancing counterweights, or after skipping this step, the server is now ready to accept client connections from MATLAB.

\section{$\underline{\text { Ground Computer }}$}

For the ground computer, open MATLAB and go to the "PRWP Code" directory, and then navigate to the "System" directory and open the systemIDStart.m file. This program, as described before will run the system identification process. It will run all the necessary simulation models. It will run the systemIDReal simulation model file that will command the reaction wheels with amplified discrete sine signals, and it will read sensor data from the TCP/IP socket. This program will compute the system identified matrix and extrapolate the distance the $\mathrm{CM}$ is from the pivot point. The function will then send the appropriate distance to move the counterweights that are apart of the CMFBS to counteract the imbalance in mass on the PRWP. 


\section{Appendix B: C and MATLAB Code and SIMULINK Model for}

\section{Experimental System Identification}

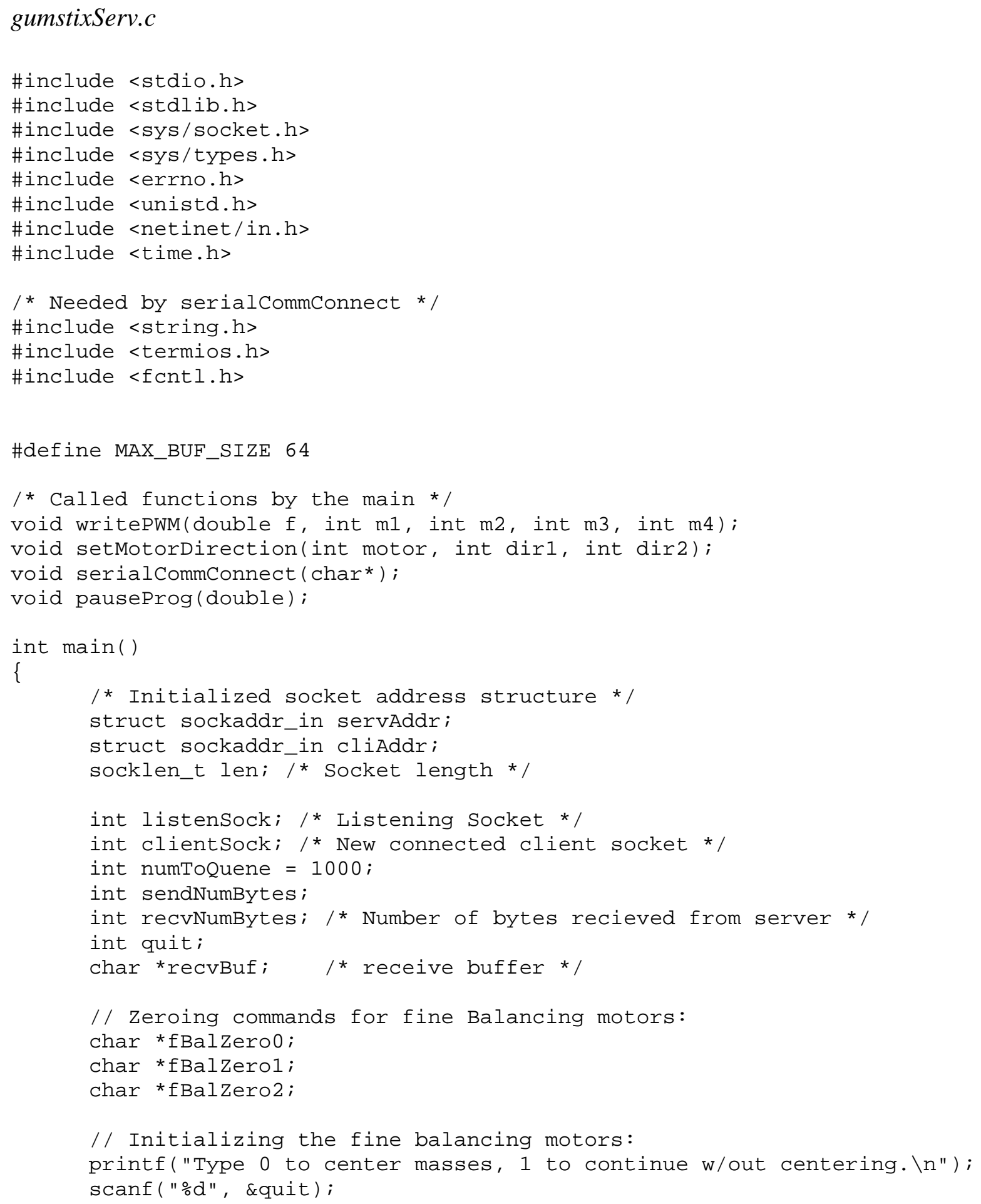




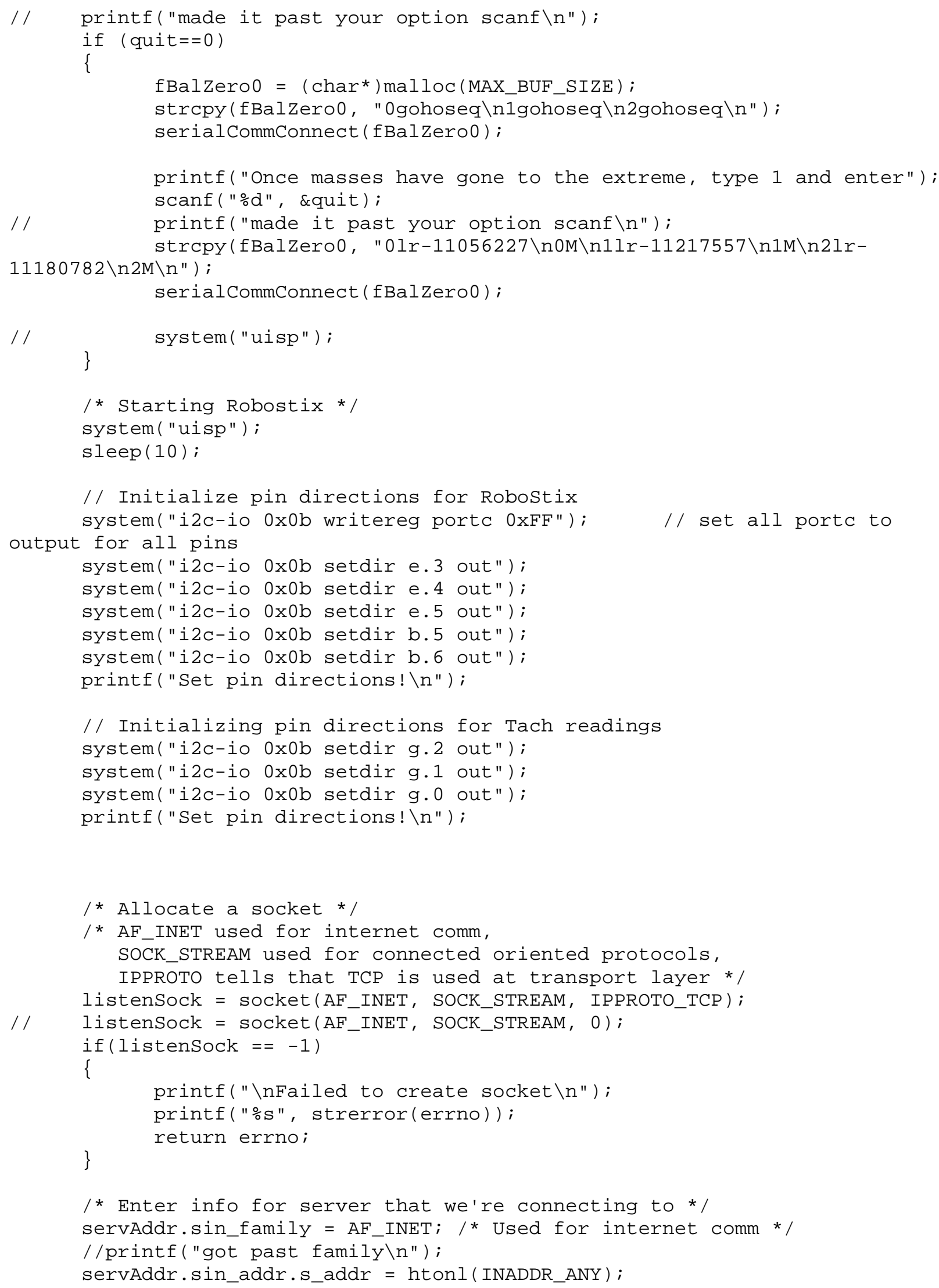




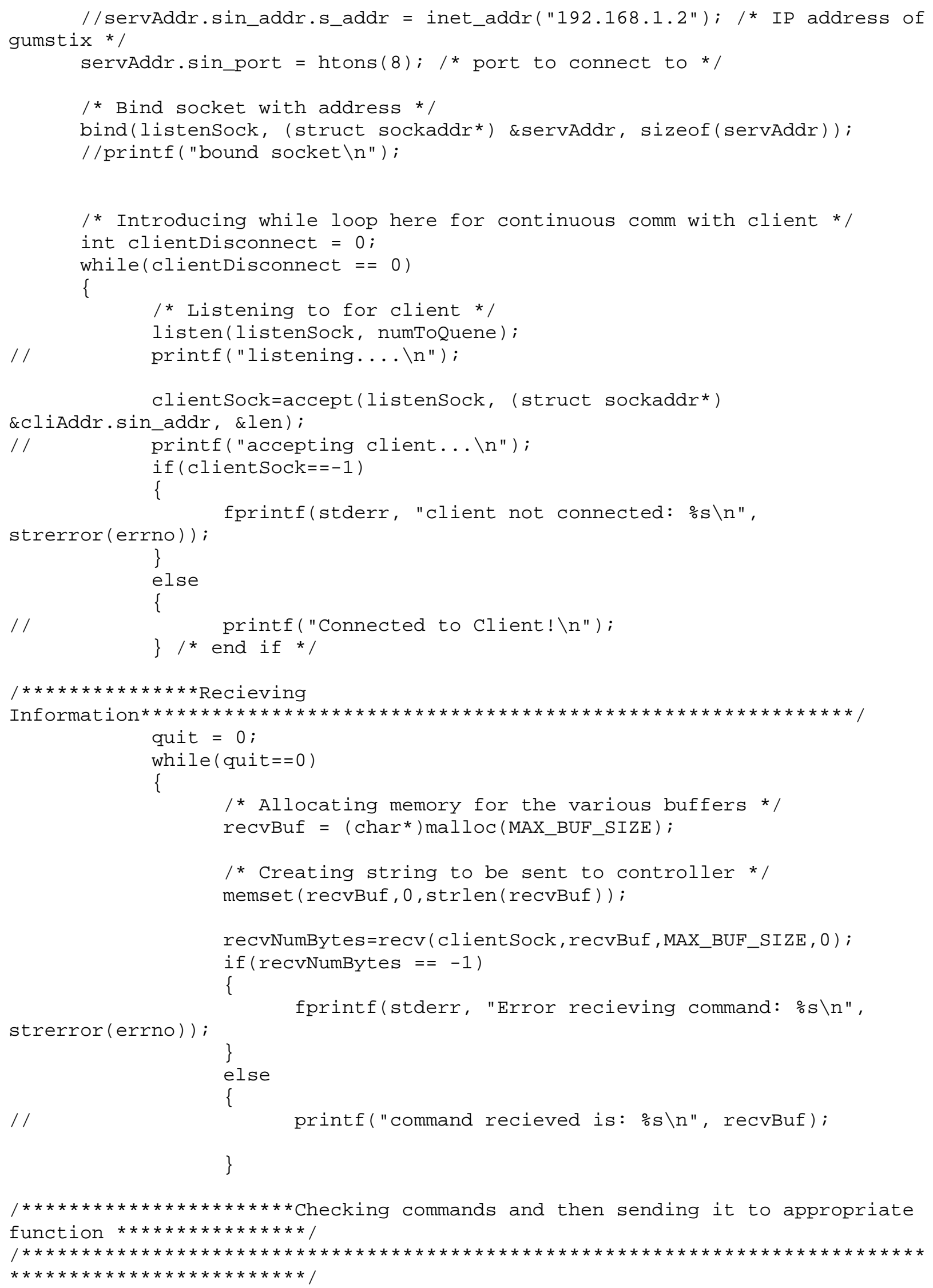




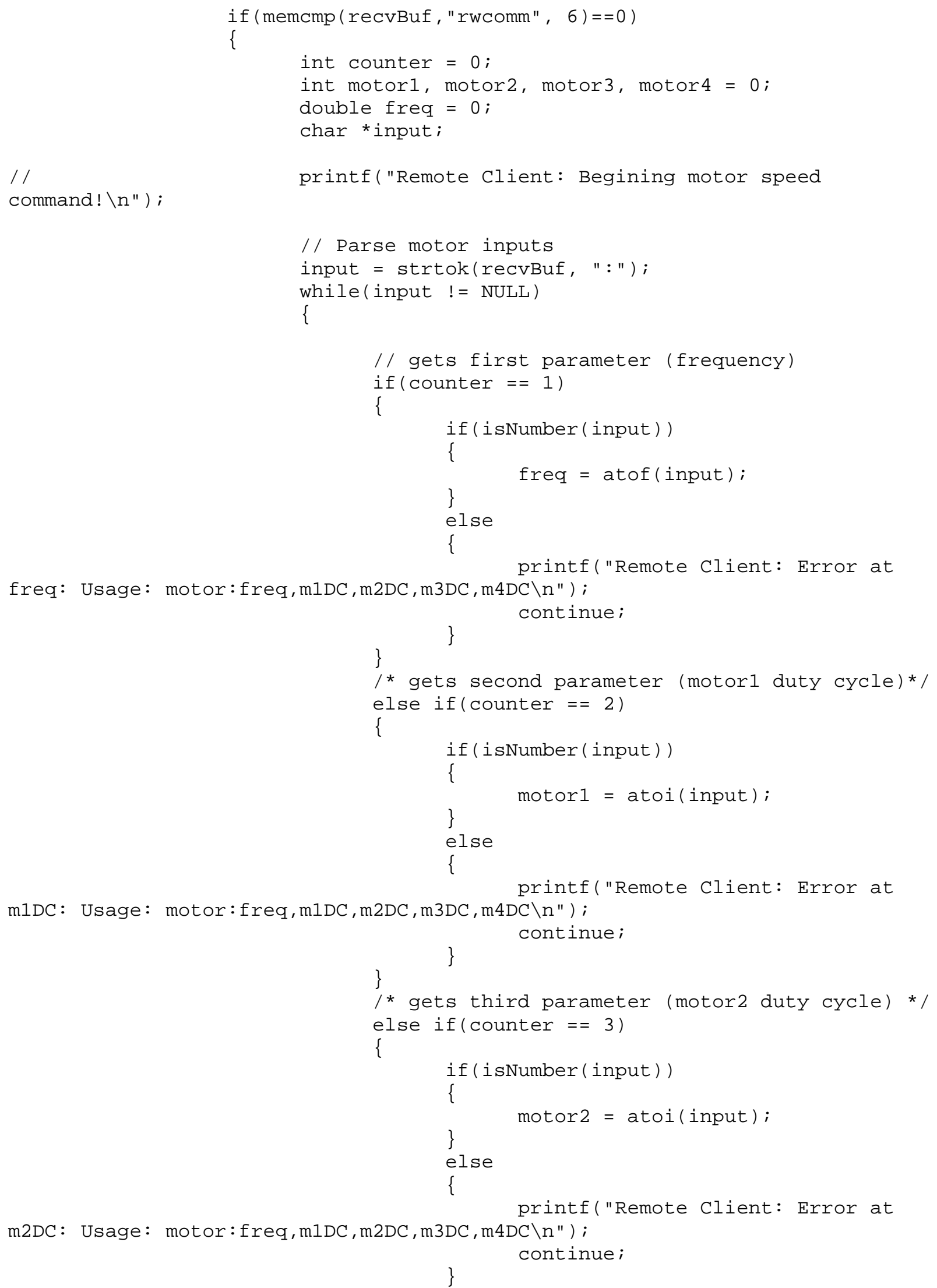


\}

/* gets fourth parameter (motor3 duty cycle) * / else if(counter $==4$ )

\{

if (isNumber (input))

\{

\}

motor3 = atoi (input);

else

\{

printf("Remote Client: Error at

m3DC: Usage: motor:freq, m1DC,m2DC, m3DC,m4DC \n") ;

continue;

\}

\}

/* gets fifth parameter (motor4 duty cycle) */

else if (counter $==5$ )

\{

if (isNumber (input))

\{

motor4 = atoi (input);

\}

else

\{

printf("Remote Client: Error at

m4DC: Usage: motor: freq, m1DC, m2DC, m3DC, m4DC \n") ;

continue;

\}

\}

parameter

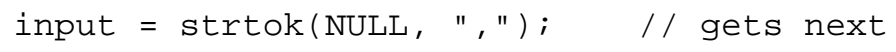




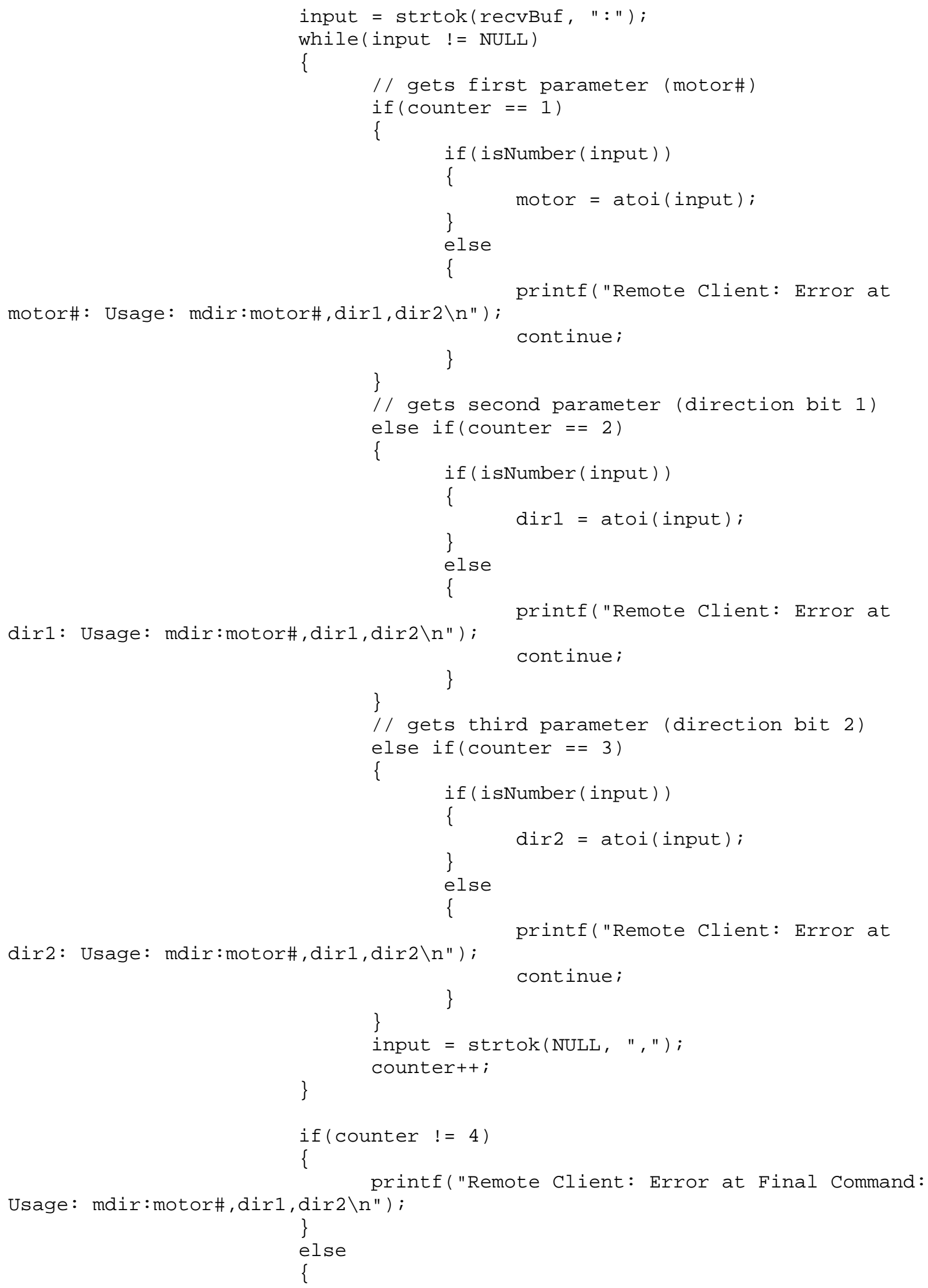




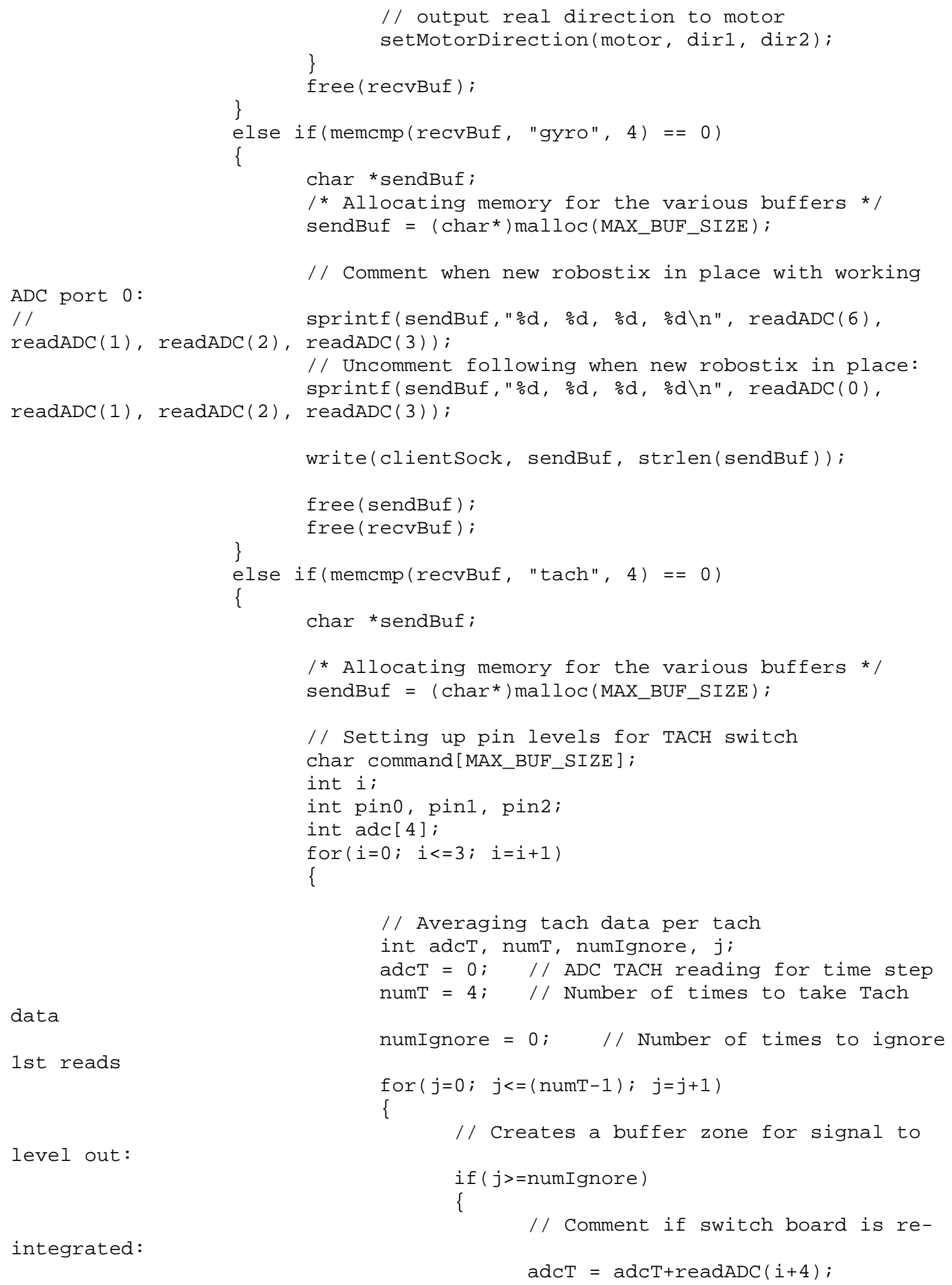




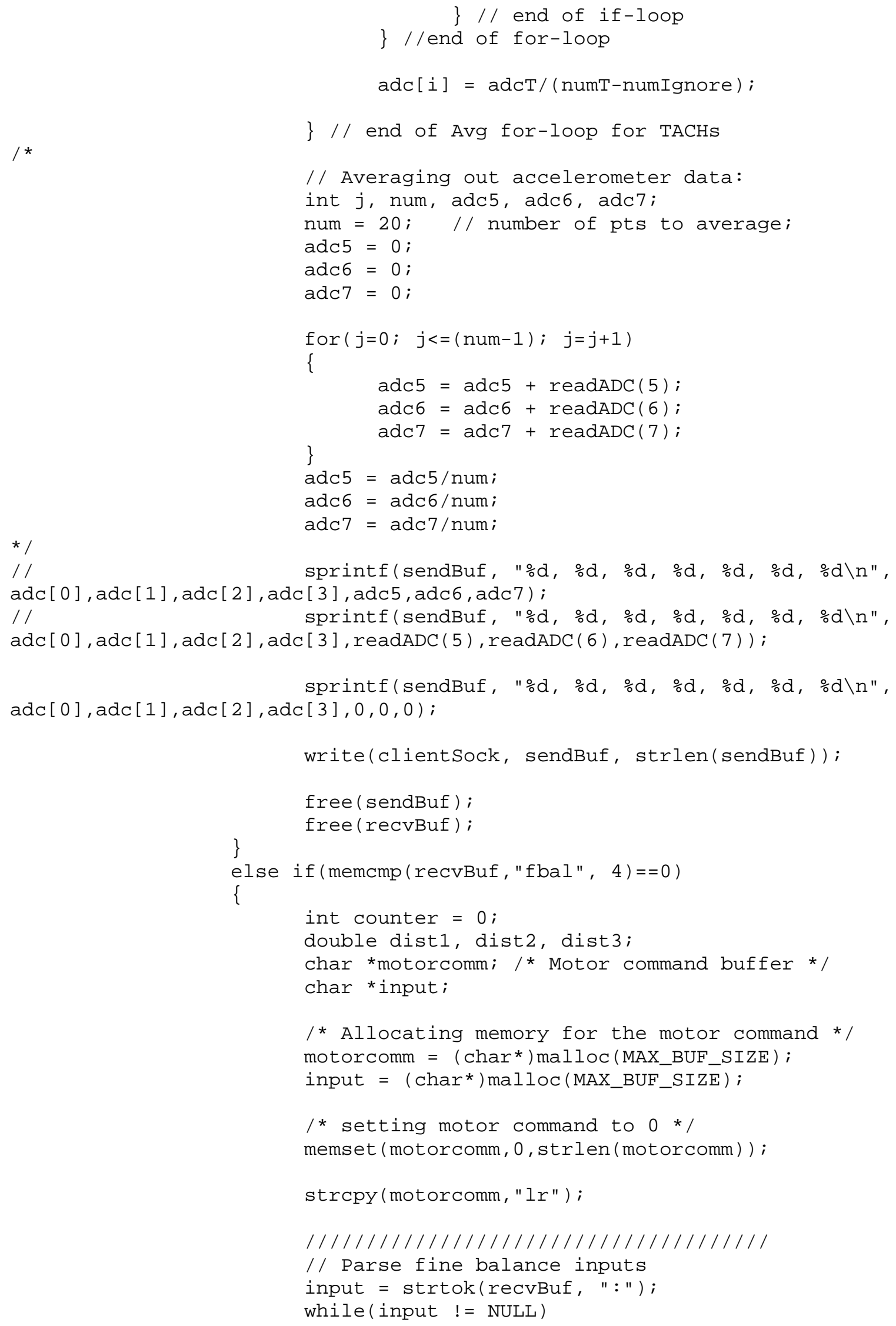


motor:

motor:

motor:

/ / gets first distance

if (counter $==1$ )

\{

if (isNumber (input))

\{

dist $1=$ atof (input);

// converting from $m$ to ticks on

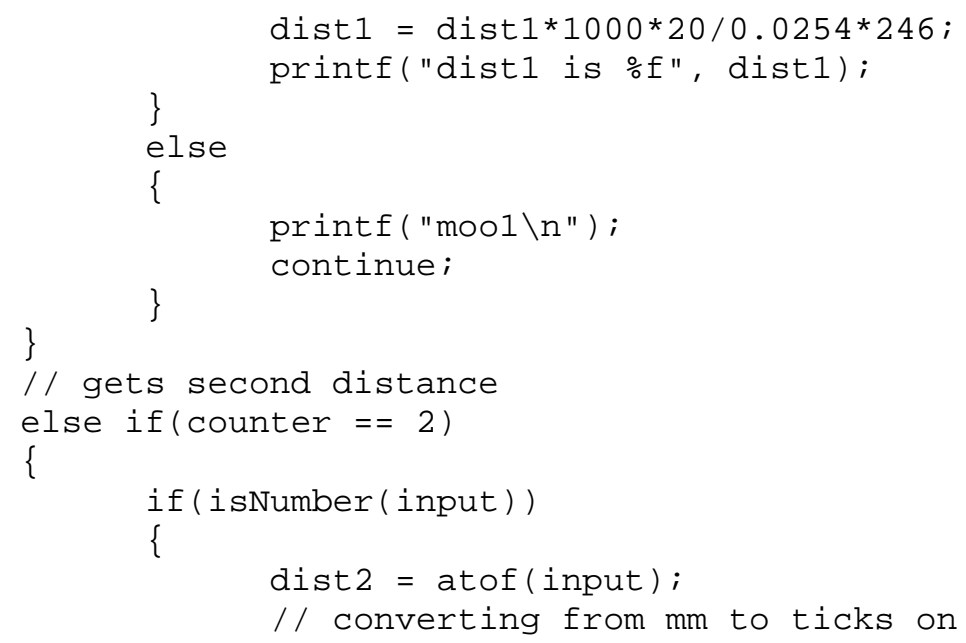

parameter

counter++;

printf("input is $=\circ s \backslash n "$, input); 


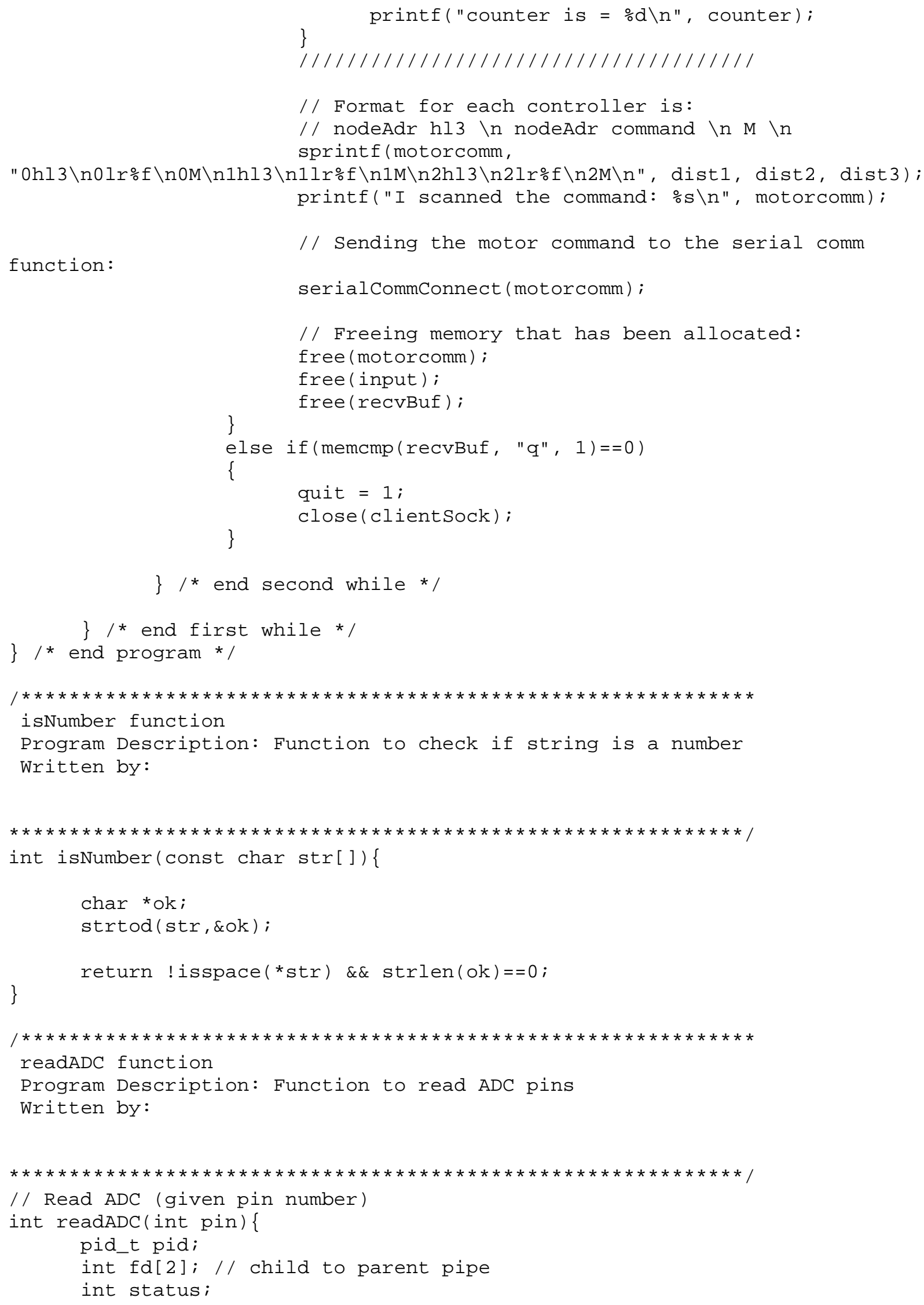




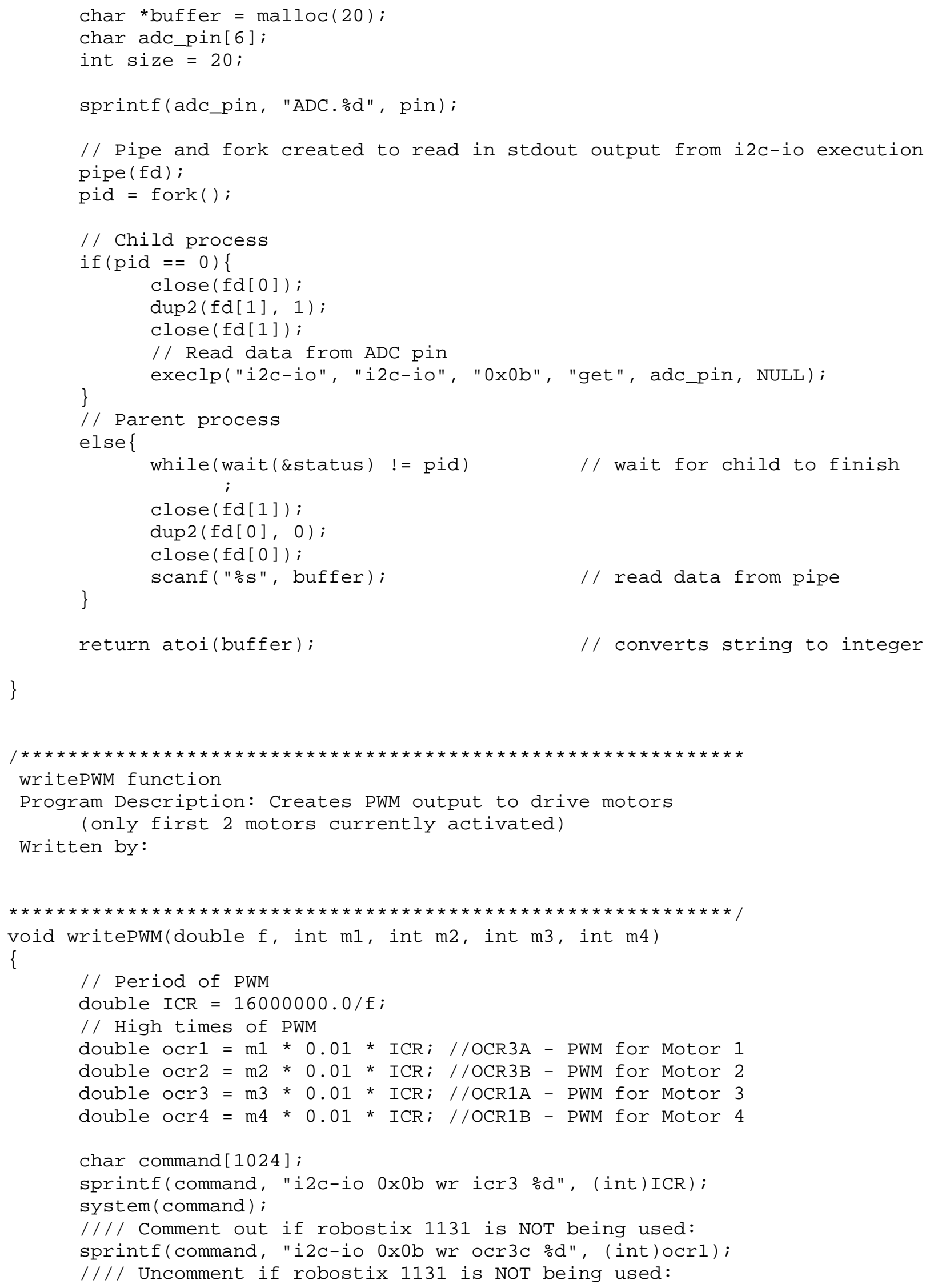




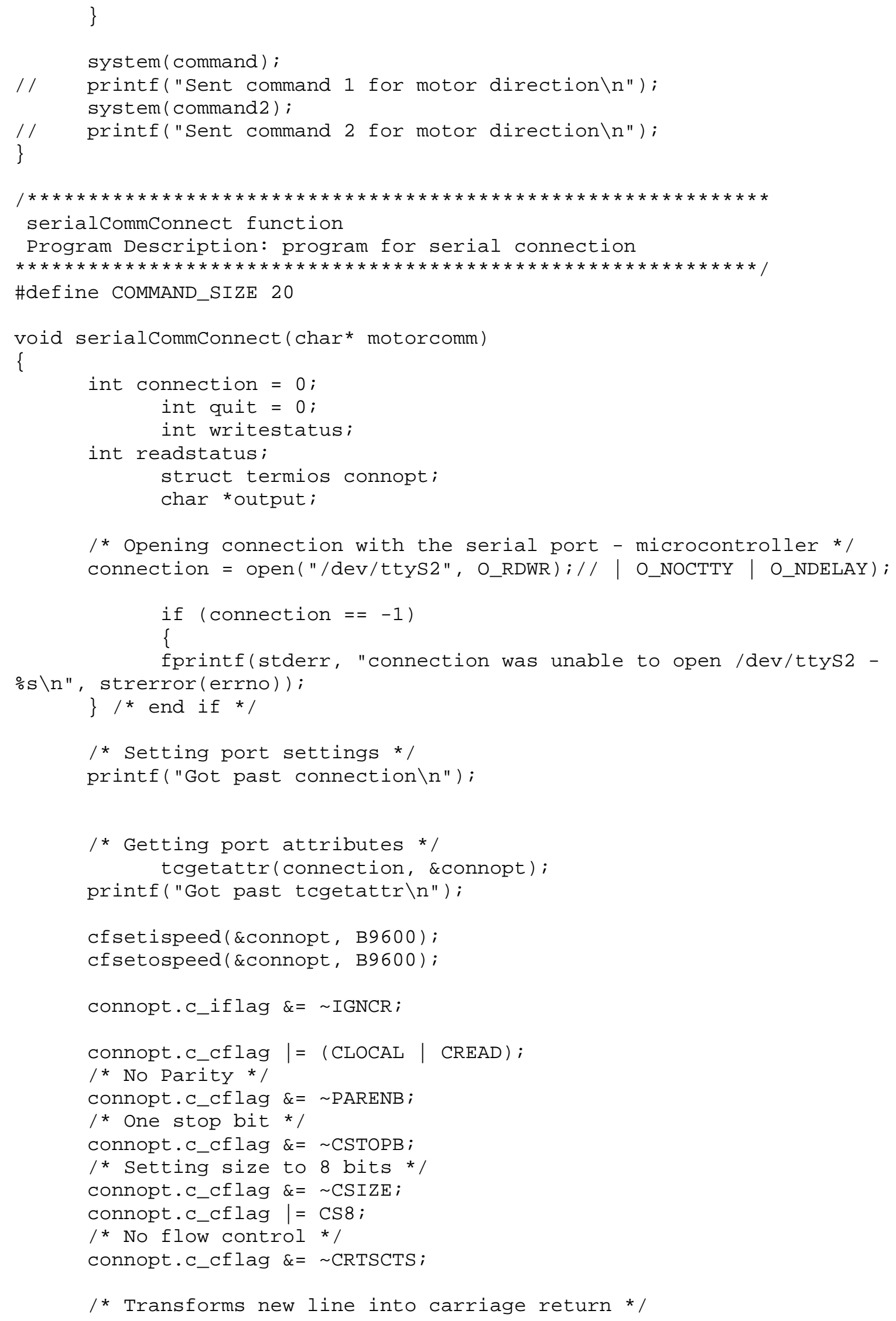




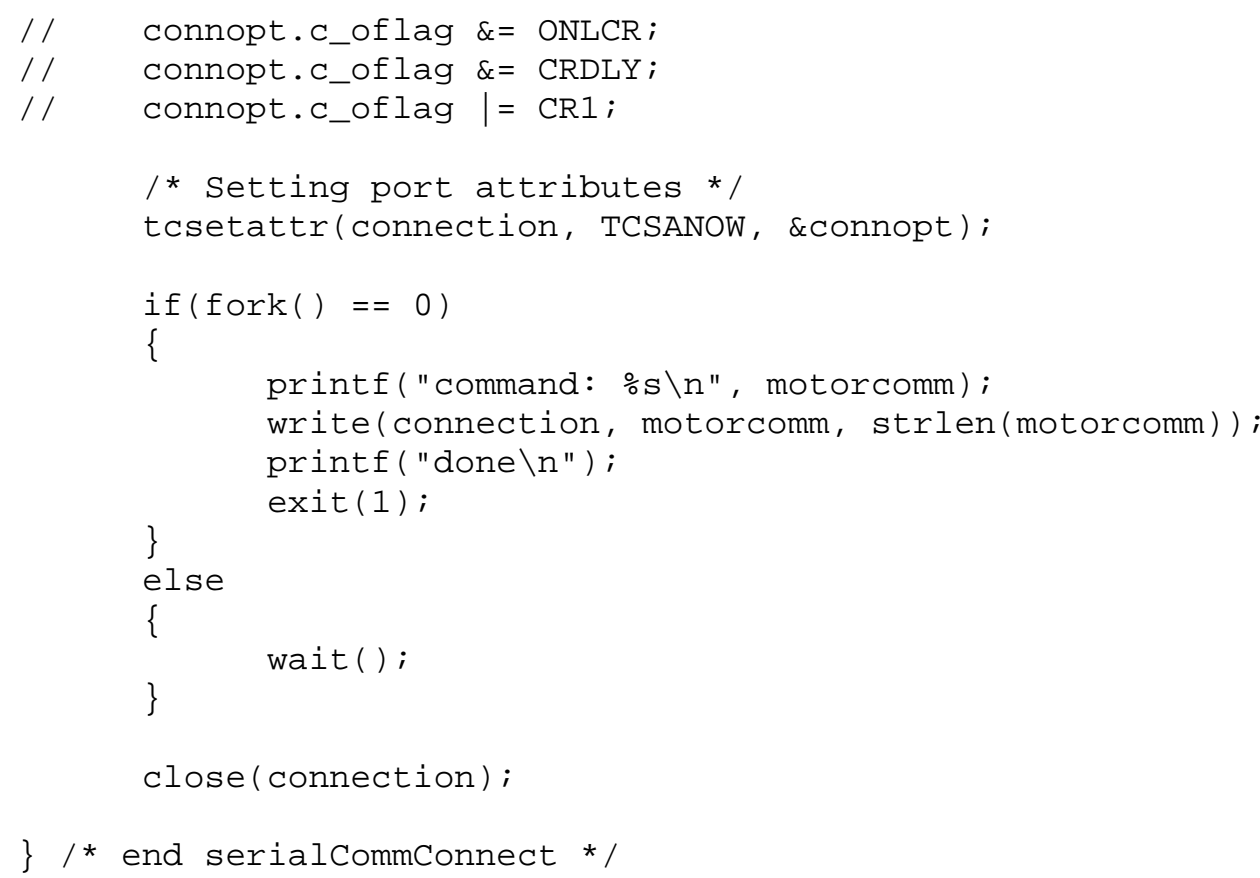

\section{gumstixSend.m}

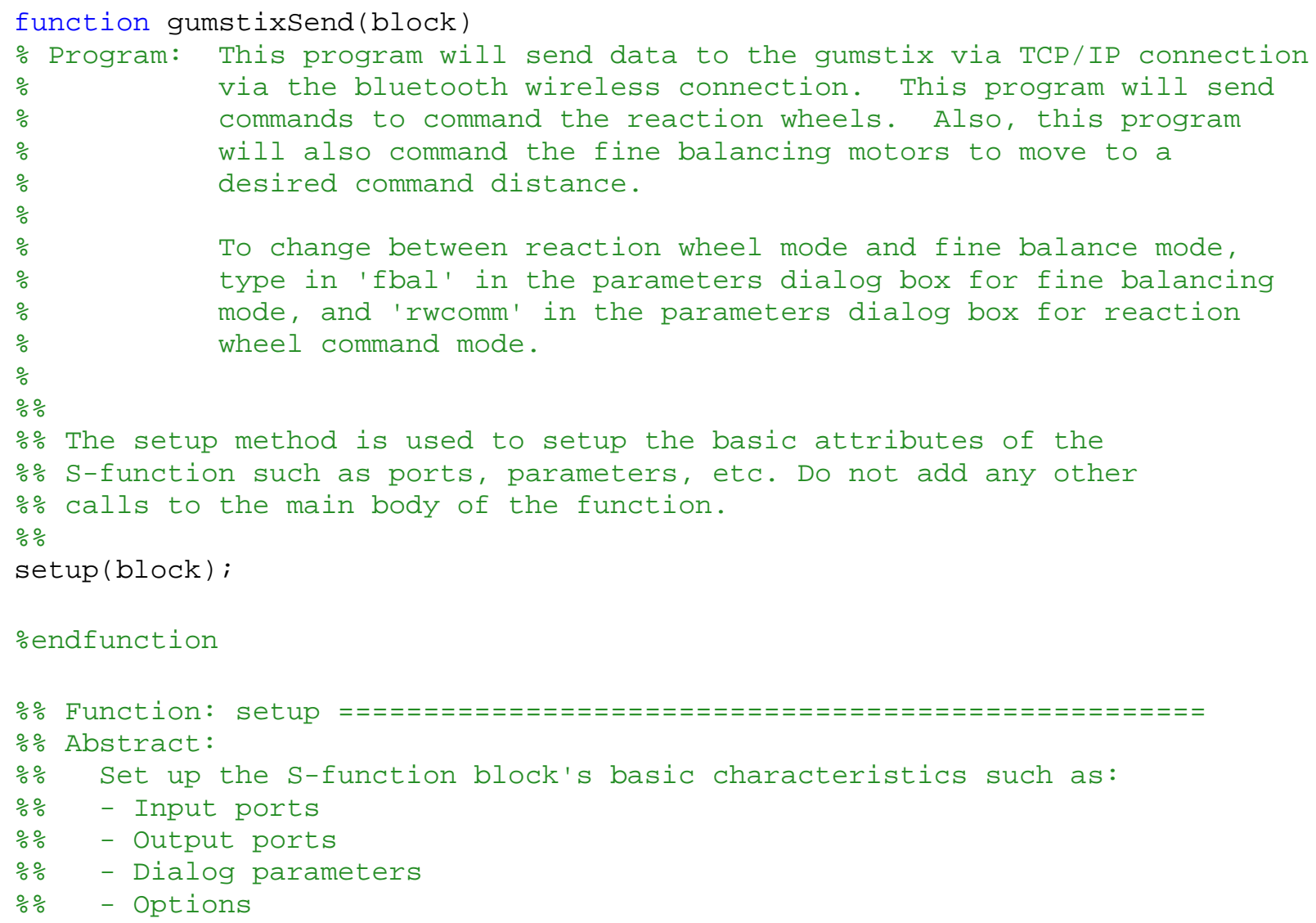




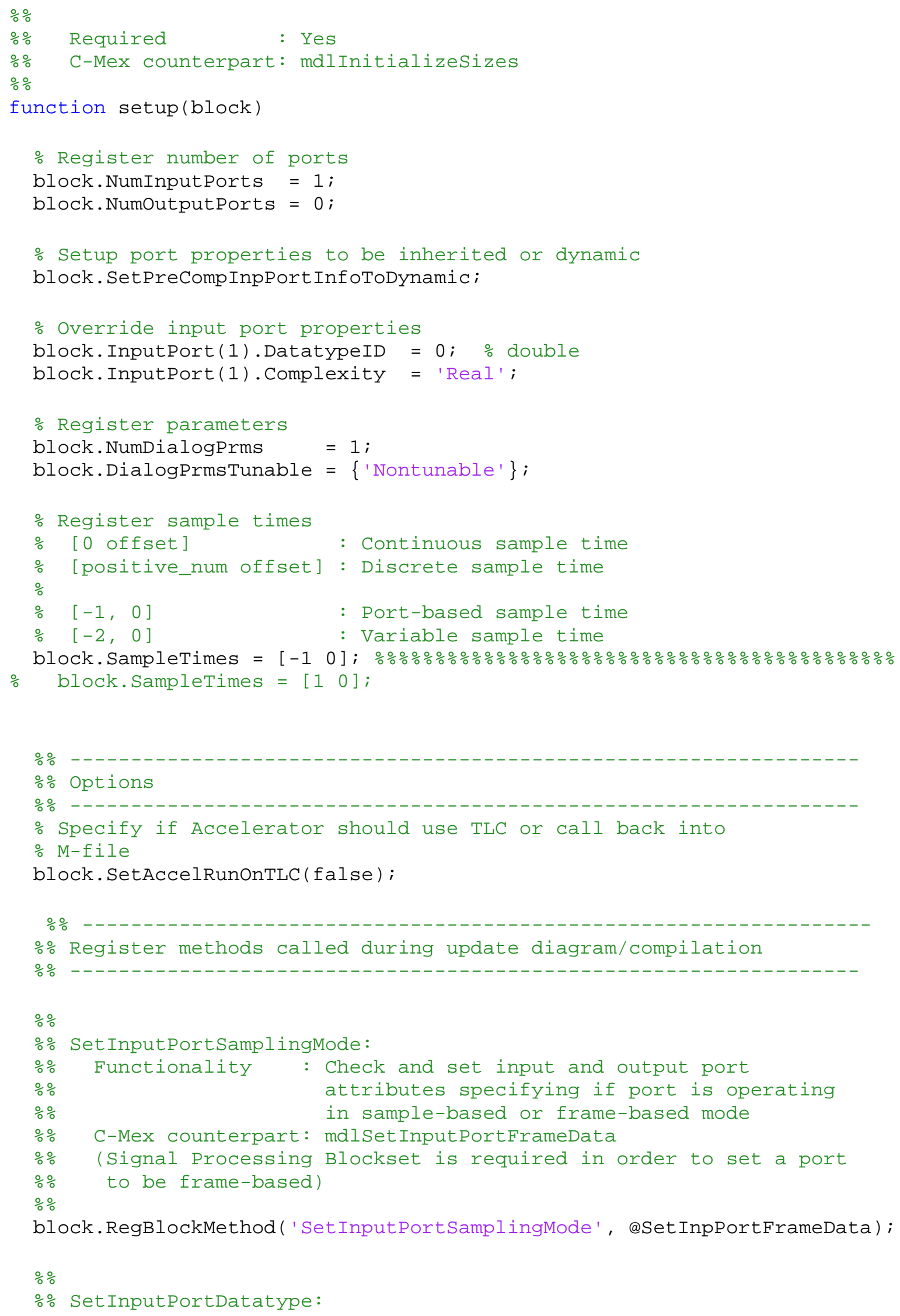




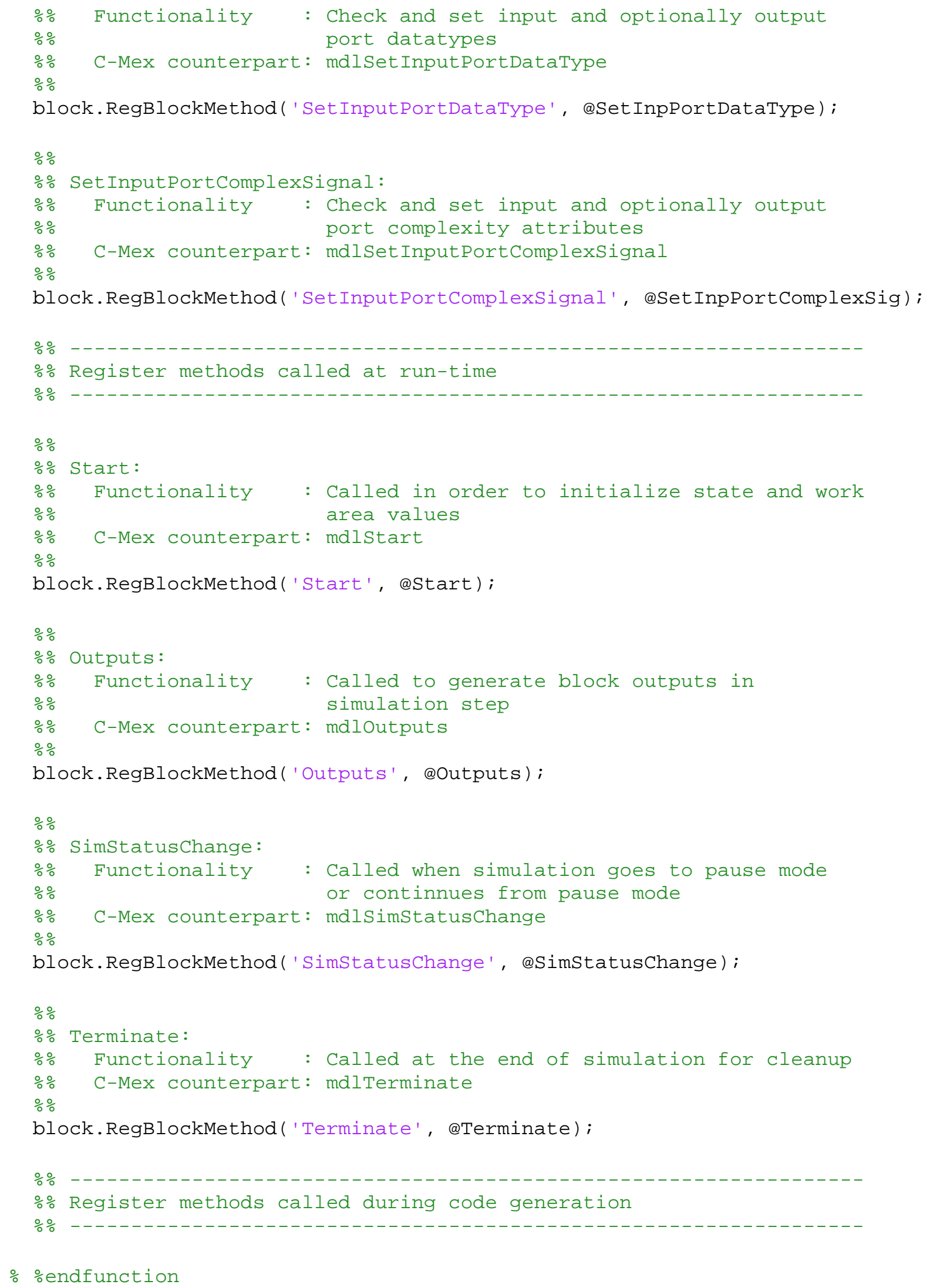




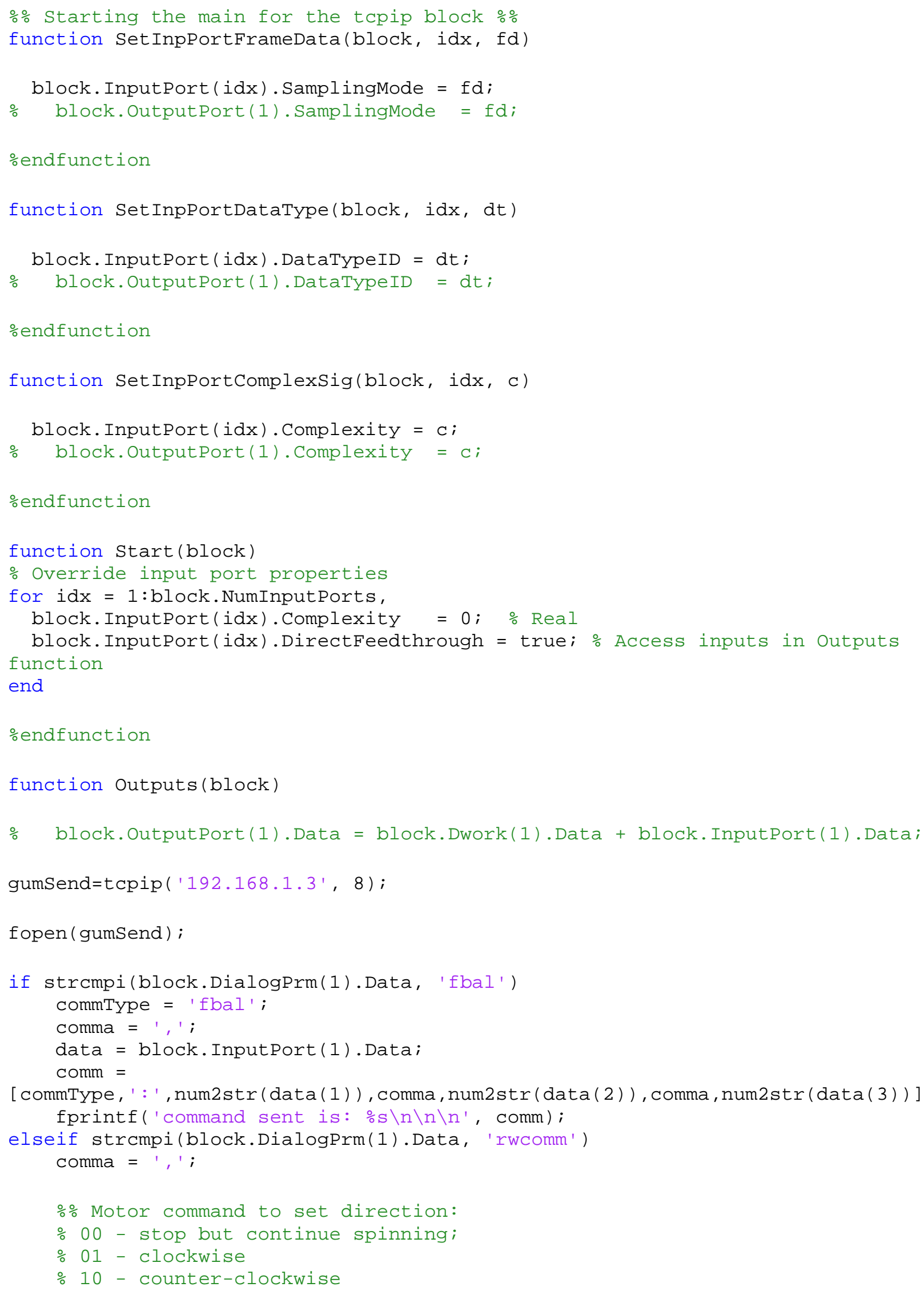




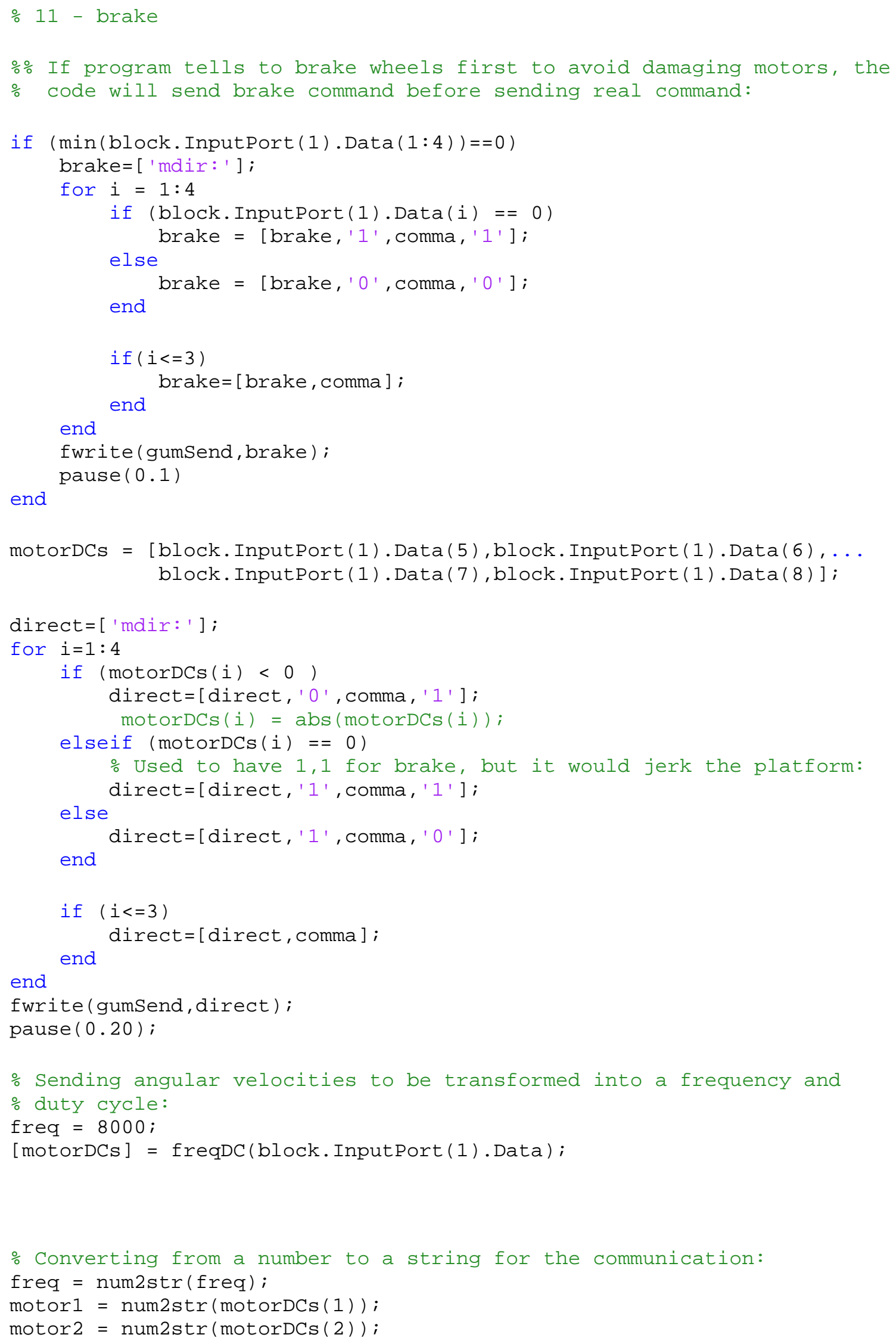




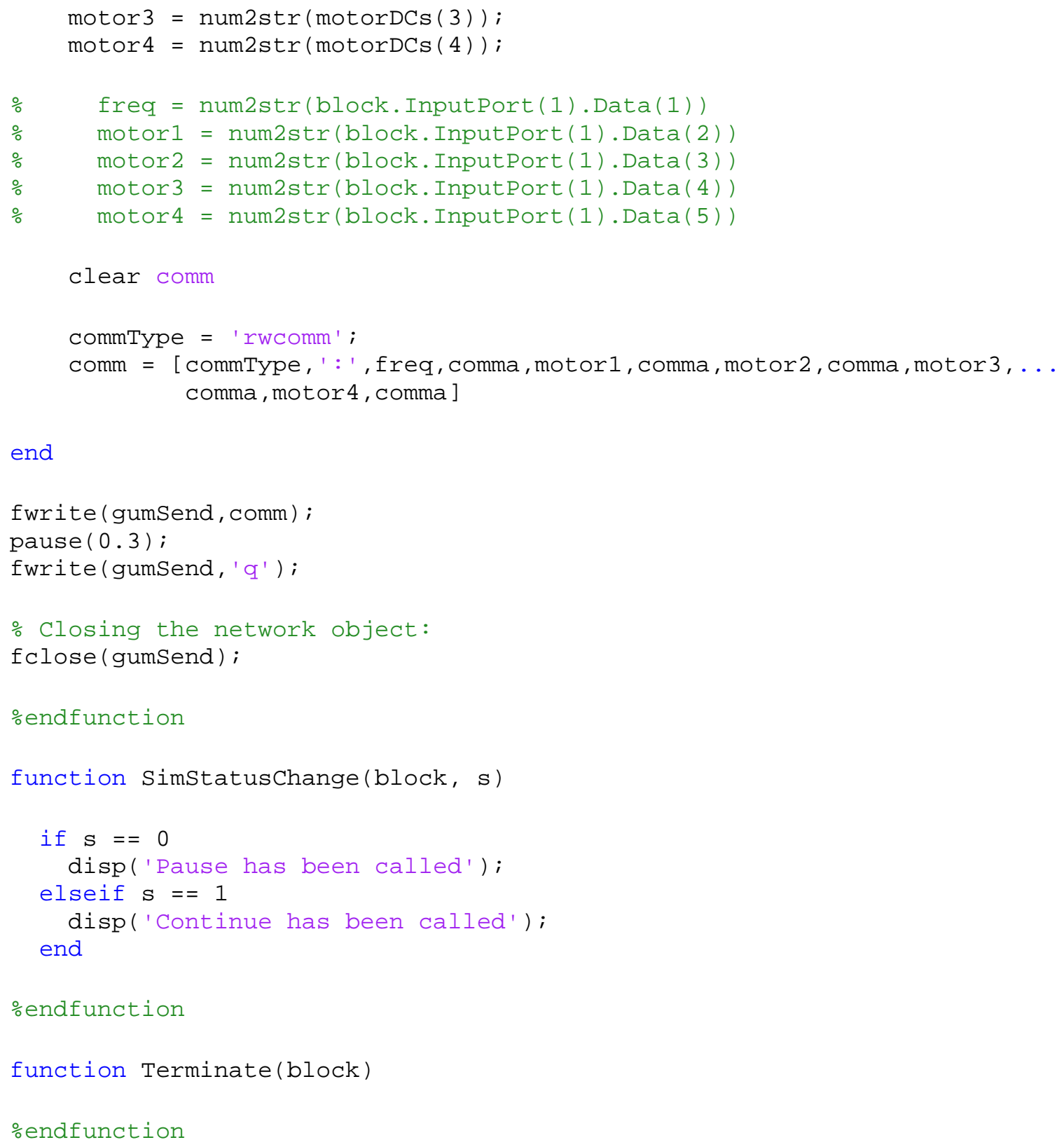


\% calls to the main body of the function.

setup (block);

SetoutputPortDataType (block);

SetoutputPortDims (block);

\%endfunction

$\div$ Function: setup $===========================================$

\% Set up the s-function block's basic characteristics such as:

\% - Output ports

\% - Dialog parameters

응 - Options

$\div$

function setup (block)

\% Parameters:

Register number of ports

block. NumOutputPorts $=1$;

block. NumDialogPrms = 1;

block.DialogPrmstunable $=\{$ 'Nontunable' $\}$;

\% Register sample times

block. Sampletimes $=\left[\begin{array}{ll}-1 & 0\end{array}\right]$;

- Specify if Accelerator should use TLC or call back into

M-file

block. SetAccelRunontLC (false);

\% block. SetSimViewingDevice(true); $\%$ no TLC required

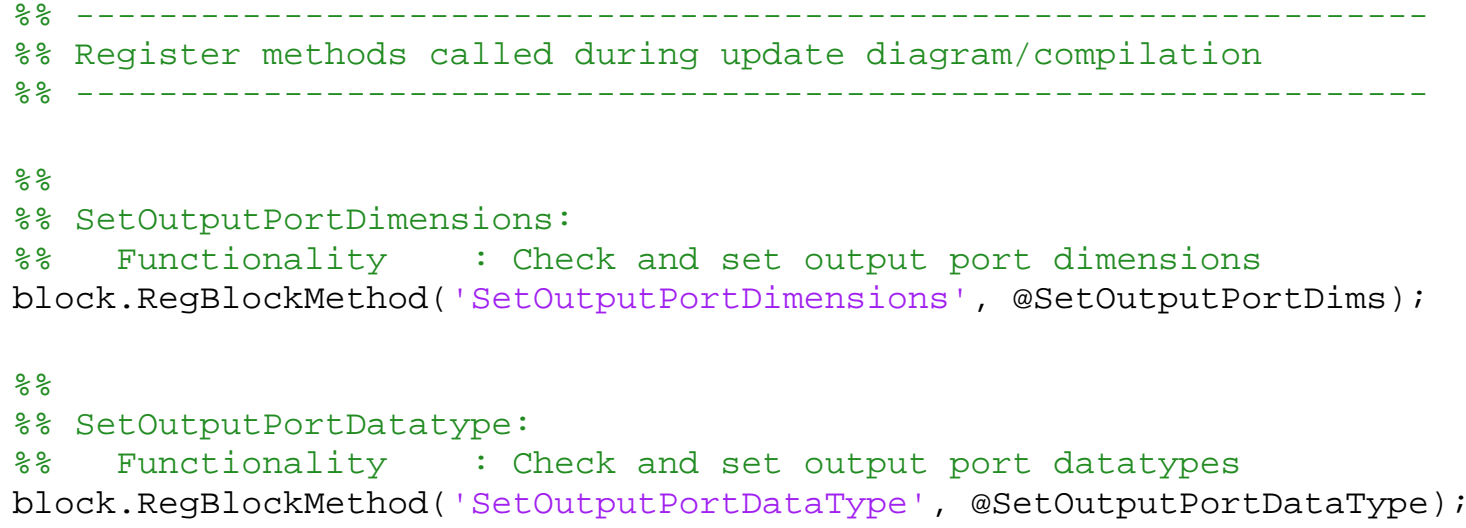




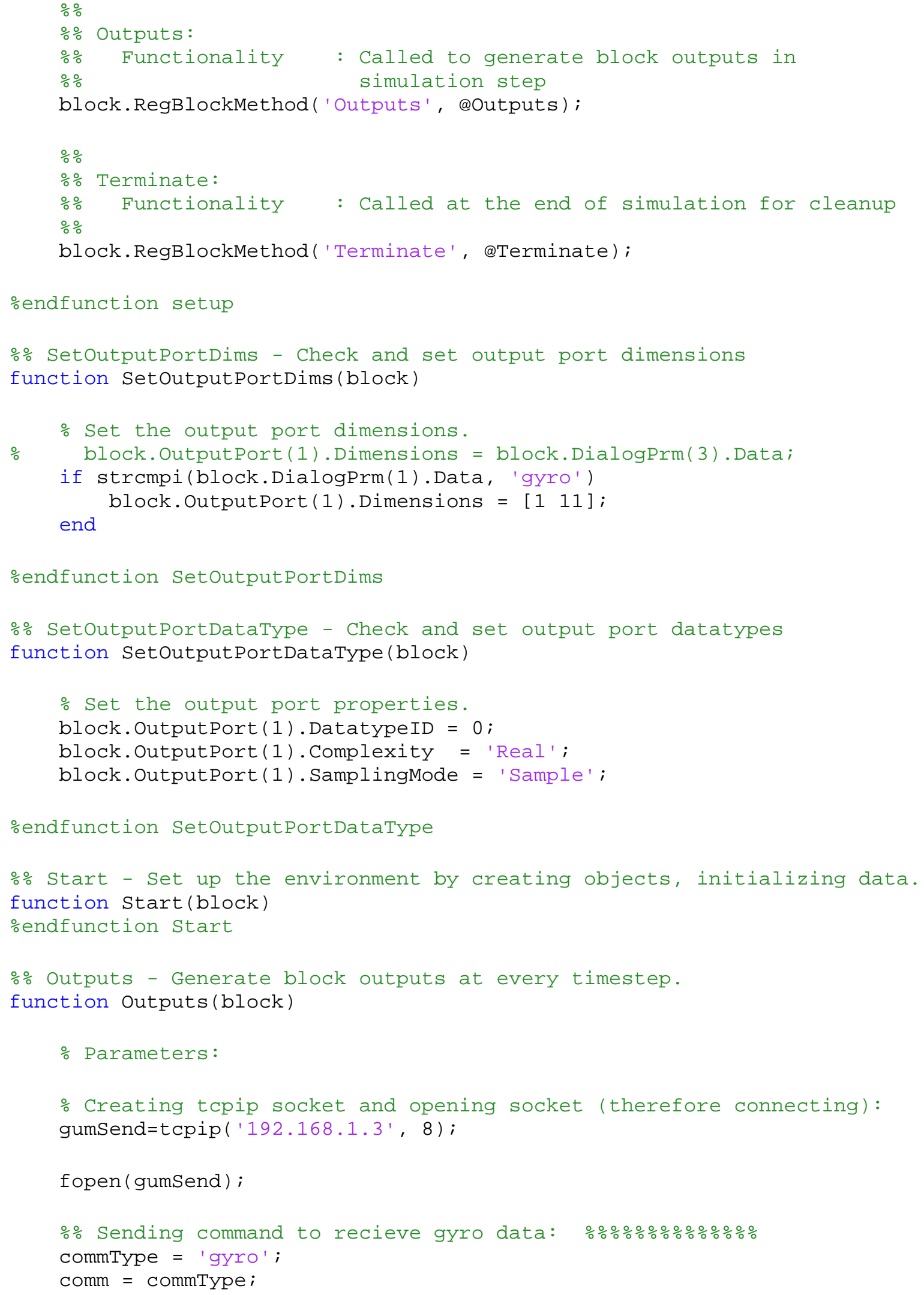




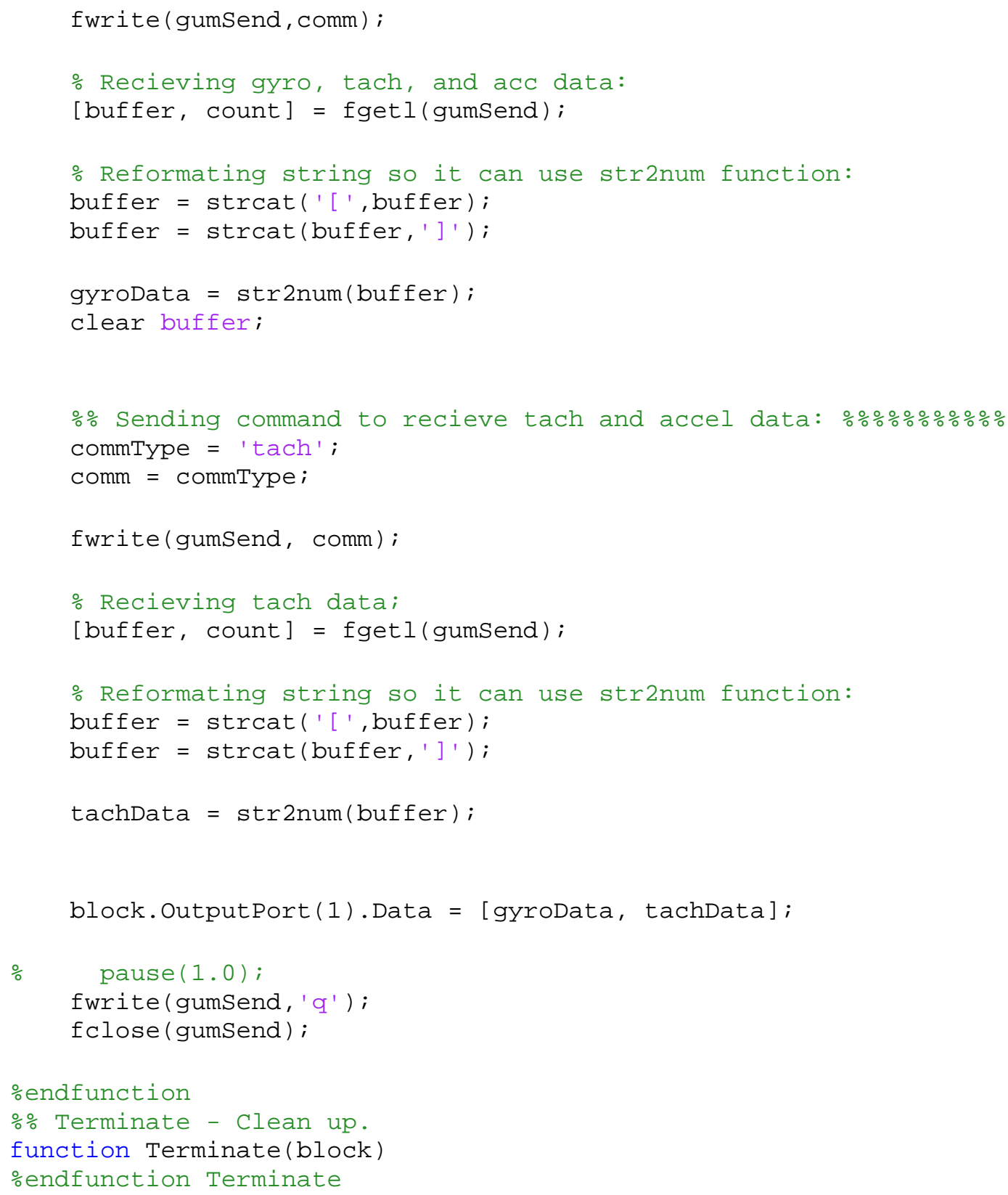

\section{sysIDStart.m}

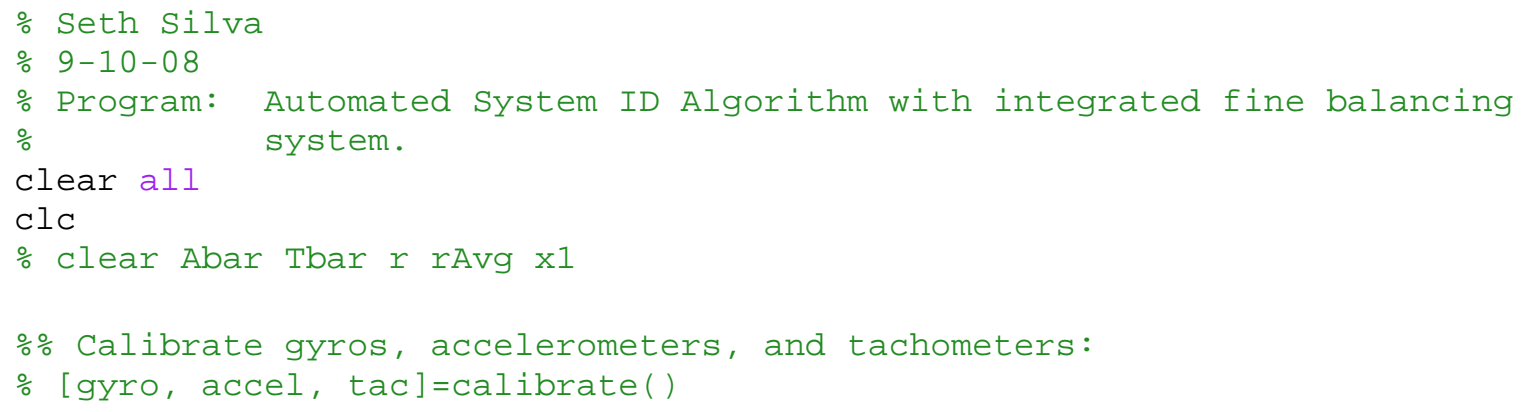




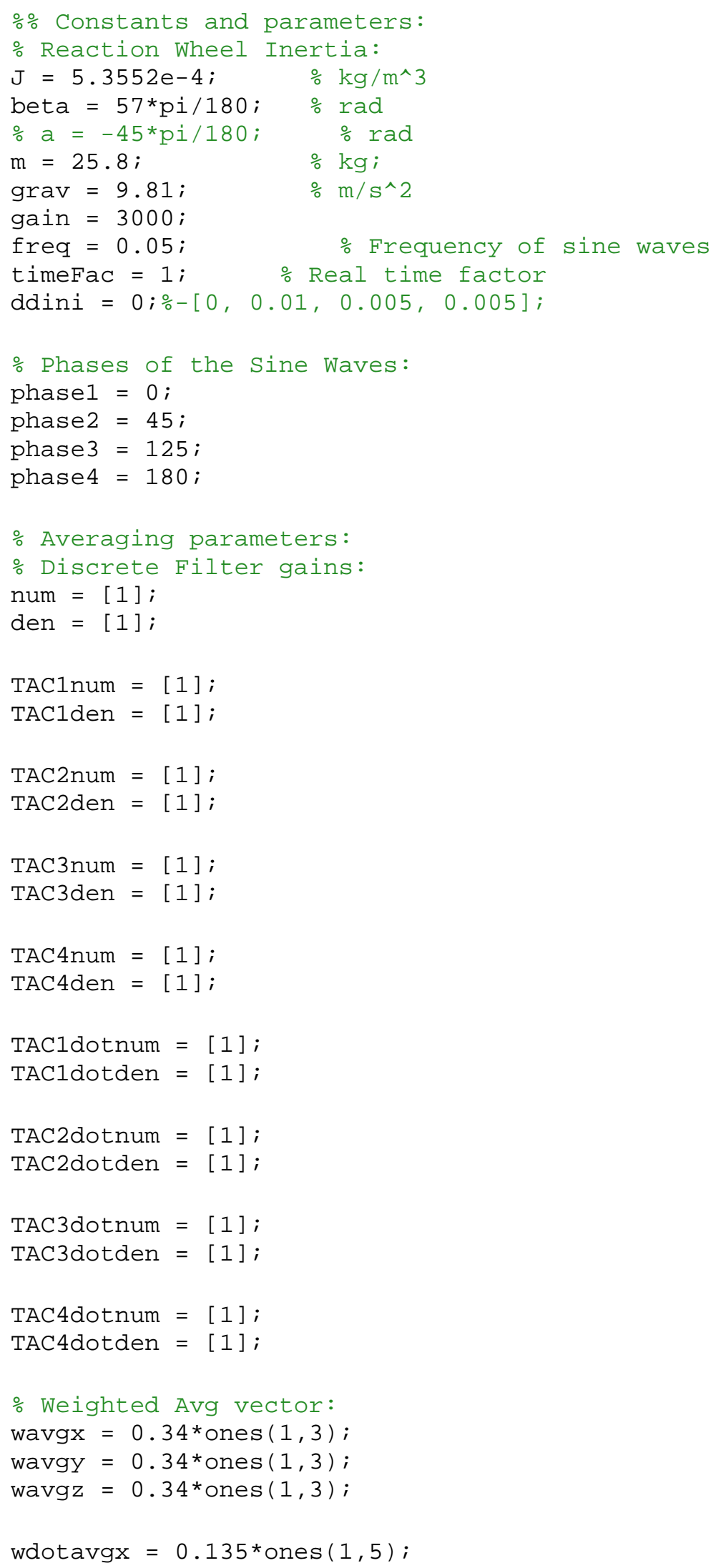




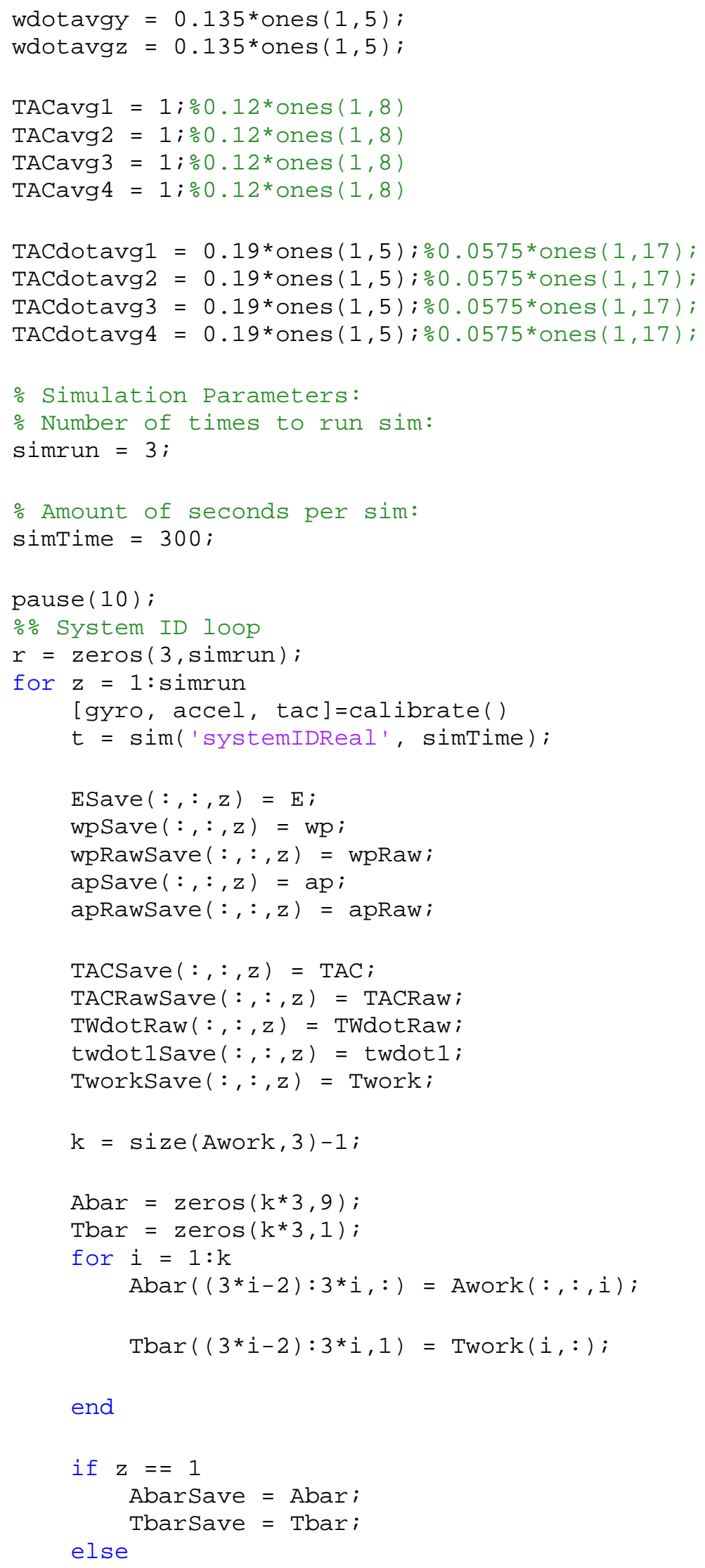




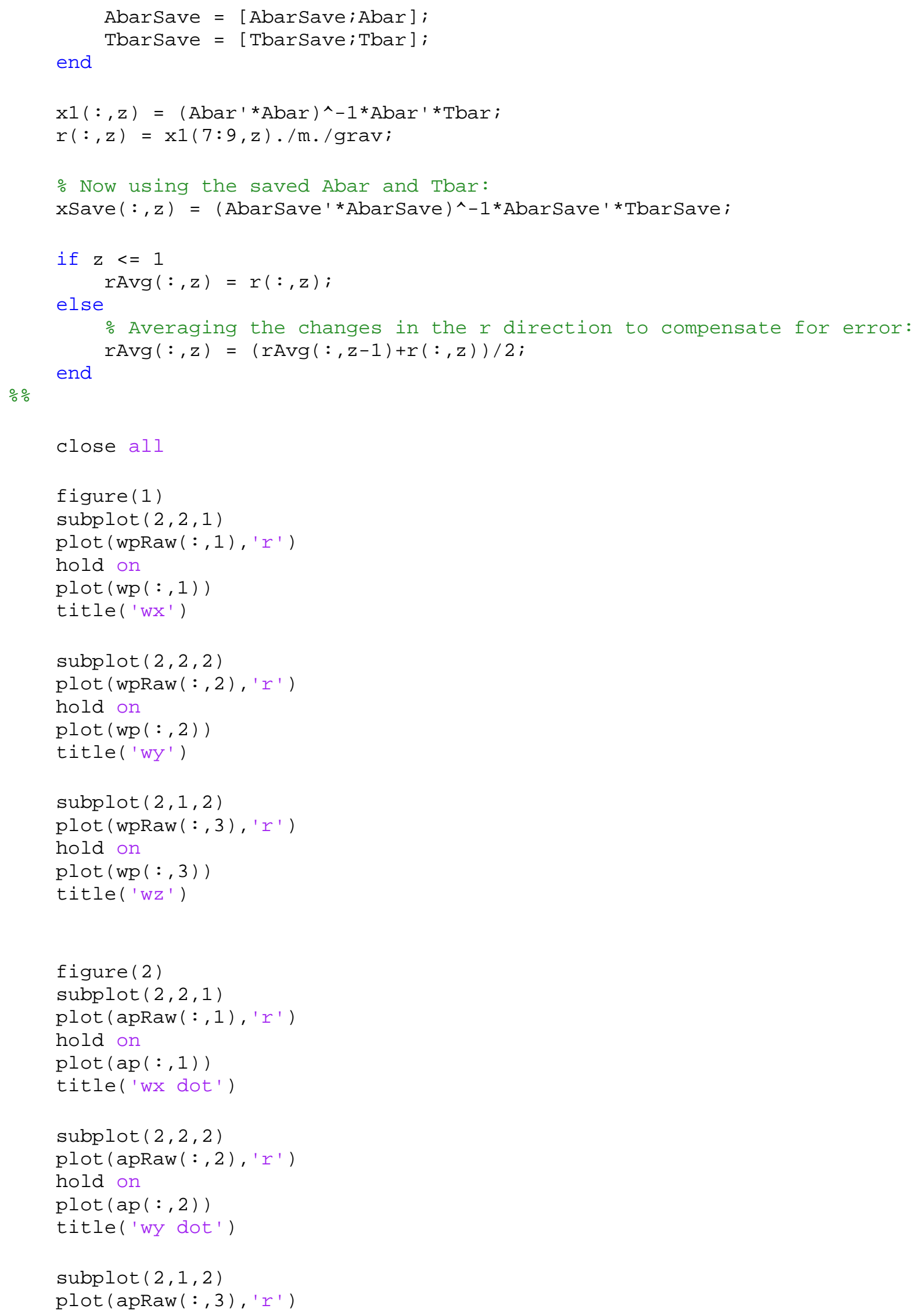




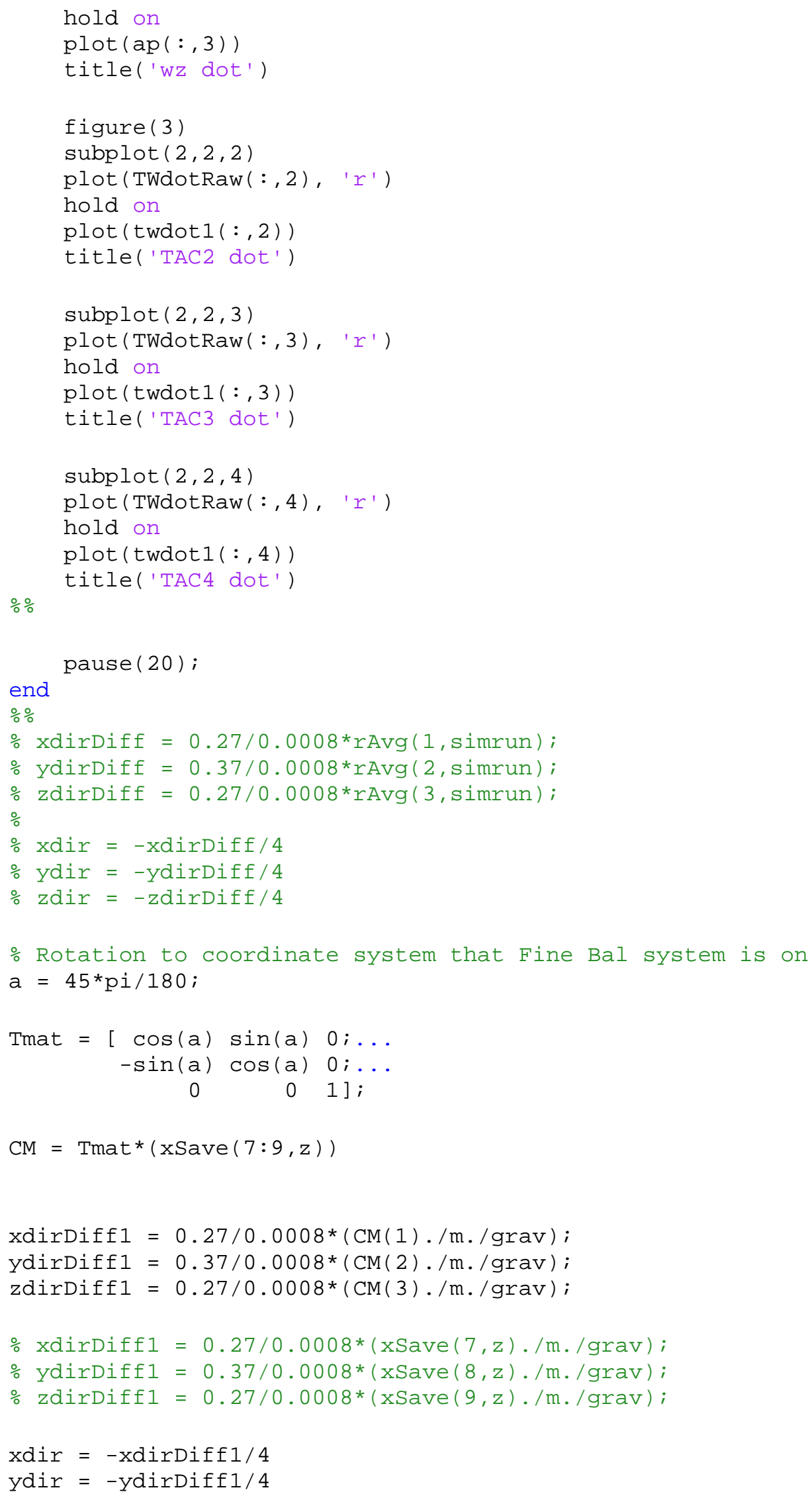




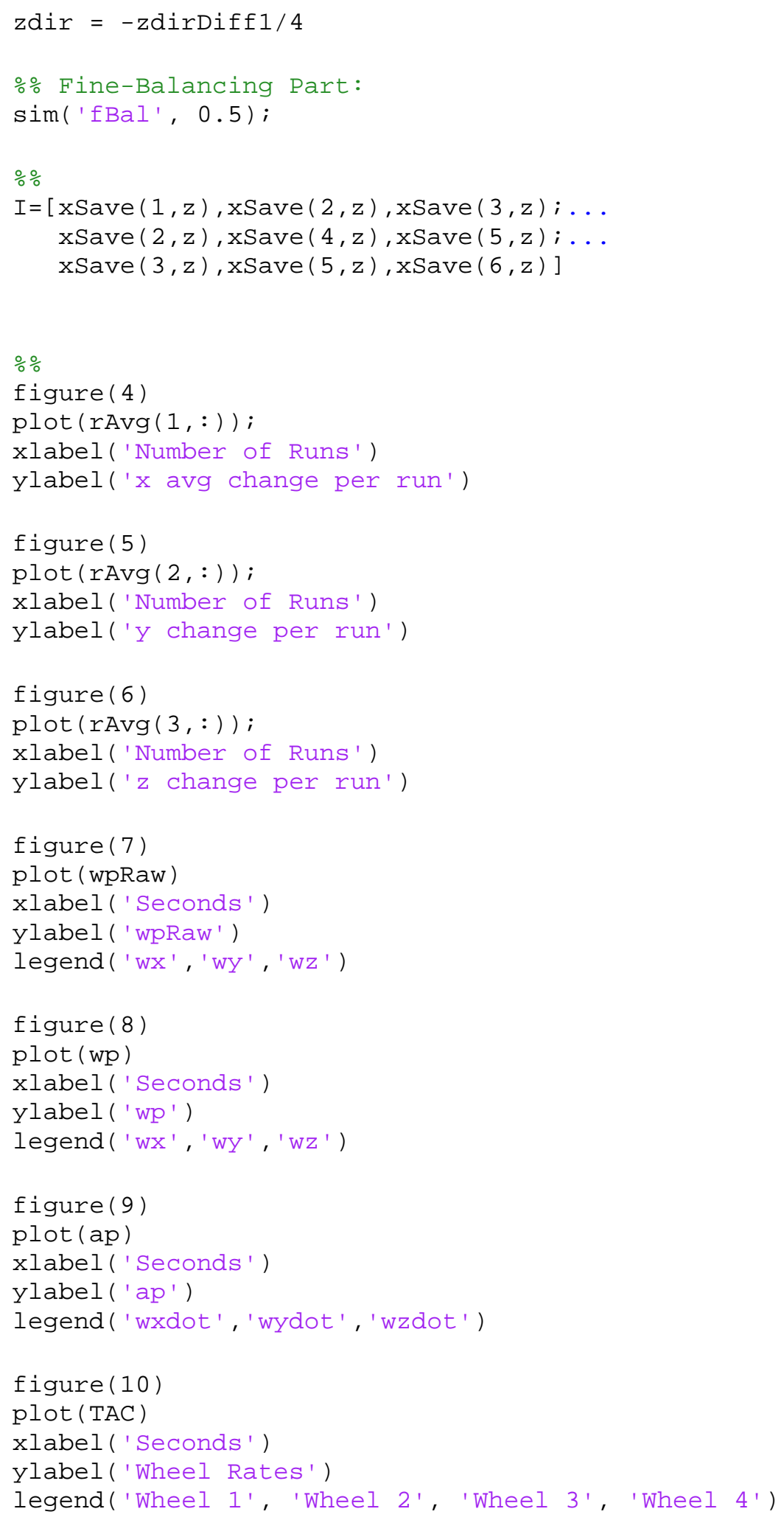


systemIDReal.mdl simulation

Full model:

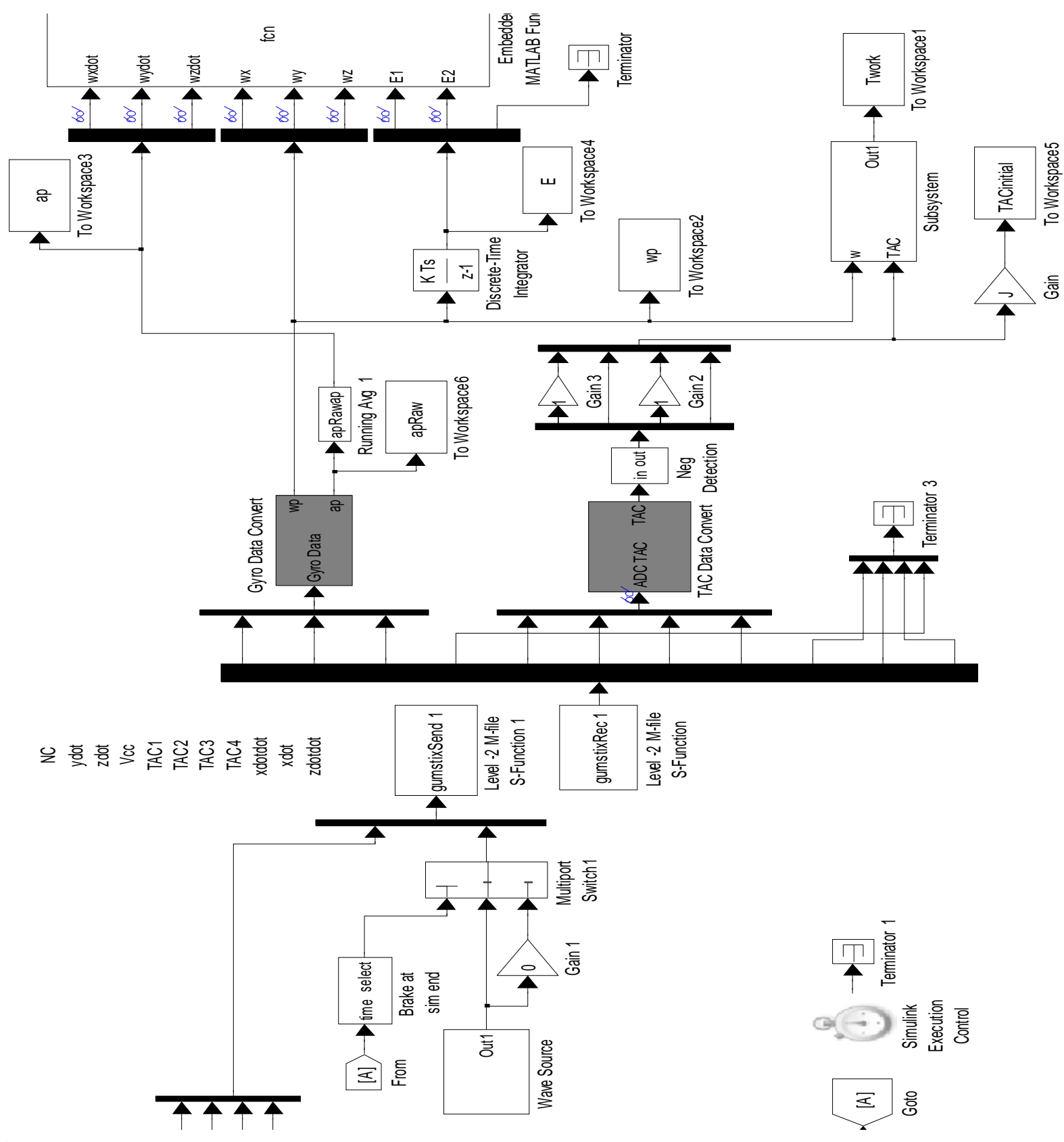

Wave Source: 


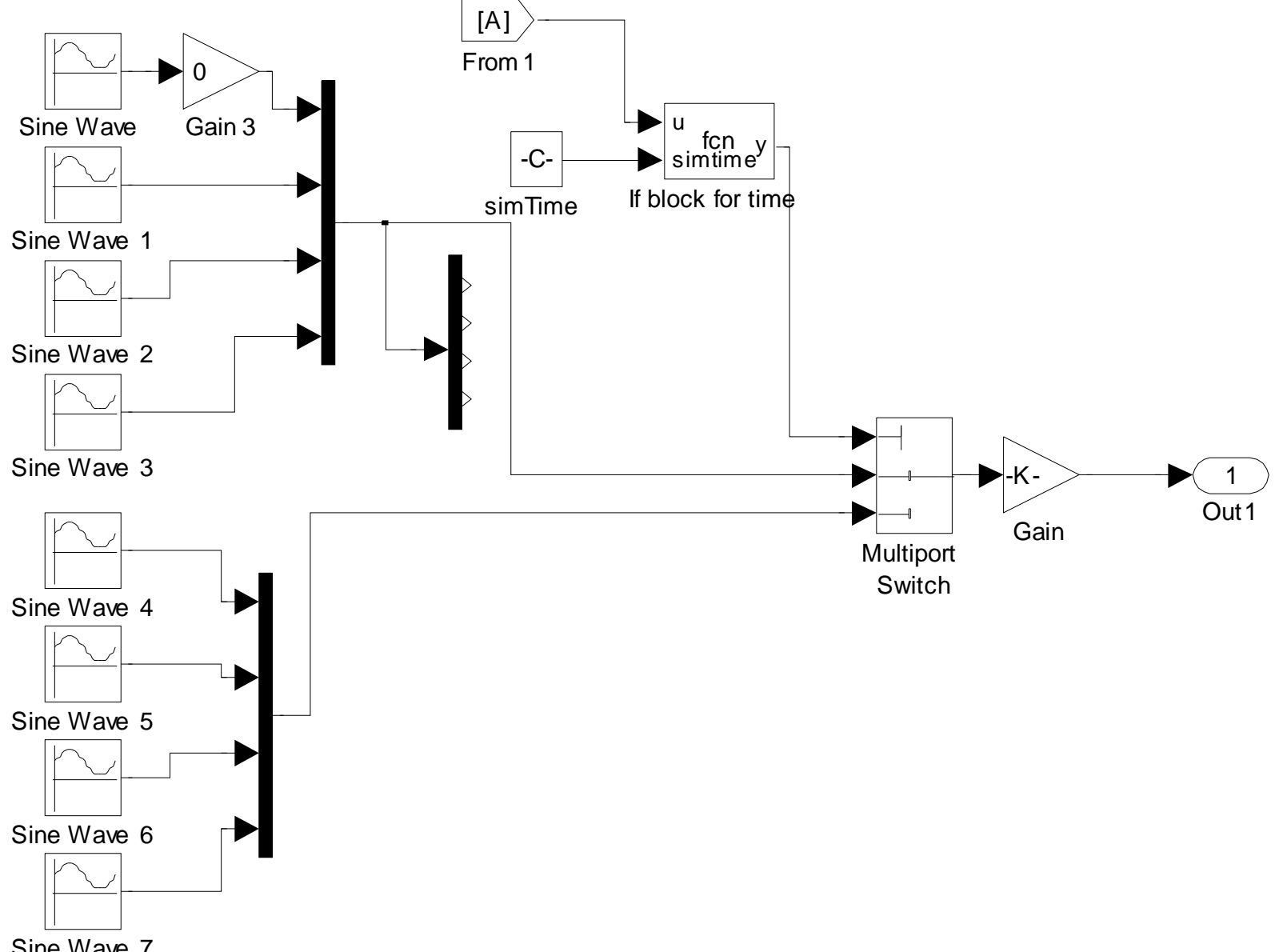

Brake at sim end:

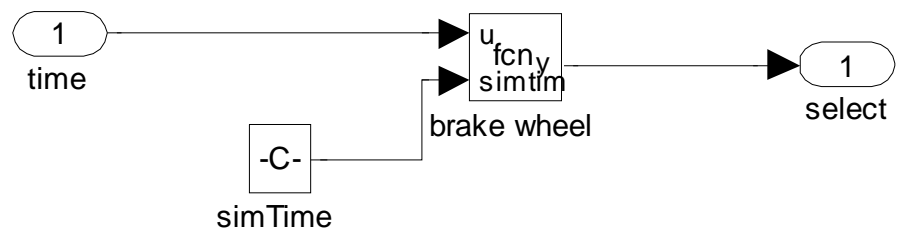

Gyro Data Convert: 


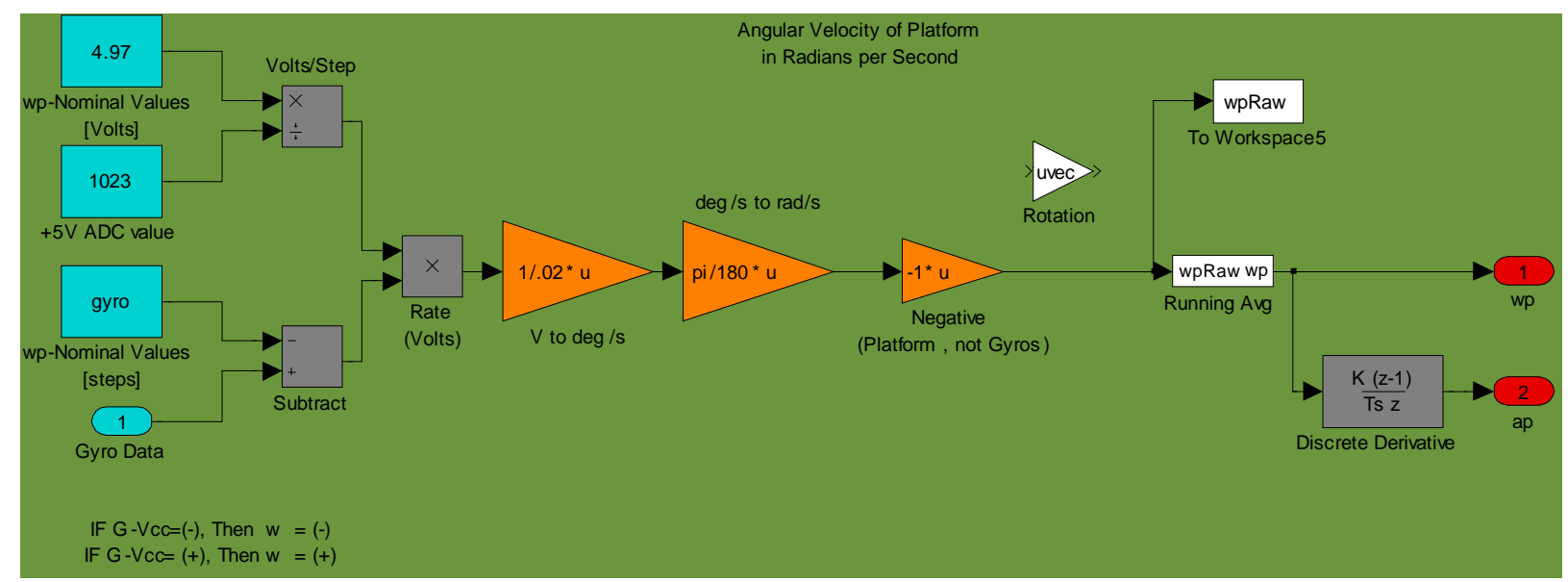

\section{TAC Data Convert:}

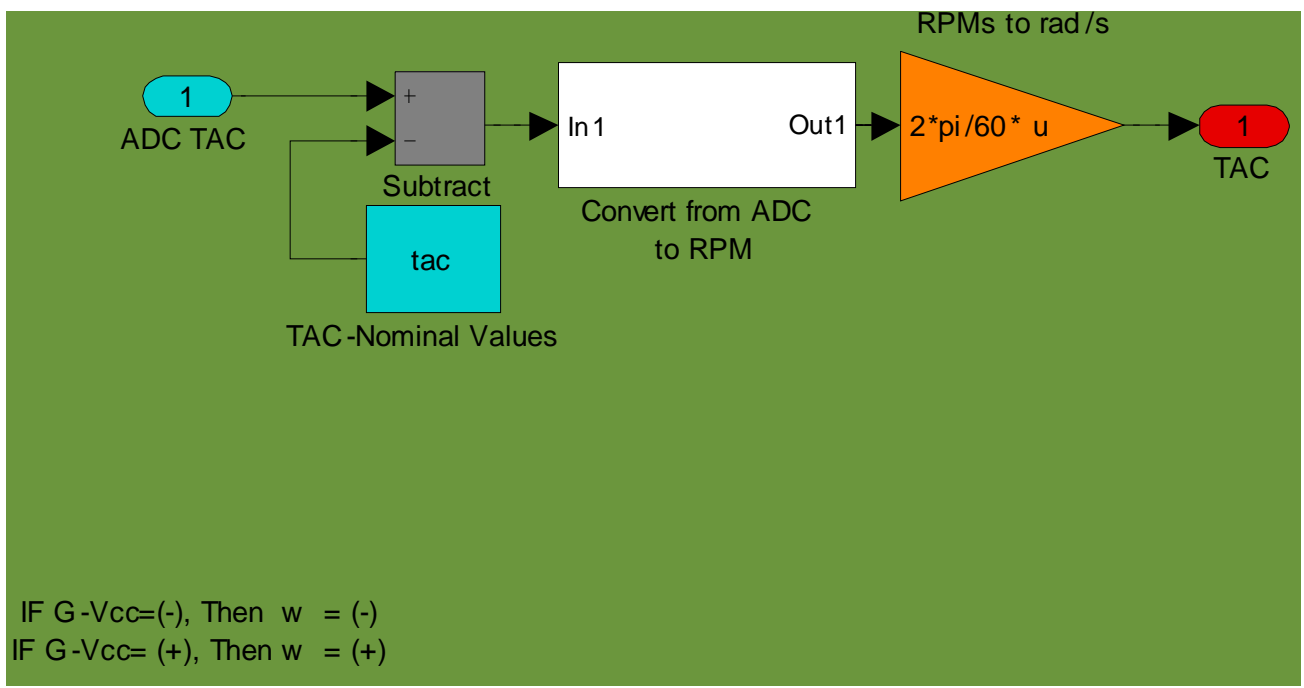

Running Avg:

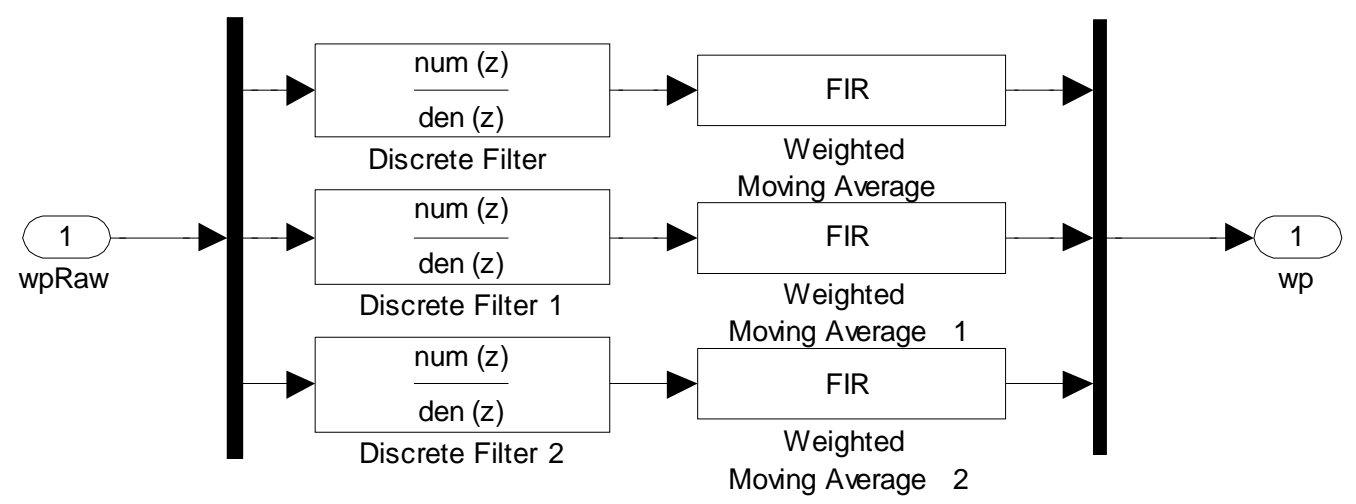

Running Avg1: 


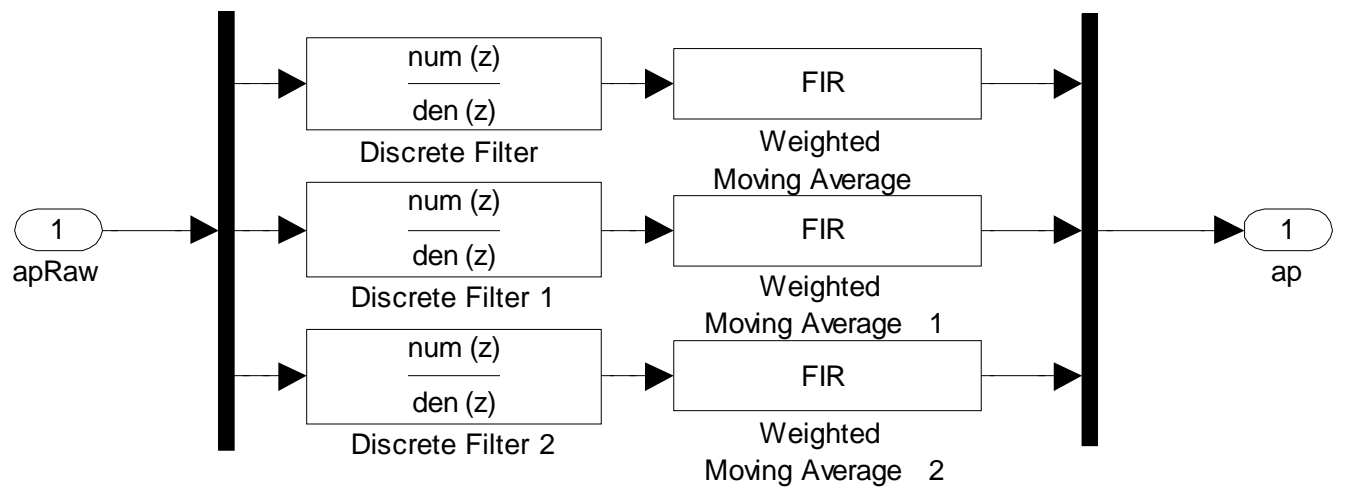

Neg Detection:

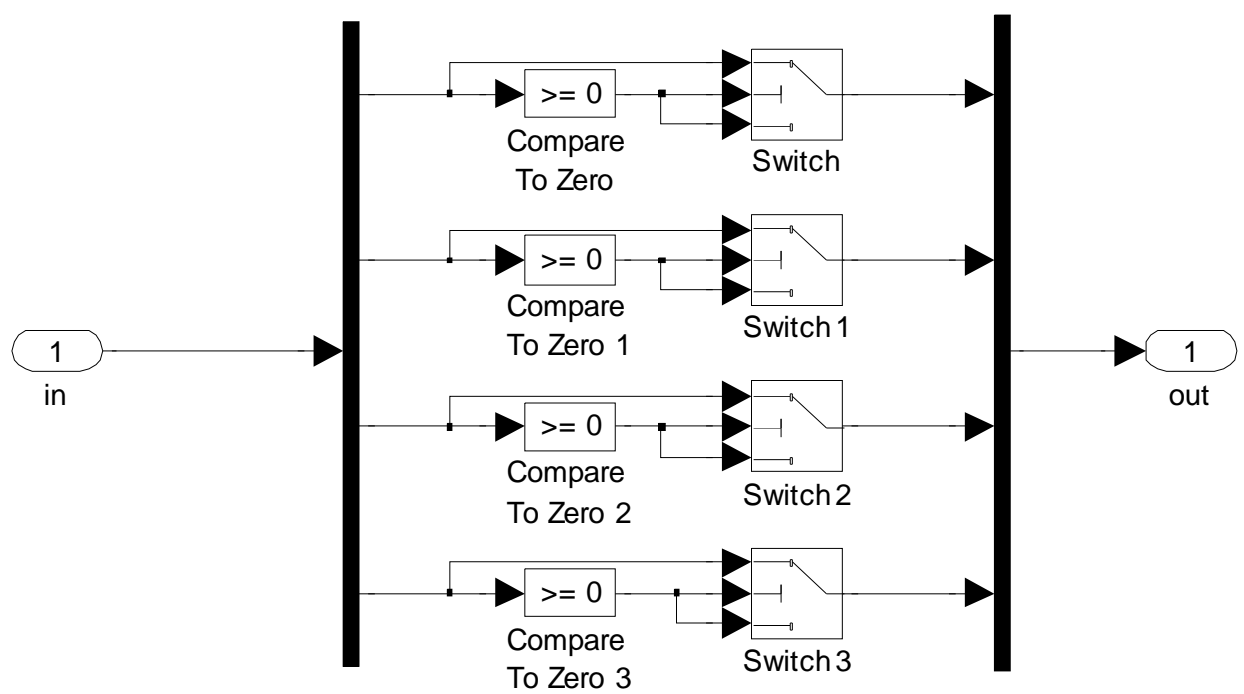

Torque:

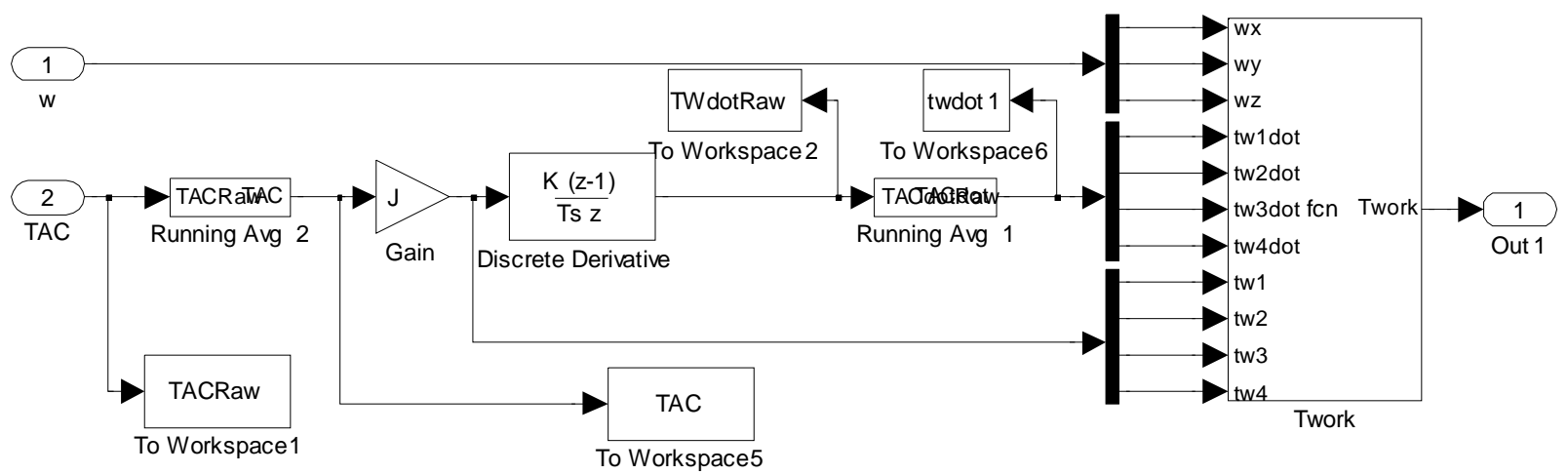

Running Avg2: 


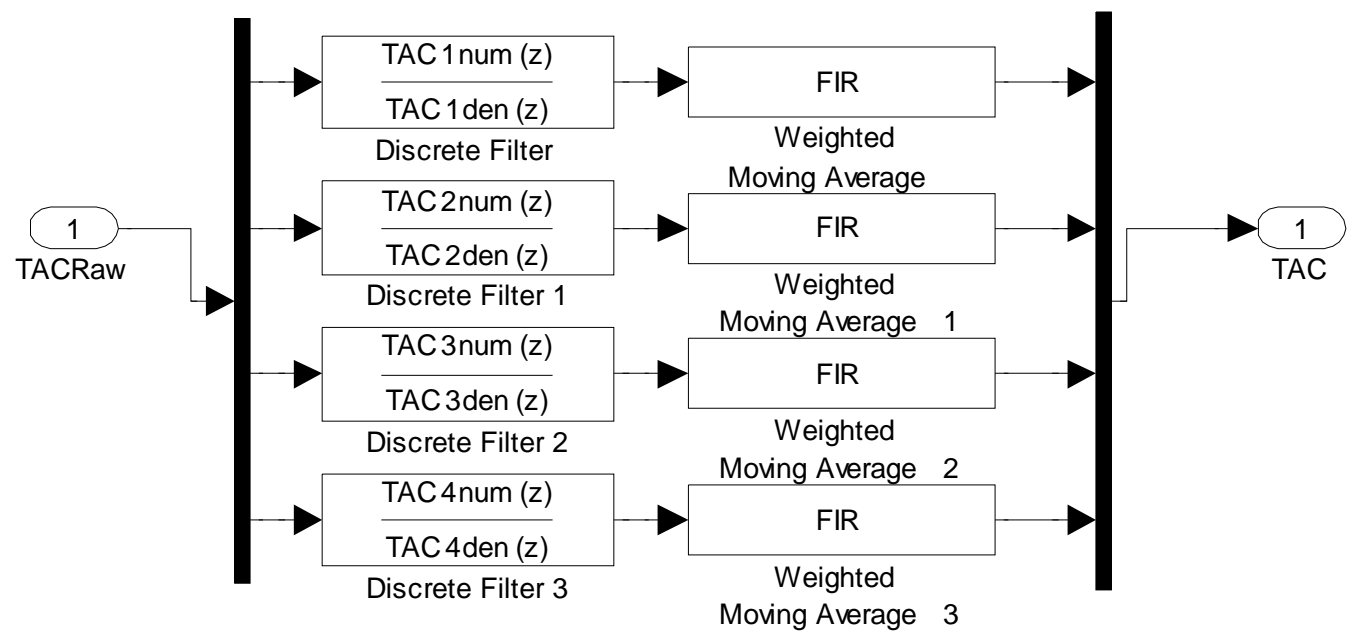

Running Avg3:

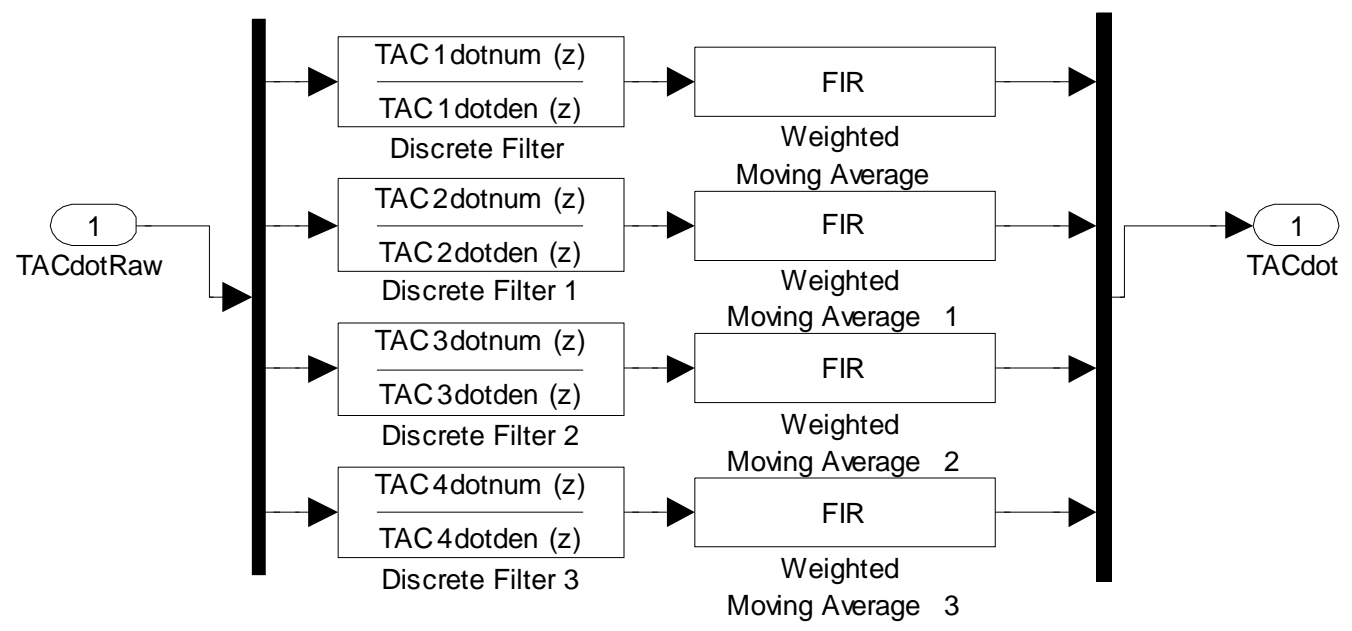

MATLAB embedded function: A:

function $A=f c n$ (wxdot, wydot, wzdot, wx, wy, wz, E1, E2)

\% This block supports an embeddable subset of the MATLAB language.

ㅇ see the help menu for details.

- Compile A matrix for System ID

$A=\left[w x d o t\left(w y d o t-w x^{\star} w z\right)\left(w z \operatorname{dot}+w x^{\star} w y\right)-w^{*} w z \quad\left(w Y^{\wedge} 2-w z^{\wedge} 2\right) \quad w y^{\star} w z \quad 0 \quad-\right.$ $\cos (E 1){ }^{*} \cos (E 2) \sin (E 1){ }^{*} \cos (E 2)$

$\mathrm{wx}^{\star} \mathrm{wz} \quad\left(\mathrm{wxdot}+\mathrm{wy}^{\star} \mathrm{wz}\right) \quad\left(\mathrm{wz}^{\wedge} 2-\mathrm{wx}^{\wedge} 2\right) \quad$ wydot $\left(\mathrm{wzdot}-\mathrm{wx}{ }^{\star} \mathrm{wy}\right)-\mathrm{wx}^{\star} \mathrm{wz}$ $\cos (\mathrm{E} 1){ }^{*} \cos (\mathrm{E} 2) \quad 0 \sin (\mathrm{E} 2)$

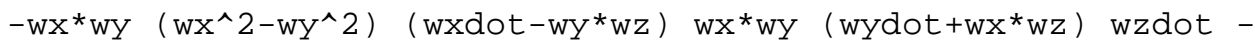
$\sin (\mathrm{E} 1) * \cos (\mathrm{E} 2)-\sin (\mathrm{E} 2) 0]$;

MATLAB embedded function: Twork:

function Twork $=f \mathrm{cn}(\mathrm{wx}, \mathrm{wy}, \mathrm{wz}, \mathrm{tw1}$ dot, $t w 2$ dot, $t w 3$ dot, $t w 4 d o t, t w 1, t w 2$, tw3, tw4)

\% This block supports the Embedded MATLAB subset.

\% See the help menu for details. 


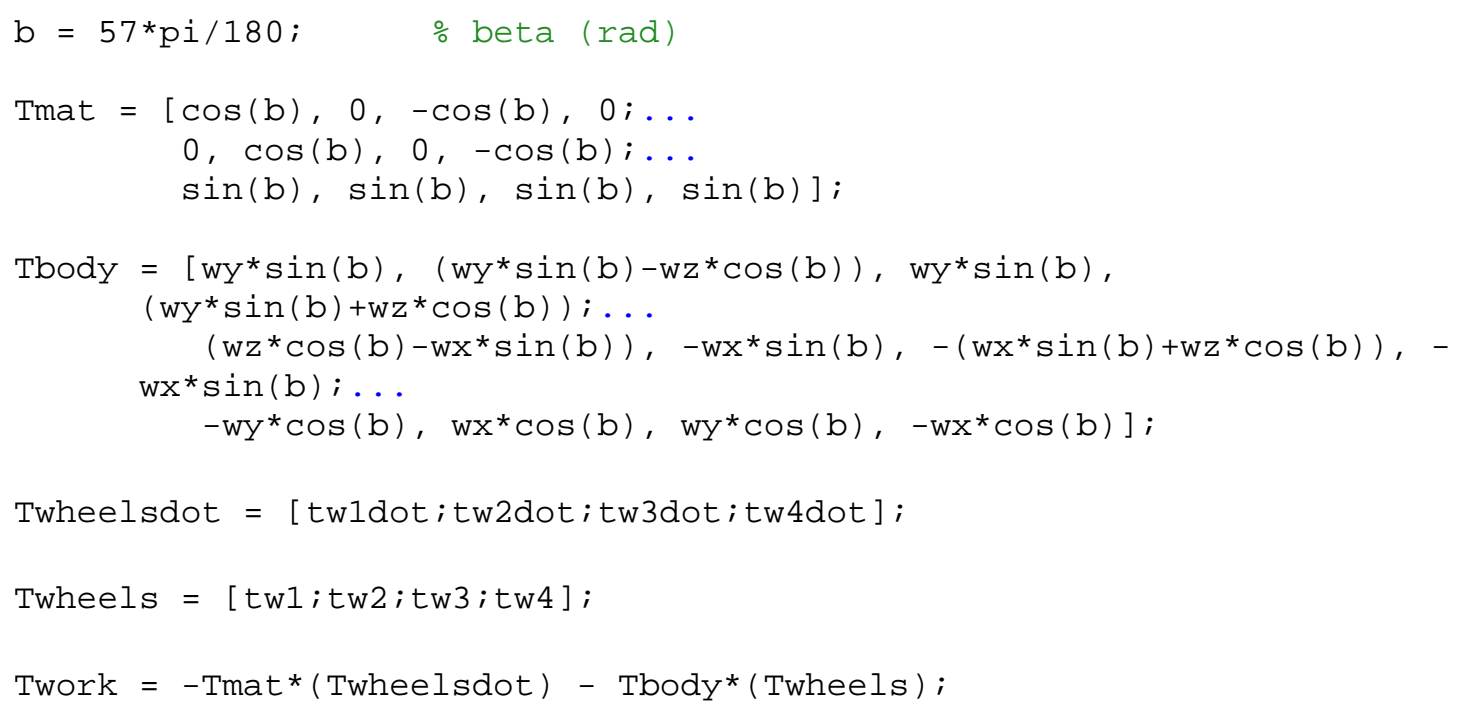




\section{Appendix C: MATLAB Code and SIMULINK Models for Post-processing}

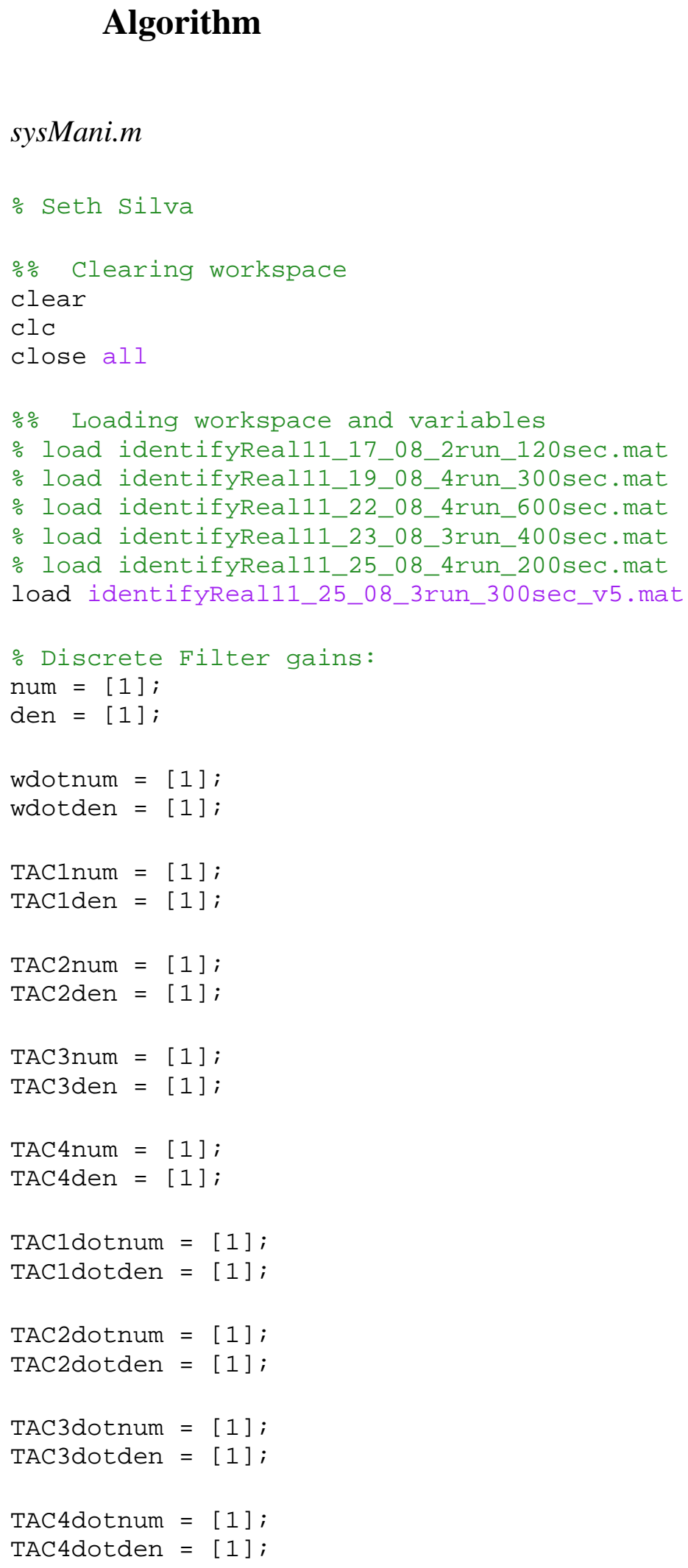




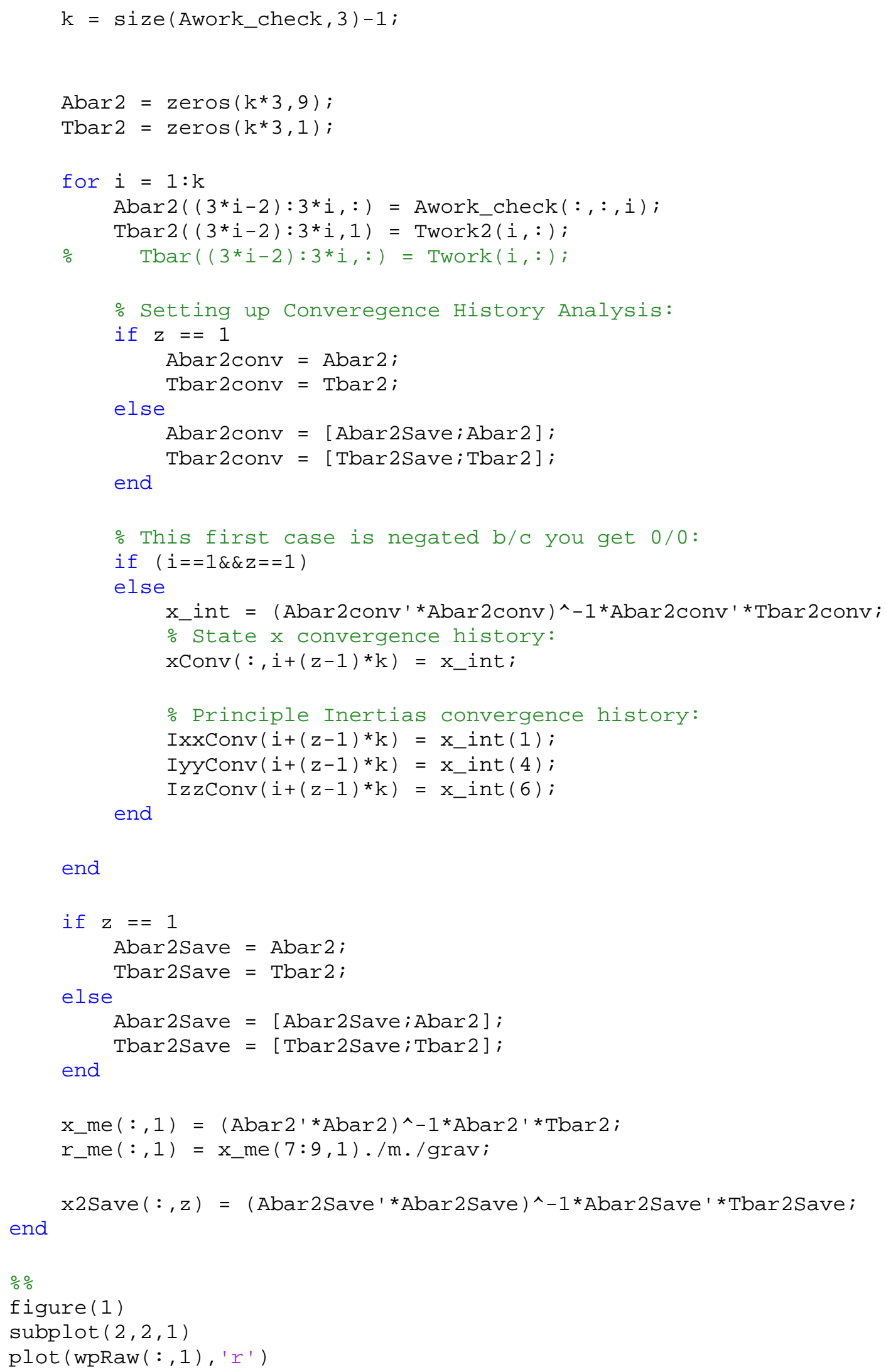




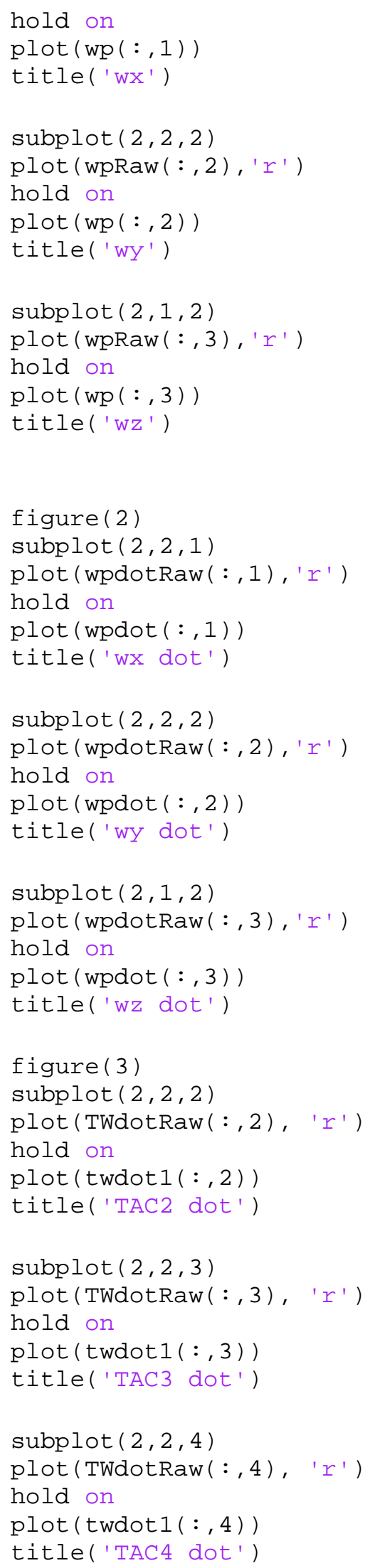


$\left(x 1(1, z)-x \_m e(1)\right) / x \_m e(1)$
$\left(x 1(4, z)-x \_m e(4)\right) / x \_m e(4)$
$\left(x 1(6, z)-x \_m e(6)\right) / x \_m e(6)$

$x=[0.6499 ; 0.000458 ;-0.000540 ; 0.6001 ; 0.0015 ; 0.5598 ; 0.0405 ;-0.0981 ; 1.3878] ;$

Diff $=\left(x \_\right.$_int $\left.-x\right)$

PerDiff $=$ Diff./x_int

$\%$ Convergence History:

figure (4)

subplot $(2,1,1)$

plot ( IxxConv)

xlabel ('Seconds' )

ylabel ('Ixx ( $\left.\mathrm{kg} / \mathrm{m}^{\wedge} 2\right)$ ')

title('Ixx Convergence')

subplot $(2,1,2)$

plot ( IxxConv)

axis ([ [ $\left.\left.\begin{array}{llll}0 & 20 & -15 & 2\end{array}\right]\right)$

xlabel ('Seconds' )

ylabel ('Ixx $\left.\left.\left(\mathrm{kg} / \mathrm{m}^{\wedge} 2\right)\right)^{\prime}\right)$

title('Ixx Convergence magnified')

figure (5)

subplot $(2,1,1)$

plot (IyyConv)

xlabel ('Seconds')

ylabel ('Iyy ( $\left.\mathrm{kg} / \mathrm{m}^{\wedge} 2\right)$ ')

title ('Iyy Convergence')

subplot $(2,1,2)$

plot (IyyConv)

$\operatorname{axis}\left(\left[\begin{array}{llll}0 & 300 & -6 & 1\end{array}\right]\right)$

xlabel ('Seconds')

ylabel ('Iyy $\left(\mathrm{kg} / \mathrm{m}^{\wedge} 2\right)$ ')

title('Iyy Convergence magnified')

figure (6)

subplot $(2,1,1)$

plot (IzzConv)

xlabel ('Seconds')

ylabel ('Izz ( $\left.\mathrm{kg} / \mathrm{m}^{\wedge} 2\right)^{\prime}$ ')

title('Izz Convergence')

subplot $(2,1,2)$

plot (IzzConv)

$\operatorname{axis}\left(\left[\begin{array}{llll}0 & 300 & -2 & 2\end{array}\right]\right)$

xlabel ('Seconds')

ylabel ('Izz ( $\left.\mathrm{kg} / \mathrm{m}^{\wedge} 2\right)$ ')

title('Izz Convergence magnified')

figure (7)

plot (wpRaw) 
xlabel ('Seconds')

ylabel ('Angular Velocity rad/sec')

title('Raw Angular Velocity') 
Full Model:

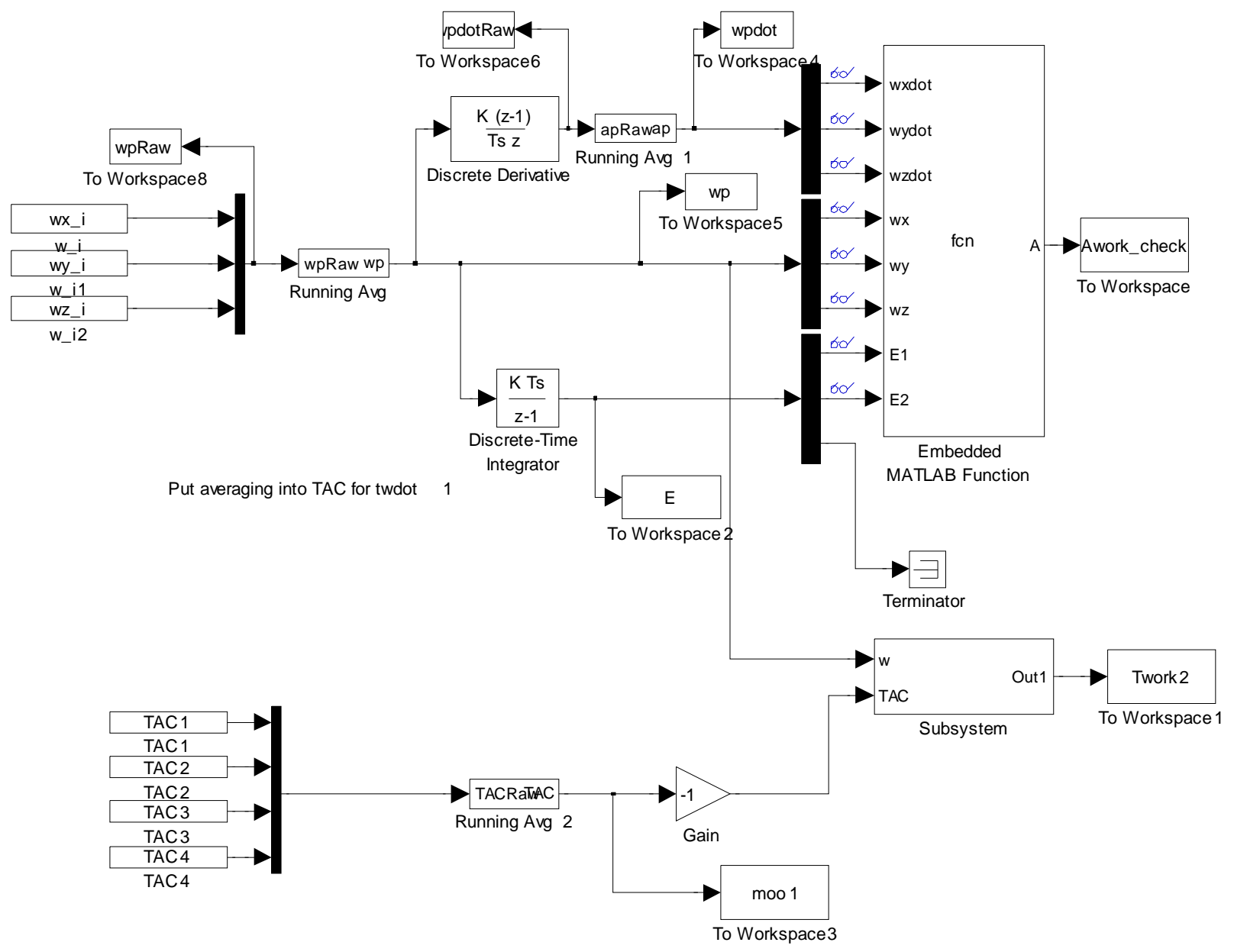

Running Avg:

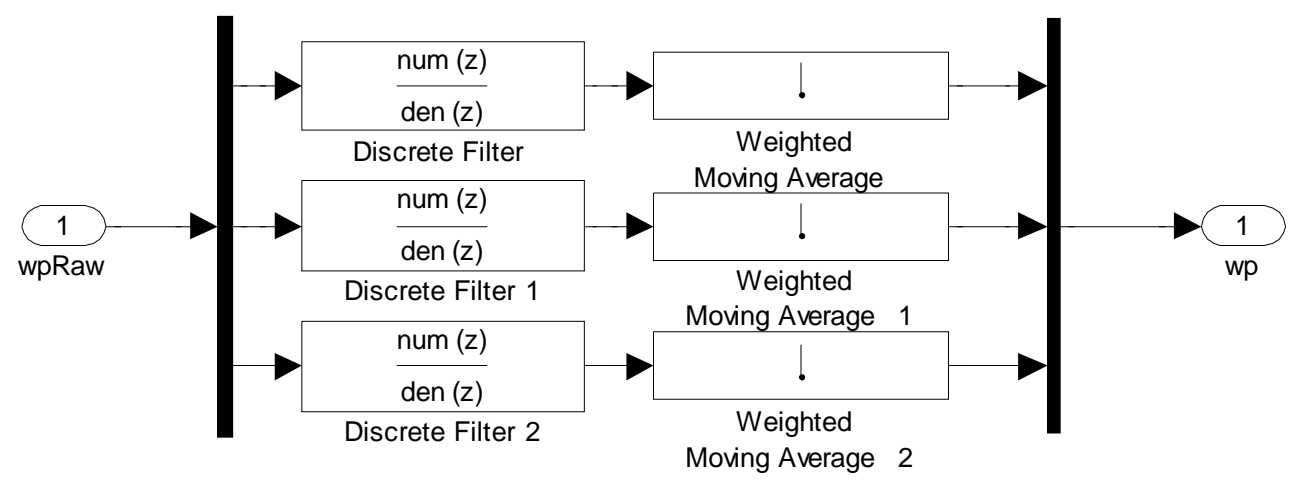


Running Avg1:

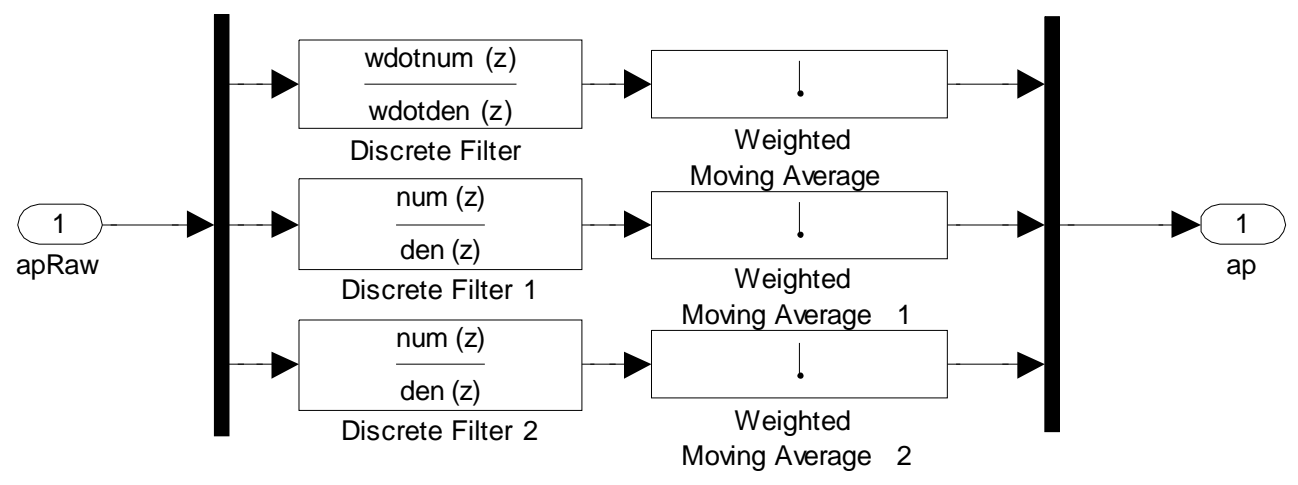

Running Avg2:

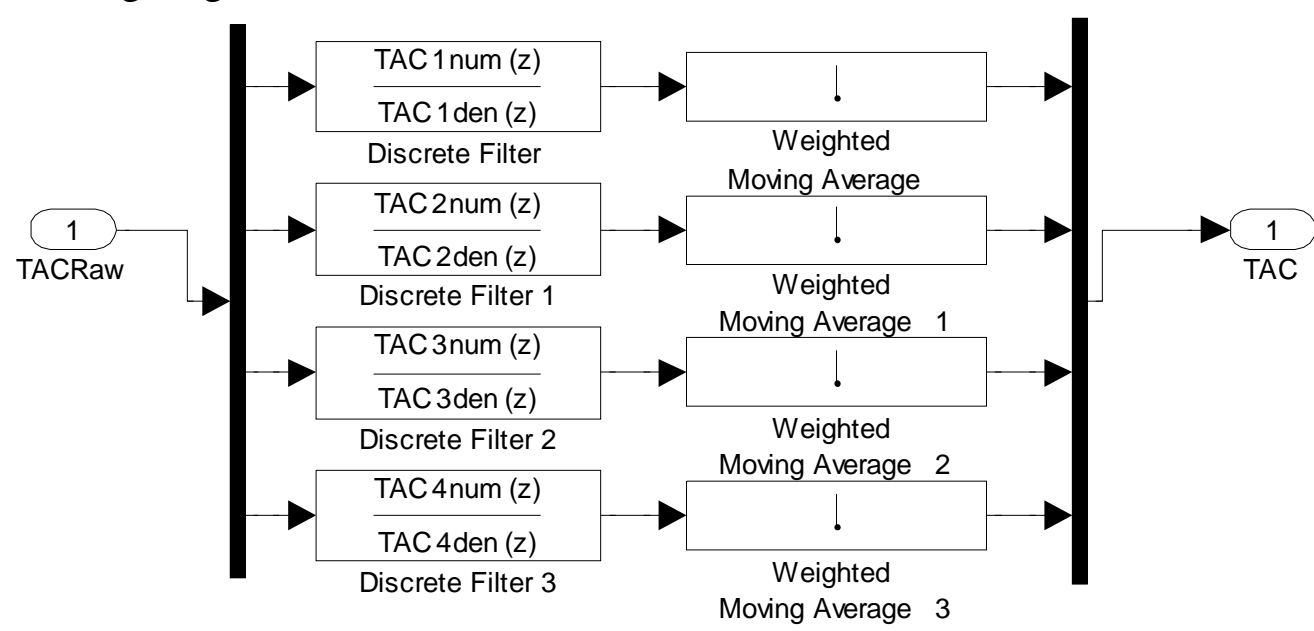

MATLAB embedded function: A:

function $A=f c n$ (wxdot, wydot, wzdot, wx, wy, wz, E1, E2)

ㅇ This block supports an embeddable subset of the MATLAB language.

o see the help menu for details.

을 Compile A matrix for system ID

$A=\left[w x d o t \quad\left(w y d o t-w x^{\star} w z\right) \quad\left(w z d o t+w x^{\star} w y\right)-w y^{\star} w z \quad\left(w y^{\wedge} 2-w z^{\wedge} 2\right) \quad w y^{\star} w z \quad 0-\right.$ $\cos (E 1){ }^{*} \cos (E 2) \sin (E 1){ }^{\star} \cos (E 2)$

$\mathrm{wx}^{\star} \mathrm{wz}^{\star} \quad\left(\mathrm{wxdot}+\mathrm{wy}^{\star} \mathrm{wz}\right) \quad\left(\mathrm{wz}^{\wedge} 2-\mathrm{wx}^{\wedge} 2\right)$ wydot (wzdot $\left.-\mathrm{wx}^{\star} \mathrm{wy}\right)-\mathrm{wx}^{\star} \mathrm{wz}$

$\cos (\mathrm{E} 1){ }^{*} \cos (\mathrm{E} 2) \quad 0 \quad \sin (\mathrm{E} 2)$

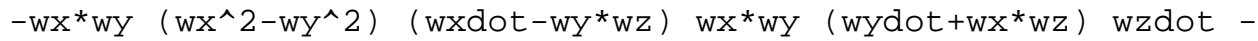

$\sin (\mathrm{E} 1) * \cos (\mathrm{E} 2)-\sin (\mathrm{E} 2) 0]$; 
Torque:

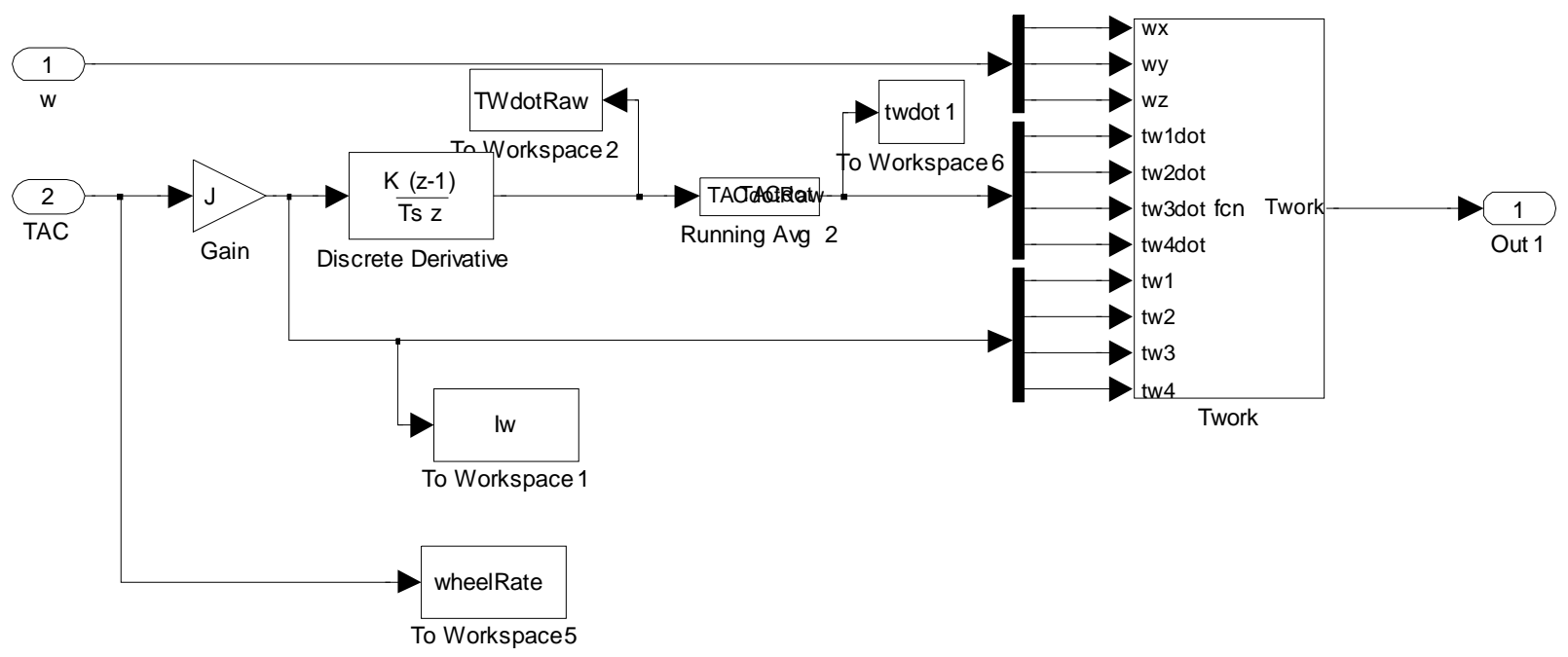

Running Avg3:

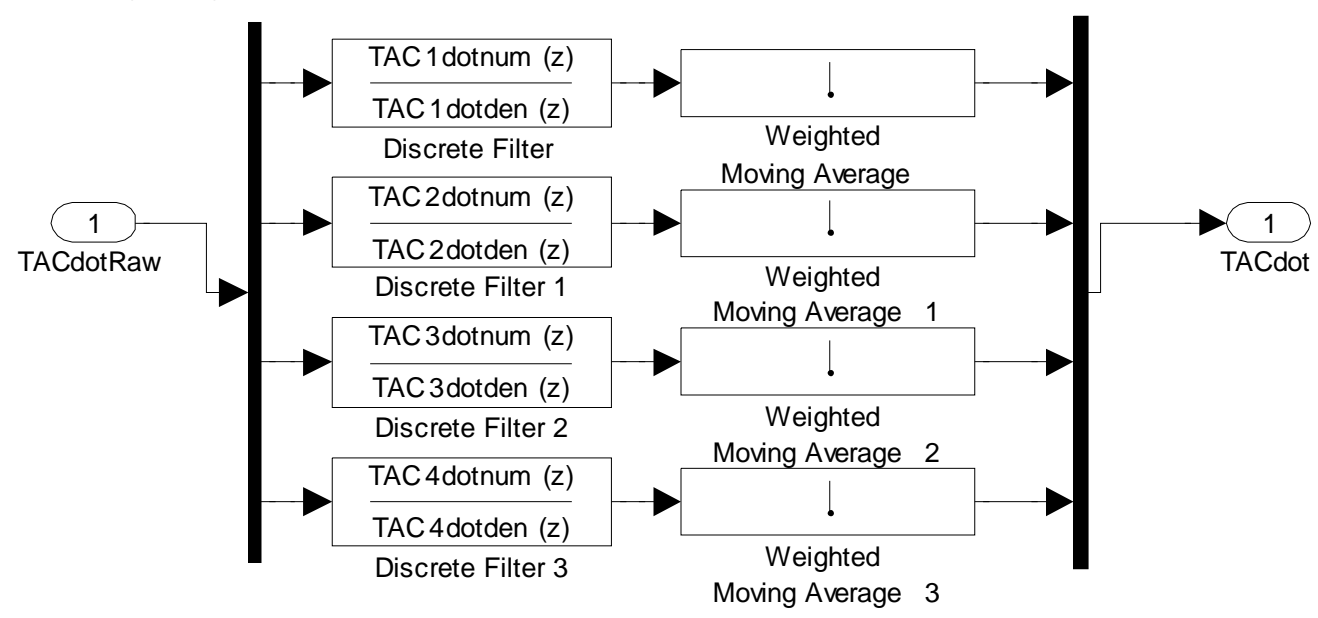

MATLAB embedded function: Twork:

function Twork $=f \mathrm{cn}(w x, w y, w z, t w 1 d o t, t w 2 d o t, t w 3 d o t, t w 4 d o t, t w 1, t w 2$, tw3, tw4)

This block supports the Embedded MATLAB subset.

o see the help menu for details.

을 Distribute wheel torques to 3 axes

$\mathrm{Tw} 1=\mathrm{tw} 1$ dot;

$\mathrm{Tw} 2=\mathrm{tw} 2 \operatorname{dot} ;$

Tw3 = tw3dot;

$\mathrm{Tw} 4=\mathrm{tw} 4 \operatorname{dot}$;

$\mathrm{IW} 1=\mathrm{tw} 1$;

$\mathrm{Iw} 2=t \mathrm{w} 2$;

Iw3 = tw 3 ;

$\operatorname{Iw} 4=\mathrm{tw} 4 ;$

$\mathrm{b}=57{ }^{*} \mathrm{pi} / 180$; 


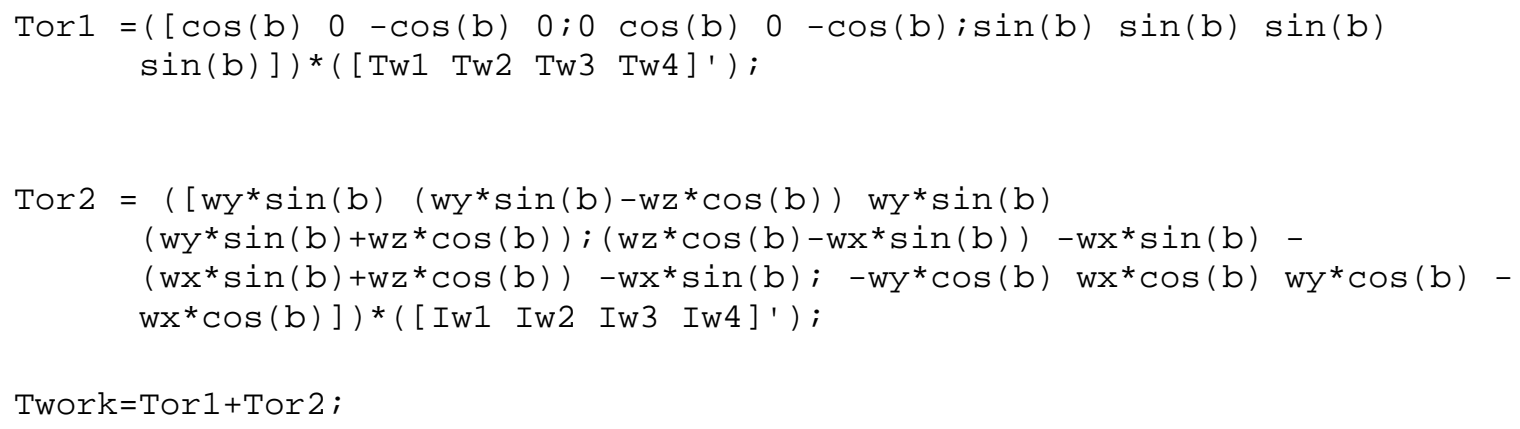

